MAGNETIC RESONANCE CURRENT DENSITY IMAGING USING ONE COMPONENT OF MAGNETIC FLUX DENSITY

A THESIS SUBMITTED TO

THE GRADUATE SCHOOL OF NATURAL AND APPLIED SCIENCES OF

MIDDLE EAST TECHNICAL UNIVERSITY

BY

ALİ ERSÖZ

IN PARTIAL FULFILLMENT OF THE REQUIREMENTS

FOR

THE DEGREE OF MASTER OF SCIENCE

IN

ELECTRICAL AND ELECTRONICS ENGINEERING

JULY 2010 
Approval of the Thesis:

\section{MAGNETIC RESONANCE CURRENT DENSITY IMAGING USING ONE COMPONENT OF MAGNETIC FLUX DENSITY}

submitted by ALI ERSÖZ in partial fulfillment of the requirements for the degree of Master of Science in Electrical and Electronics Engineering Department, Middle East Technical University by,

Prof. Dr. Canan Özgen

Dean, Graduate School of Natural and Applied Sciences

Prof. Dr. İsmet Erkmen

Head of Department, Electrical and Electronics Engineering

Prof. Dr. B. Murat Eyüboğlu

Supervisor, Electrical and Electronics Engineering Dept., METU

\section{Examining Committee Members:}

Prof. Dr. Nevzat Güneri Gençer

Electrical and Electronics Engineering Dept., METU

Prof. Dr. B. Murat Eyüboğlu

Electrical and Electronics Engineering Dept., METU

Assist. Prof. Dr. Yeşim Serinağaoğlu Doğrusöz

Electrical and Electronics Engineering Dept., METU

Prof. Dr. Yusuf Ziya İder

Electrical and Electronics Engineering Dept., Bilkent University

Prof. Dr. Adnan Köksal

Electrical and Electronics Engineering Dept., Hacettepe University

Date: 
I hereby declare that all information in this document has been obtained and presented in accordance with academic rules and ethical conduct. I also declare that, as required by these rules and conduct, I have fully cited and referenced all material and results that are not original to this work.

Name Last name: Ali ERSÖZ

Signature: 


\begin{abstract}
MAGNETIC RESONANCE CURRENT DENSITY IMAGING USING ONE COMPONENT OF MAGNETIC FLUX DENSITY
\end{abstract}

\author{
Ersöz, Ali \\ MSc., Department of Electrical and Electronics Engineering \\ Supervisor : Prof. Dr. B. Murat Eyüboğlu
}

July 2010, 96 pages

Magnetic Resonance Electrical Impedance Tomography (MREIT) algorithms using current density distribution have been proposed in the literature. The current density distribution, $\vec{J}$, can be determined by using Magnetic Resonance Current Density Imaging (MRCDI) technique. In MRCDI technique, all three components of magnetic flux density, $\vec{B}$, should be measured. Hence, object should be rotated inside the magnet which is not trivial even for small size objects and remains as a strong limitation to clinical applicability of the technique. In this thesis, 2D MRCDI problem is investigated in detail and an analytical relation is found between $B_{z}, J_{x}$ and $J_{y}$. This study makes it easy to understand the behavior of $B_{z}$ due to changes in $J_{x}$ and $J_{y}$. Furthermore, a novel 2D MRCDI reconstruction algorithm using one component of $\vec{B}$ is proposed. Iterative FT-MRCDI algorithm is also implemented. The algorithms are tested with simulation and experimental models. In simulations, error in the reconstructed current density changes between $0.27 \%-23.00 \%$ using the proposed algorithm and $7.41 \%-37.45 \%$ using the iterative FT-MRCDI algorithm for 
various SNR levels. The proposed algorithm is superior to the iterative FT-MRCDI algorithm in reconstruction time comparison. In experimental models, the classical MRCDI algorithm has the best reconstruction performance when the algorithms are compared by evaluating the reconstructed current density images perceptually. However, the J-substitution algorithm reconstructs the best conductivity image by using $\vec{J}$ obtained from the proposed algorithm. Finally, the iterative FT-MRCDI algorithm shows the best performance when the reconstructed current density images are verified by using divergence theorem.

Keywords: electrical impedance tomography, magnetic resonance imaging, current density imaging 


\title{
ÖZ
}

\section{TEK YÖNDEKİ MANYETİK AKI YOĞUNLUĞU KULLANARAK MANYETİK REZONANS AKIM YOĞUNLUĞU GÖRÜNTÜLEME}

\author{
Ersöz, Ali \\ Yüksek Lisans, Elektrik ve Elektronik Mühendisliği Bölümü \\ Tez yöneticisi : Prof. Dr. B. Murat Eyüboğlu
}

Temmuz 2010, 96 sayfa

Bir cismin elektriksel iletkenlik dağılımını hesaplamak için akım tabanlı Manyetik Rezonans Elektriksel Empedans Tomografi (MREIT) algoritmaları literatürde önerilmiştir. Akım yoğunluğu dağılımı, $\vec{J}$, Manyetik Rezonans Akım Yoğunluğu Görüntüleme (MRCDI) tekniği kullanılarak bulunabilir. MRCDI tekniğinde, manyetik ak1 yoğunluğunun, $\vec{B}$, üç yöndeki bileşkesinin de ölçülmesi gerekmektedir. Bu nedenle, cismin cihaz içinde döndürülmesi gerekmektedir. Cismin küçük olduğu durumlarda bile cismin cihaz içinde döndürülmesi kolay bir işlem değildir ve bu tekniğin klinik uygulamalarda kullanılmasını engelleyen en önemli etkenlerden biridir. $\mathrm{Bu}$ tez çalışmasında, 2 boyutlu MRCDI problem detaylı bir şekilde incelenmiş ve $B_{z}, J_{x}$ ve $J_{y}$ ifadeleri arasında analitik bir ilişki kurulmuştur. Bu çalışma $B_{z}{ }^{\prime} \operatorname{nin} J_{x}$ ve $J_{y}$ 'deki değişimlere göstediği duyarlılığın anlaşılmasını daha kolay hale getirmiştir. Diğer bir yandan, tek yöndeki manyetik akı yoğunluğunu kullanan yeni bir MRCDI geriçatım algoritması da bu tez kapsamında önerilmiştir. Literatürdeki tekrarlamalı FT-MRCDI algoritması bu çalışmada gerçekleştirilmiştir. Önerilen algoritma ve tekrarlamalı FT-MRCDI algoritması benzetim modelleri ve 
deneysel modeller ile sınanmıştır. Benzetim modellerinde, önerilen algoritma kullanılarak elde edilen akım yoğunluğu görüntülerindeki hata değişik SNR seviyeleri için $0.27 \%$ ve $23.00 \%$ arasında bulunmuştur. Bu hata oranları tekrarlamalı FT-MRCDI algoritması için ise $7.41 \%$ ve $37.45 \%$ arasında değişmektedir. Önerilen algoritma geriçatım süresi bakımından tekrarlamalı FT-MRCDI algoritmasına önemli bir üstünlük kurmuştur. Deneysel modellerde ise, klasik MRCDI algoritması, geriçatılmış akım yoğunlukları görsel olarak karşılaştırıldı̆̆ında en iyi sonucu vermiştir. Geriçatılmış akım yoğunluklarının J-substitution algoritmasında kullanılması sonucu elde edilen iletkenlik dağılımları karşılaştırıldığında ise, önerilen algoritma en iyi sonucu veren algoritma olmuştur. Son olarak elde edilen akım yoğunluğu görüntüleri ıraksama teoremi kullanılarak doğrulanmış ve tekrarlamalı FT-MRCDI algoritmasının üstünlüğü görülmüştür.

Anahtar Kelimeler: elektriksel empedans tomografi, manyetik rezonans görüntüleme, akım yoğunluğu görüntüleme 


\section{ACKNOWLEDGMENTS}

First of all, I would like to thank my family for their love, support and understanding in every stage of my life.

I would like to express my deepest gratitude to my supervisor Prof. Dr. Murat Eyüboğlu for his guidance, advice and encouragement throughout the study.

I would also like to thank TUBITAK-BIDEB for their supports for my M.Sc. study.

I also wish to thank my lab mates and friends: Emre, Evren, Rasim, Gökhan, Tankut, Kerim, Alper, Soner, Ümit, Ceren, Ali Reza, Uğur, Feza, Koray, Balkar, Berna, Reyhan for their valuable advices and chats. A special thanks to Emre and Evren for the endless days and nights in the MRI laboratory.

I also wish to thank my friends: Ersin, Merve, Ozan, Ulaş, Can, Saim, Berk, Emre, Mehmet Emin for their support and friendship. Ankara is a more livable city with them. A special thanks to my home mate Ersin for valuable memories.

Finally, very special thanks to Meriç for her support throughout my M.Sc. study. She always believes me more than I do and her love makes everything easier. 


\section{TABLE OF CONTENTS}

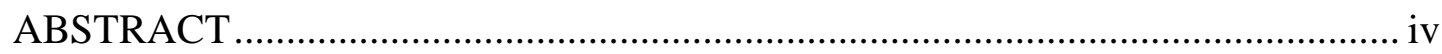

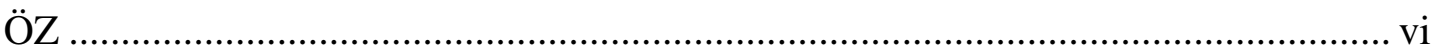

ACKNOWLEDGMENTS _............................................................................ viii

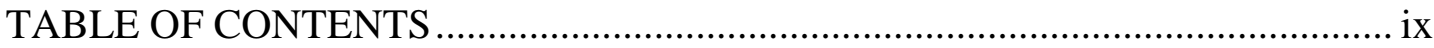

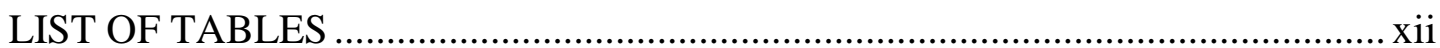

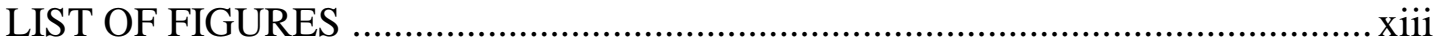

CHAPTER

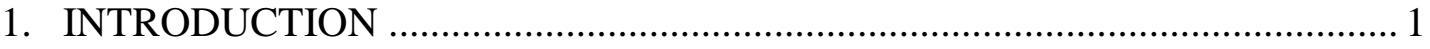

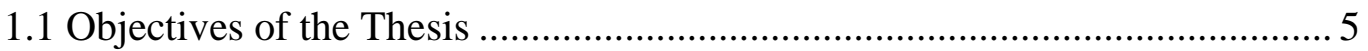

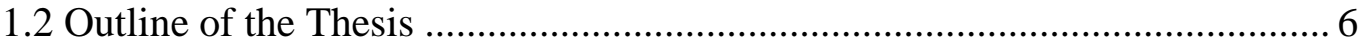

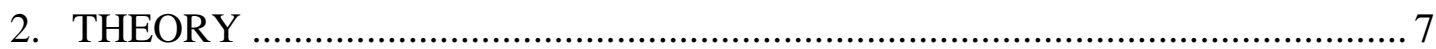

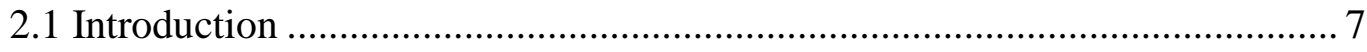

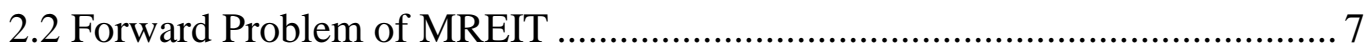

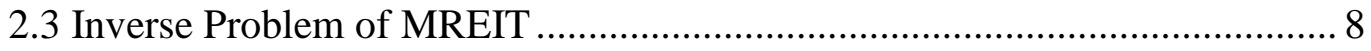

2.4 Extraction of Magnetic Flux Density from MR Images and Classical MRCDI

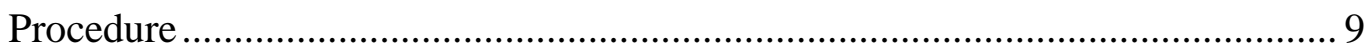

2.5 Experimental Setup in 0.15 T METU-EE MRI System ............................. 13

3. MAGNETIC RESONANCE CURRENT DENSITY IMAGING USING ONE COMPONENT OF MAGNETIC FLUX DENSITY

3.1 Introduction 18

3.2 Analysis of 2D MRCDI Problem 18 
3.2.1 Mathematical Analysis

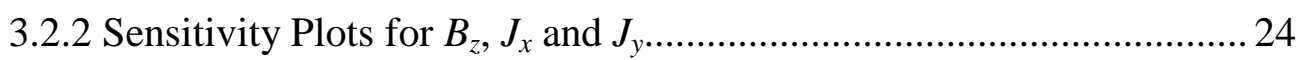

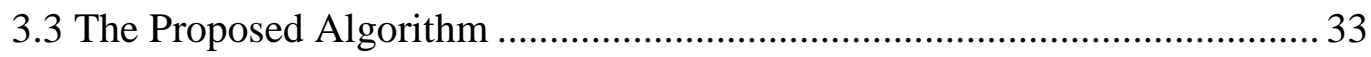

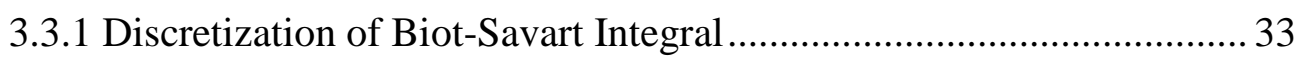

3.3.2 Difference Currents Concept ........................................................... 34

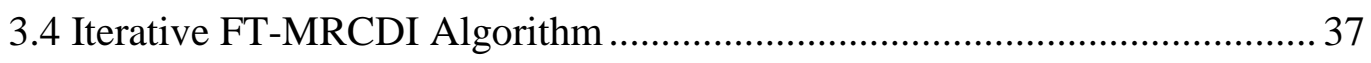

4. SIMULATION AND EXPERIMENTAL MODELS ...................................... 41

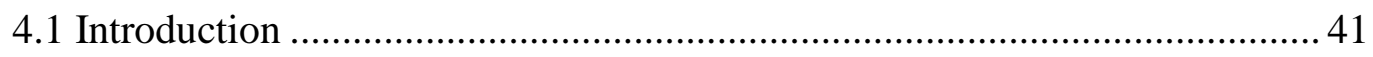

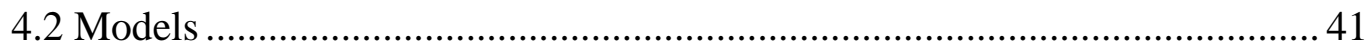

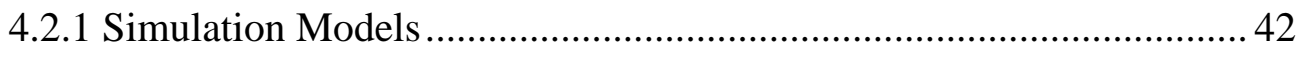

4.2.1.1 Simulation Model 1 …...................................................... 42

4.2.1.2 Simulation Model 2 ….................................................... 43

4.2.1.3 Simulation Model 3 …........................................................ 44

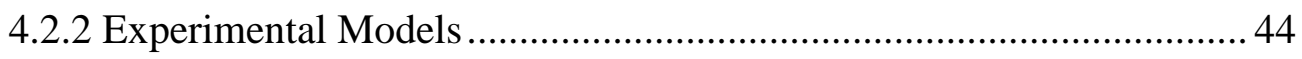

4.2.2.1 Experimental Model 1 ........................................................ 45

4.2.2.2 Experimental Model 2 .................................................. 47

4.2.2.3 Experimental Model 3 ........................................................ 48

4.3 Noise Model for Computer Simulations................................................... 49

4.4 Error Calculation for Simulation Models .................................................... 50

4.5 Error Calculation for Experimental Models .............................................. 50

4.5.1 Verification Using Divergence Theorem............................................. 50

4.5.2 Evaluation in terms of Error in the Reconstructed MREIT Conductivities 51 


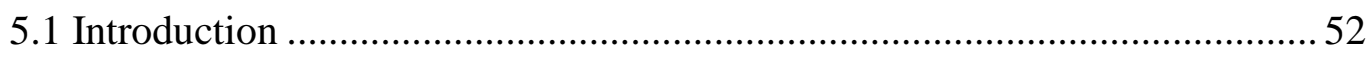

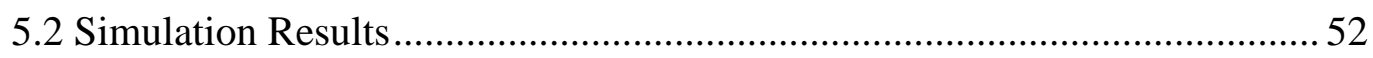

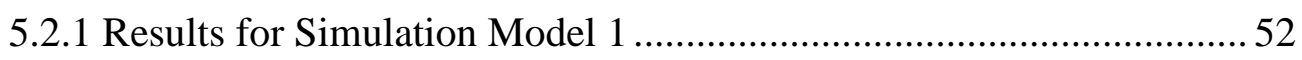

5.2.2 Results for Simulation Model 2 …................................................. 57

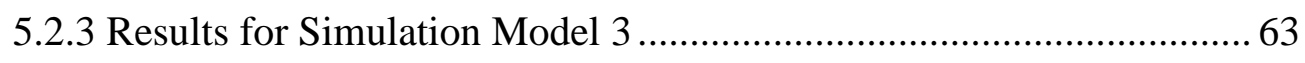

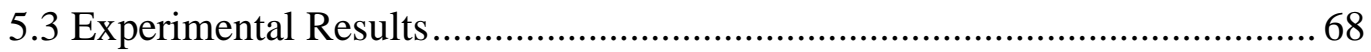

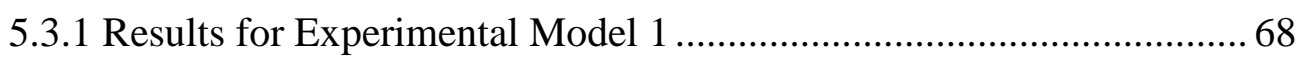

5.3.2 Results for Experimental Model 2 ............................................... 74

5.3.3 Results for Experimental Model 3 .............................................. 77

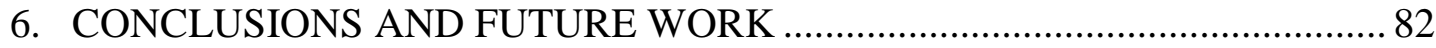

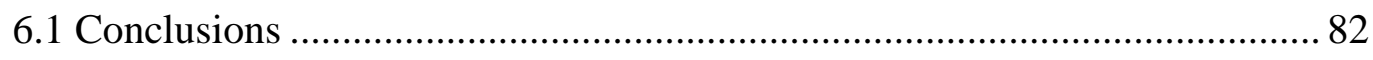

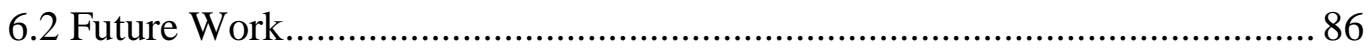

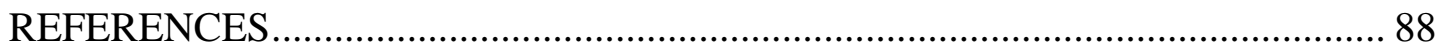

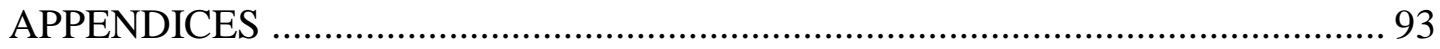

A. COMPUTATION OF CIRCULAR HARMONIC COEFFICIENTS .......93

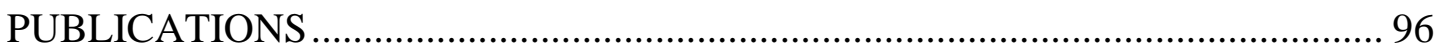




\section{LIST OF TABLES}

\section{TABLES}

Table 4.1 Composition of phantom elements.

Table 5.1 Errors in the reconstructed current density for simulation model 1..........56

Table 5.2 Errors in the reconstructed current density for simulation model 2.......... 62

Table 5.3 Errors in the reconstructed current density for simulation model 3.......... 68

Table 5.4 Calculated standard deviations for experimental model 1...................... 72

Table 5.5 Calculated standard deviations for experimental model 2 ….................... 77

Table 5.6 Calculated standard deviations for experimental model 3...................... 81 


\section{LIST OF FIGURES}

\section{FIGURES}

Figure 2.1 Spin echo pulse sequence.

Figure 2.2 The placement of object inside MRI scanner for measuring all three components of magnetic flux density, a) measuring $B_{z}$, b) measuring $B_{x}$, c) measuring $B_{y}$

Figure 2.3 The experimental setup in METU MRI System.

Figure 2.4 RF coil.

Figure 2.5 Screenshot of MRI software to image $B$

Figure 2.6 Screenshot of MRI software to image $B$

Figure 2.7 Screenshot of MRI software to image $B_{y}$

Figure 3.1 The geometry of the conductor region used for analytical derivations.... 20

Figure 3.2 The geometry of the conductor region prepared in MATLAB 25

Figure 3.3 Analytical solution results of the model for $\sigma_{1}=1 \mathrm{~S} / \mathrm{m}, \sigma_{2}=0.2 \mathrm{~S} / \mathrm{m}, \mathrm{b}=3$ $\mathrm{cm}, \mathrm{a}=9 \mathrm{~cm}$ and $20 \mathrm{~mA}$ current is applied from the electrode on the left and sunk from the electrode on the right, a) The amplitude of the applied current, b) $J_{x}\left(\mathrm{~A} / \mathrm{m}^{2}\right)$ image for vertical current injection, c) $J_{y}\left(\mathrm{~A} / \mathrm{m}^{2}\right)$ image for vertical current injection, d) $B_{z}(\mathrm{~T})$ image for vertical current injection.

Figure $3.4\left\|J_{x}-J_{x}^{\text {uniform }}\right\|$ vs. sigma_ratio plot for various radius_ratio values........ 28

Figure $3.5\left\|J_{x}-J_{x}^{\text {uniform }}\right\|$ vs. radius_ratio plot for various sigma_ratio values........ 28

Figure $3.6\left\|J_{y}-J_{y}^{\text {uniform }}\right\|$ vs. sigma_ratio plot for various radius_ratio values. 29 
Figure $3.7\left\|J_{y}-J_{y}^{u n i f o r m}\right\|$ vs. radius_ratio plot for various sigma_ratio values....

Figure $3.8\left\|B_{z}-B_{z}^{\text {uniform }}\right\|$ vs. sigma_ratio plot for various radius_ratio values. ...... 30

Figure $3.9\left\|B_{z}-B_{z}^{\text {uniform }}\right\|$ vs. radius_ratio plot for various sigma_ratio values........ 30

Figure $3.10 S B_{z} J_{x}$ vs. radius_ratio plot for various sigma_ratio values. 32

Figure $3.11 S B_{z} J_{y}$ vs. radius_ratio plot for various sigma_ratio values 32

Figure 3.12 Flowchart of the proposed algorithm. 37

Figure 3.13 Flowchart of FT-MRCDI algorithm. 40

Figure 4.1 The geometry of Simulation Model 1 42

Figure 4.2 The geometry of Simulation Model 2. 43

Figure 4.3 The geometry of Simulation Model 3. 44

Figure 4.4 Two oblique views of CAD simulations of experimental phantom. 45

Figure 4.5 The experimental phantom. 45

Figure 4.6 a) Top view of the phantom, b) MR magnitude image of the phantom... 47

Figure 4.7 a) Experimental phantom, b) MR magnitude image of the phantom. 48

Figure 4.8 The geometry of Experimental Model 3, a) cross sectional view, b) 3D view. 48

Figure 4.9 a) Top view of the phantom, b) MR magnitude image of the phantom... 49

Figure 5.1 Results for noise-free case (horizontal $20 \mathrm{~mA}$ current injection) a) $B_{z}(\mathrm{~T})$, b) Original $J_{x}\left(\mathrm{~A} / \mathrm{m}^{2}\right)$ distribution, c) Original $J_{y}\left(\mathrm{~A} / \mathrm{m}^{2}\right)$ distribution, d) Reconstructed $J_{x}\left(\mathrm{~A} / \mathrm{m}^{2}\right)$ using the proposed algorithm, e) Reconstructed $J_{y}\left(\mathrm{~A} / \mathrm{m}^{2}\right)$ using the proposed algorithm, f) Reconstructed $J_{x}\left(\mathrm{~A} / \mathrm{m}^{2}\right)$ using Iterative FT-MRCDI algorithm, g) Reconstructed $J_{y}\left(\mathrm{~A} / \mathrm{m}^{2}\right)$ using Iterative FT-MRCDI algorithm..........53

Figure 5.2 Results for SNR 30 case (horizontal 20mA current injection) 
a) $B_{z}(\mathrm{~T})$, b) Original $J_{x}\left(\mathrm{~A} / \mathrm{m}^{2}\right)$ distribution, c) Original $J_{y}\left(\mathrm{~A} / \mathrm{m}^{2}\right)$ distribution,d $)$ Reconstructed $J_{x}\left(\mathrm{~A} / \mathrm{m}^{2}\right)$ using the proposed algorithm, e) Reconstructed $J_{y}\left(\mathrm{~A} / \mathrm{m}^{2}\right)$ using the proposed algorithm, f) Reconstructed $J_{x}\left(\mathrm{~A} / \mathrm{m}^{2}\right)$ using Iterative FT-MRCDI algorithm, g) Reconstructed $J_{y}\left(\mathrm{~A} / \mathrm{m}^{2}\right)$ using Iterative FT-MRCDI algorithm.

Figure 5.3 Results for SNR 13 case (horizontal $20 \mathrm{~mA}$ current injection) a) $B_{z}(\mathrm{~T})$, b) Original $J_{x}\left(\mathrm{~A} / \mathrm{m}^{2}\right)$ distribution, c) Original $J_{y}\left(\mathrm{~A} / \mathrm{m}^{2}\right)$ distribution, d) Reconstructed $J_{x}\left(\mathrm{~A} / \mathrm{m}^{2}\right)$ using the proposed algorithm, e) Reconstructed $J_{y}\left(\mathrm{~A} / \mathrm{m}^{2}\right)$ using the proposed algorithm, f) Reconstructed $J_{x}\left(\mathrm{~A} / \mathrm{m}^{2}\right)$ using Iterative FT-MRCDI algorithm, g) Reconstructed $J_{y}\left(\mathrm{~A} / \mathrm{m}^{2}\right)$ using Iterative FT-MRCDI algorithm.

Figure 5.4 z-component of magnetic flux density, $B_{z}(\mathrm{~T})$ a) For horizontal current injection pattern, b) For vertical current injection pattern.

Figure 5.5 Reconstructed $J_{x}$ for different noise levels (horizontal $20 \mathrm{~mA}$ current injection) a) Original $J_{x}\left(\mathrm{~A} / \mathrm{m}^{2}\right)$ distribution, b) Reconstructed $J_{x}\left(\mathrm{~A} / \mathrm{m}^{2}\right)$ using the proposed algorithm for noise-free case, c) Reconstructed $J_{x}\left(\mathrm{~A} / \mathrm{m}^{2}\right)$ using Iterative FT-MRCDI algorithm for noise-free case, d) Reconstructed $J_{x}\left(\mathrm{~A} / \mathrm{m}^{2}\right)$ using the proposed algorithm for SNR30 case, e) Reconstructed $J_{x}\left(\mathrm{~A} / \mathrm{m}^{2}\right)$ using Iterative FTMRCDI algorithm for SNR 30 case, f) Reconstructed $J_{x}\left(\mathrm{~A} / \mathrm{m}^{2}\right)$ using the proposed algorithm for SNR13 case, g) Reconstructed $J_{x}\left(\mathrm{~A} / \mathrm{m}^{2}\right)$ using Iterative FT-MRCDI algorithm for SNR 13 case.

Figure 5.6 Reconstructed $J_{y}$ for different noise levels (horizontal $20 \mathrm{~mA}$ current injection) a) Original $J_{y}\left(\mathrm{~A} / \mathrm{m}^{2}\right)$ distribution, b) Reconstructed $J_{y}\left(\mathrm{~A} / \mathrm{m}^{2}\right)$ using the proposed algorithm for noise-free case, c) Reconstructed $J_{y}\left(\mathrm{~A} / \mathrm{m}^{2}\right)$ using Iterative FT-MRCDI algorithm for noise-free case, d) Reconstructed $J_{y}\left(\mathrm{~A} / \mathrm{m}^{2}\right)$ using the proposed algorithm for SNR30 case, e) Reconstructed $J_{y}\left(\mathrm{~A} / \mathrm{m}^{2}\right)$ using Iterative FTMRCDI algorithm for SNR 30 case, f) Reconstructed $J_{y}\left(\mathrm{~A} / \mathrm{m}^{2}\right)$ using the proposed algorithm for SNR13 case, g) Reconstructed $J_{y}\left(\mathrm{~A} / \mathrm{m}^{2}\right)$ using Iterative FT-MRCDI algorithm for SNR 13 case.

Figure 5.7 Reconstructed $J_{x}$ for different noise levels (vertical $20 \mathrm{~mA}$ current injection) a) Original $J_{x}\left(\mathrm{~A} / \mathrm{m}^{2}\right)$ distribution, b) Reconstructed $J_{x}\left(\mathrm{~A} / \mathrm{m}^{2}\right)$ using the proposed algorithm for noise-free case, c) Reconstructed $J_{x}\left(\mathrm{~A} / \mathrm{m}^{2}\right)$ using Iterative FT-MRCDI algorithm for noise-free case, d) Reconstructed $J_{x}\left(\mathrm{~A} / \mathrm{m}^{2}\right)$ using the proposed algorithm for SNR30 case, e) Reconstructed $J_{x}\left(\mathrm{~A} / \mathrm{m}^{2}\right)$ using Iterative FTMRCDI algorithm for SNR 30 case, f) Reconstructed $J_{x}\left(\mathrm{~A} / \mathrm{m}^{2}\right)$ using the proposed algorithm for SNR13 case, g) Reconstructed $J_{x}\left(\mathrm{~A} / \mathrm{m}^{2}\right)$ using Iterative FT-MRCDI algorithm for SNR 13 case. 60

Figure 5.8 Reconstructed $J_{y}$ for different noise levels $(20 \mathrm{~mA}$ current is injected vertically and note that direction of positive y-axis is taken as negative) a) Original $J_{y}$ $\left(\mathrm{A} / \mathrm{m}^{2}\right)$ distribution, b) Reconstructed $J_{y}\left(\mathrm{~A} / \mathrm{m}^{2}\right)$ using the proposed algorithm for noise-free case, c) Reconstructed $J_{y}\left(\mathrm{~A} / \mathrm{m}^{2}\right)$ using Iterative FT-MRCDI algorithm for noise-free case, d) Reconstructed $J_{y}\left(\mathrm{~A} / \mathrm{m}^{2}\right)$ using the proposed algorithm for SNR30 case, e) Reconstructed $J_{y}\left(\mathrm{~A} / \mathrm{m}^{2}\right)$ using Iterative FT-MRCDI algorithm for SNR 30 
case, f) Reconstructed $J_{y}\left(\mathrm{~A} / \mathrm{m}^{2}\right)$ using the proposed algorithm for SNR13 case, g) Reconstructed $J_{y}\left(\mathrm{~A} / \mathrm{m}^{2}\right)$ using Iterative FT-MRCDI algorithm for SNR 13 case..... 61

Figure 5.9 z-component of magnetic flux density, $B_{z}(\mathrm{~T})$ a) For horizontal current injection pattern, b) For vertical current injection pattern. 63

Figure 5.10 Reconstructed $J_{x}$ for different noise levels (horizontal $20 \mathrm{~mA}$ current injection) a) Original $J_{x}\left(\mathrm{~A} / \mathrm{m}^{2}\right)$ distribution, b) Reconstructed $J_{x}\left(\mathrm{~A} / \mathrm{m}^{2}\right)$ using the proposed algorithm for noise-free case, c) Reconstructed $J_{x}\left(\mathrm{~A} / \mathrm{m}^{2}\right)$ using Iterative FT-MRCDI algorithm for noise-free case, d) Reconstructed $J_{x}\left(\mathrm{~A} / \mathrm{m}^{2}\right)$ using the proposed algorithm for SNR30 case, e) Reconstructed $J_{x}\left(\mathrm{~A} / \mathrm{m}^{2}\right)$ using Iterative FTMRCDI algorithm for SNR 30 case, f) Reconstructed $J_{x}\left(\mathrm{~A} / \mathrm{m}^{2}\right)$ using the proposed algorithm for SNR13 case, g) Reconstructed $J_{x}\left(\mathrm{~A} / \mathrm{m}^{2}\right)$ using Iterative FT-MRCDI algorithm for SNR 13 case. .64

Figure 5.11 Reconstructed $J_{y}$ for different noise levels (horizontal $20 \mathrm{~mA}$ current injection) a) Original $J_{y}\left(\mathrm{~A} / \mathrm{m}^{2}\right)$ distribution, b) Reconstructed $J_{y}\left(\mathrm{~A} / \mathrm{m}^{2}\right)$ using the proposed algorithm for noise-free case, c) Reconstructed $J_{y}\left(\mathrm{~A} / \mathrm{m}^{2}\right)$ using Iterative FT-MRCDI algorithm for noise-free case, d) Reconstructed $J_{y}\left(\mathrm{~A} / \mathrm{m}^{2}\right)$ using the proposed algorithm for SNR30 case, e) Reconstructed $J_{y}\left(\mathrm{~A} / \mathrm{m}^{2}\right)$ using Iterative FTMRCDI algorithm for SNR 30 case, f) Reconstructed $J_{y}\left(\mathrm{~A} / \mathrm{m}^{2}\right)$ using the proposed algorithm for SNR13 case, g) Reconstructed $J_{y}\left(\mathrm{~A} / \mathrm{m}^{2}\right)$ using Iterative FT-MRCDI algorithm for SNR 13 case. 65

Figure 5.12 Reconstructed $J_{x}$ for different noise levels (vertical $20 \mathrm{~mA}$ current injection) a) Original $J_{x}\left(\mathrm{~A} / \mathrm{m}^{2}\right)$ distribution, b) Reconstructed $J_{x}\left(\mathrm{~A} / \mathrm{m}^{2}\right)$ using the proposed algorithm for noise-free case, c) Reconstructed $J_{x}\left(\mathrm{~A} / \mathrm{m}^{2}\right)$ using Iterative FT-MRCDI algorithm for noise-free case, d) Reconstructed $J_{x}\left(\mathrm{~A} / \mathrm{m}^{2}\right)$ using the proposed algorithm for SNR30 case, e) Reconstructed $J_{x}\left(\mathrm{~A} / \mathrm{m}^{2}\right)$ using Iterative FTMRCDI algorithm for SNR 30 case, f) Reconstructed $J_{x}\left(\mathrm{~A} / \mathrm{m}^{2}\right)$ using the proposed algorithm for SNR13 case, g) Reconstructed $J_{x}\left(\mathrm{~A} / \mathrm{m}^{2}\right)$ using Iterative FT-MRCDI algorithm for SNR 13 case. 66

Figure 5.13 Reconstructed $J_{y}$ for different noise levels (20 mA current is injected vertically and note that direction of positive y-axis is taken as negative) a) Original $J_{y}$ $\left(\mathrm{A} / \mathrm{m}^{2}\right)$ distribution, b) Reconstructed $J_{y}\left(\mathrm{~A} / \mathrm{m}^{2}\right)$ using the proposed algorithm for noise-free case, c) Reconstructed $J_{y}\left(\mathrm{~A} / \mathrm{m}^{2}\right)$ using Iterative FT-MRCDI algorithm for noise-free case, d) Reconstructed $J_{y}\left(\mathrm{~A} / \mathrm{m}^{2}\right)$ using the proposed algorithm for SNR30 case, e) Reconstructed $J_{y}\left(\mathrm{~A} / \mathrm{m}^{2}\right)$ using Iterative FT-MRCDI algorithm for SNR 30 case, f) Reconstructed $J_{y}\left(\mathrm{~A} / \mathrm{m}^{2}\right)$ using the proposed algorithm for SNR13 case, $\mathrm{g}$ ) Reconstructed $J_{y}\left(\mathrm{~A} / \mathrm{m}^{2}\right)$ using Iterative FT-MRCDI algorithm for SNR 13 case..... 67

Figure 5.14 Magnetic flux density in z direction, $B_{z}(\mathrm{~T})$, a) For horizontal current injection pattern, b) For vertical current injection pattern. 69

Figure 5.15 Results for horizontal current injection pattern, a) Reconstructed $J_{x}$ 
$\left(\mathrm{A} / \mathrm{m}^{2}\right)$ using three components of $\vec{B}$, b) Reconstructed $J_{y}\left(\mathrm{~A} / \mathrm{m}^{2}\right)$ using three components of $\vec{B}$, c) Reconstructed $J_{x}\left(\mathrm{~A} / \mathrm{m}^{2}\right)$ using the proposed algorithm, d) Reconstructed $J_{y}\left(\mathrm{~A} / \mathrm{m}^{2}\right)$ using the proposed algorithm, e) Reconstructed $J_{x}\left(\mathrm{~A} / \mathrm{m}^{2}\right)$ using Iterative FT-MRCDI algorithm, f) Reconstructed $J_{y}\left(\mathrm{~A} / \mathrm{m}^{2}\right)$ using Iterative FTMRCDI algorithm.

Figure 5.16 Results for vertical current injection pattern, (note that direction of positive $y$-axis is taken as negative) a) Reconstructed $J_{x}(\mathrm{~A} / \mathrm{m} 2)$ using three components of $\vec{B}$, b) Reconstructed $J_{y}(\mathrm{~A} / \mathrm{m} 2)$ using three components of $\vec{B}$, c) Reconstructed $J_{x}(\mathrm{~A} / \mathrm{m} 2)$ using the proposed algorithm, d) Reconstructed $J_{y}(\mathrm{~A} / \mathrm{m} 2)$ using the proposed algorithm, e) Reconstructed $J_{x}(\mathrm{~A} / \mathrm{m} 2)$ using Iterative FT-MRCDI algorithm, f) Reconstructed $J_{y}(\mathrm{~A} / \mathrm{m} 2)$ using Iterative FT-MRCDI algorithm. 71

Figure 5.17 Reconstructed conductivity images with J-substitution algorithm after 5 iterations, a) Original distribution, b) Reconstructed conductivity image using $\vec{J}$ obtained from the classical MRCDI algorithm, c) Reconstructed conductivity image using $\vec{J}$ obtained from the proposed algorithm, d) Reconstructed conductivity image using $\vec{J}$ obtained from the iterative FT-MRCDI algorithm. .73

Figure 5.18 Magnetic flux density in $\mathrm{z}$ direction, $B_{z}(\mathrm{~T})$, a) For horizontal current injection pattern, b) For vertical current injection pattern.

Figure 5.19 Results for horizontal current injection pattern, a) Reconstructed $J_{x}$ $\left(\mathrm{A} / \mathrm{m}^{2}\right)$ using three components of $\vec{B}$, b) Reconstructed $J_{y}\left(\mathrm{~A} / \mathrm{m}^{2}\right)$ using three components of $\vec{B}$, c) Reconstructed $J_{x}\left(\mathrm{~A} / \mathrm{m}^{2}\right)$ using the proposed algorithm, d) Reconstructed $J_{y}\left(\mathrm{~A} / \mathrm{m}^{2}\right)$ using the proposed algorithm, e) Reconstructed $J_{x}\left(\mathrm{~A} / \mathrm{m}^{2}\right)$ using Iterative FT-MRCDI algorithm, f) Reconstructed $J_{y}\left(\mathrm{~A} / \mathrm{m}^{2}\right)$ using Iterative FTMRCDI algorithm. .75

Figure 5.20 Results for vertical current injection pattern, (note that direction of positive $\mathrm{y}$-axis is taken as negative) a) Reconstructed $J_{x}\left(\mathrm{~A} / \mathrm{m}^{2}\right)$ using three components of $\vec{B}$, b) Reconstructed $J_{y}\left(\mathrm{~A} / \mathrm{m}^{2}\right)$ using three components of $\vec{B}$, c) Reconstructed $J_{x}\left(\mathrm{~A} / \mathrm{m}^{2}\right)$ using the proposed algorithm, d) Reconstructed $J_{y}\left(\mathrm{~A} / \mathrm{m}^{2}\right)$ using the proposed algorithm, e) Reconstructed $J_{x}\left(\mathrm{~A} / \mathrm{m}^{2}\right)$ using Iterative FT-MRCDI algorithm, f) Reconstructed $J_{y}\left(\mathrm{~A} / \mathrm{m}^{2}\right)$ using Iterative FT-MRCDI algorithm. 76

Figure 5.21 Magnetic flux density in $\mathrm{z}$ direction, $B_{z}(\mathrm{~T})$, a) For horizontal current injection pattern, b) For vertical current injection pattern.

Figure 5.22 Results for horizontal current injection pattern, a) Reconstructed $J_{x}$ $\left(\mathrm{A} / \mathrm{m}^{2}\right)$ using three components of $\vec{B}$, b) Reconstructed $J_{y}\left(\mathrm{~A} / \mathrm{m}^{2}\right)$ using three components of $\vec{B}$, c) Reconstructed $J_{x}\left(\mathrm{~A} / \mathrm{m}^{2}\right)$ using the proposed algorithm, d) Reconstructed $J_{y}\left(\mathrm{~A} / \mathrm{m}^{2}\right)$ using the proposed algorithm, e) Reconstructed $J_{x}\left(\mathrm{~A} / \mathrm{m}^{2}\right)$ using Iterative FT-MRCDI algorithm, f) Reconstructed $J_{y}\left(\mathrm{~A} / \mathrm{m}^{2}\right)$ using Iterative FT- 
Figure 5.23 Results for vertical current injection pattern, (note that direction of positive $\mathrm{y}$-axis is taken as negative) a) Reconstructed $J_{x}\left(\mathrm{~A} / \mathrm{m}^{2}\right)$ using three components of $\vec{B}$, b) Reconstructed $J_{y}\left(\mathrm{~A} / \mathrm{m}^{2}\right)$ using three components of $\vec{B}$, c) Reconstructed $J_{x}\left(\mathrm{~A} / \mathrm{m}^{2}\right)$ using the proposed algorithm, d) Reconstructed $J_{y}\left(\mathrm{~A} / \mathrm{m}^{2}\right)$ using the proposed algorithm, e) Reconstructed $J_{x}\left(\mathrm{~A} / \mathrm{m}^{2}\right)$ using Iterative FT-MRCDI algorithm, f) Reconstructed $J_{y}\left(\mathrm{~A} / \mathrm{m}^{2}\right)$ using Iterative FT-MRCDI algorithm. 80 


\section{CHAPTER 1}

\section{INTRODUCTION}

The properties of biological tissues differ among tissues, which make the imaging of human body possible. One of these distinctive properties is the electrical conductivity of the tissues. Electrical conductivity of the tissues varies among tissues and it also changes with physiological and pathological state of a tissue [1], [2]. The electrical impedance of a tissue is modeled by Philippson [3] in terms of a resistorcapacitor pair. Electrical impedance tomography (EIT) is a non-invasive imaging technique which is proposed to image electrical conductivity distribution inside a volume conductor.

The idea of generating electrical conductivity distribution images of the body was first proposed by Henderson and Webster [4] in 1978. They presented the first electrical impedance images. However, the first published tomographic images were obtained by Brown and Barber [5]. Interested reader in the history of EIT is encouraged to read the review article by Brown [6].

Various current injection and voltage measurement schemes for EIT are proposed in literature. The most widely used scheme is Sheffield's scheme [7] where 16 electrodes are used and 104 independent transfer impedance measurements are made. In order to generate different current density distribution inside the volume conductor, induced current EIT method is proposed [8]. In this method, the current is induced via a coil placed around a body. Gençer et al presented the mathematical principles of induced current EIT and extend the studies by introducing contactless measurement system [9], [10].

Brown mentioned the possible applications of EIT in healthcare as gastric 
measurements (gastric secretion and emptying), pulmonary measurements, head imaging, breast imaging [6].

Although EIT has several advantages over other methods as it is a relatively cheap technique, it has fast data collection procedure and it has no hazards to human body [6], EIT has also many drawbacks that limits its use for clinical purposes. The main drawback of EIT is low spatial resolution and space-dependent quantitative accuracy of reconstructed images [11]. The low spatial resolution is because of the fact that the sensitivity of peripheral voltage measurements to conductivity changes in different regions is not the same. Therefore, extra information obtained directly from the measurements inside the volume conductor is needed to overcome low spatial resolution problem of EIT.

In 1988, Joy et al developed a novel imaging technique to reconstruct current density distribution inside a volume conductor containing nuclear magnetic resonance (NMR) active nuclei by using Magnetic Resonance Imaging (MRI) system [12]. This technique was named as Magnetic Resonance Current Density Imaging (MRCDI). This technique enabled the monitoring of current density distribution with high resolution. In MRCDI, current is applied to the phantom by the electrodes placed on the surface of the phantom and generated magnetic flux density due to externally applied currents is measured by MRI scanner. Then, the current density distribution is calculated by using the Maxwell equation for the static electric field:

$$
\nabla \times \vec{B}=\mu_{0} \vec{J}
$$

As it can be seen in Equation (1.1), all three components of magnetic flux density should be measured to calculate current density distribution. Since MRI scanner can only measure the component of magnetic flux density parallel with the main magnetic field, the phantom should be rotated inside MRI scanner to measure all three components.

The main advantage of MRCDI technique is that it allows to measure magnetic flux density at the points where the currents flow. Therefore, the magnetic flux density created by small currents can also be measured. As a result, high resolution current density images are obtained. The development of MRCDI technique provides 
valuable information in many biomedical engineering applications as determination of hazardous electric currents, electrode design for various applications, calculation of lead sensitivity maps of biopotential recording electrodes and improvement of spatial resolution of conductivity images in EIT [13].

In 1992, Zhang proposed a new algorithm that uses current density distribution obtained from MRCDI with conventional EIT voltage measurements to reconstruct electrical conductivity distribution inside a volume conductor [14]. In his algorithm, Zhang used the fact that potentials on the surface of volume conductor are known from conventional EIT measurements. Hence, any potential difference measured on the surface can be represented as

$$
\Delta \Phi=\int_{C} \rho \vec{J} \cdot d \vec{l}
$$

where $\vec{J}$ is the current density, $\rho$ is the resistivity and $\Delta \Phi$ is the voltage difference between two points on surface. Since voltage difference and current density are known, solving the linear inverse problem gives the conductivity distribution. This novel algorithm is named as Magnetic Resonance Electrical Impedance Tomography (MREIT).

After Zhang proposed the first MREIT algorithm, MREIT has been studied by many research groups. In the literature, MREIT reconstruction algorithms can be classified in two groups: algorithms that use magnetic flux density, $\vec{B}$, directly and algorithms that use current density distribution, $\vec{J}$, obtained from measured $\vec{B}$.

Some of the $J$-based MREIT reconstruction algorithms proposed in the literature are Integration along Equipotential Lines, Integration along Cartesian Grid Lines, Solution of a Linear Equation System using Finite Differences [15], EquipotentialProjection algorithm [16], J-substitution algorithm [17] and J-substitution and Filtered Equipotential-Projection Based Hybrid Reconstruction Algorithm [18]. In 2009, all these $J$-based MREIT reconstruction algorithms were implemented and their performances were evaluated by Boyacıoğlu and Eyüboğlu [18].

Some of the $B$-based MREIT reconstruction algorithms proposed in the literature are Harmonic $B_{z}$ algorithm [19], Variational Gradient $B_{z}$ algorithm [20], Sensitivity 
matrix algorithm [21], Algebraic Reconstruction algorithm [15]. In 2009, all these $B$ based MREIT reconstruction algorithms were implemented and their performances were evaluated by Eker and Eyüboğlu [22].

As it was mentioned before, the phantom should be rotated inside MRI scanner to measure all three components of $\vec{B}$ in MRCDI technique since MRI scanner can only measure the component of $\vec{B}$ parallel with the main magnetic field. However, rotating the phantom inside the magnet is not trivial even for small size phantoms. This problem is one of the drawbacks of MRCDI that limits its use in clinical applications. In 1989, before Joy et al conducted the first MRCDI experiment [12], Roth et al showed that the current density distributions restricted to a two dimensional plane can be reconstructed by using only the component of $\vec{B}$ perpendicular to the imaging plane, $B_{z}[23]$. They assumed that $B_{z}$ was measured by a superconducting quantum interference device (SQUID). First, they obtained the Fourier Transform of $B_{z}$. Then, by using Biot-Savart law and the fact that the divergence of current density vanishes for quasistatic currents, they solved the inverse problem which gives $J_{x}$ and $J_{y}$. In 1990, Pesikan et al used the same reconstruction technique as Roth et al used to reconstruct 2D current density distribution. However, they measured $B_{z}$ by MRI scanner [24]. In 2003, Seo et al proposed a new current density reconstruction algorithm known as Harmonic $B_{Z}$ algorithm which also uses only one component of $\vec{B}$ [19]. In this algorithm, the iterations are started with assuming an initial conductivity distribution. The conductivity distribution is updated at the successive iterations by an update equation until the error reduces below a predetermined tolerance value. Finally, the current density distribution is calculated by solving the boundary value problem. In 2006, İder proposed a novel reconstruction algorithm to reconstruct 2D current density distribution [25]. He used the Fourier Transform and spatial filtering techniques as Roth et al did. Furthermore, he introduced difference current concept to apply the technique on the phantom where current is applied by the electrodes placed on its boundaries. Difference currents are obtained by subtracting $\vec{J}$ calculated for a uniform conductivity by finite element method from actual $\vec{J}$. Divergence of the difference current vanishes. In this algorithm, first difference magnetic flux density is 
calculated by subtracting the magnetic flux density for uniform currents from the measured magnetic flux density. Then, difference currents are calculated by solving inverse problem. Finally, $\vec{J}$ is obtained by adding difference currents to the uniform currents.

\subsection{Objectives of the Thesis}

MREIT has been studied by many research groups since Zhang proposed the first MREIT algorithm in 1992 [14]. As it was mentioned before, $\vec{J}$ must be calculated from measured $\vec{B}$ for $J$-based MREIT reconstruction algorithms. In classical MRCDI technique, all three components of $\vec{B}$ are used to reconstruct $\vec{J}$. Hence, object rotation is necessary to measure all three components of $\vec{B}$. The algorithms that eliminate the need for object rotation were given in previous section. In Harmonic $B_{z}$ algorithm, noise performance of the algorithm is poor since the algorithm calculates Laplacian of measured $B_{z}$. Therefore, performance of the algorithm on experimental phantoms is poor for low SNR MRI Systems. The MRCDI algorithms, which use Fourier Transform, require the measurement of $\vec{B}$ outside of the phantom to obtain a well-defined Fourier Transform. However, measuring magnetic flux density outside the phantom is not possible in an MRI scanner. Hence, post-processing of measured $B_{z}$ data is required. As a result, there is a need for novel MRCDI reconstruction algorithms that use only one component of measured $\vec{B}$ directly. Also, performance of these algorithms on experimental data is essential for the improvement of MRCDI technique. Hence, objectives of this thesis are:

- To analyze the relation between one component of magnetic flux density and current density distribution orthogonal to that component of magnetic flux density,

- To develop novel reconstruction algorithm using one component of magnetic flux density for MRCDI,

- To evaluate the performance of the developed algorithms on both simulated and experimental data, 
- To compare the performance of the developed algorithm with other algorithms proposed in literature,

In order to compare the performance of the proposed algorithm, the iterative FTMRCDI algorithm is also implemented in this thesis. This algorithm is chosen since the experiments are conducted in a low SNR MRI System and the iterative FTMRCDI has a better noise performance compared to other reconstruction algorithms.

\subsection{Outline of the Thesis}

In Chapter 2, the forward and inverse problems of MREIT are explained. Moreover, the extraction of magnetic flux density from MR images and the classical MRCDI procedure to obtain current density distribution by using three components of magnetic flux density are given in detail in this chapter. Then, the experimental setup in 0.15T METU-EE MRI System is explained. In Chapter 3, the inverse problem of Magnetic Resonance Current Density Imaging (MRCDI), which relates magnetic flux density to current density, will be investigated in detail. The reconstruction algorithms are also explained with their formulations in this chapter. In Chapter 4, simulation and experimental models used to evaluate reconstruction performance of the MRCDI algorithms are given. The error calculation method for both models is also formulated. In Chapter 5, simulation and experimental results for reconstruction algorithms are given. Furthermore, the comparison of performance of the reconstruction algorithms is carried out in this chapter. Finally, Chapter 6 includes the conclusion and future works. 


\section{CHAPTER 2}

\section{THEORY}

\subsection{Introduction}

In this chapter, the forward problem of MREIT, which includes the definition and governing equations, is explained in detail. Inverse problem of MREIT is explained afterwards. The extraction of magnetic flux density from MR images and the classical MRCDI procedure to obtain current density distribution by using three components of magnetic flux density are also given in detail in this chapter. Finally, experimental setup in 0.15 T METU-EE MRI System is explained.

\subsection{Forward Problem of MREIT}

The forward problem of MREIT consists of the calculation of peripheral surface voltages and magnetic flux density distribution for a subject to be imaged. The relation between the conductivity, $\sigma$, and the potential field, $\Phi$, is defined by Poisson's equation as:

$$
\nabla \cdot(\sigma \nabla \Phi)=0
$$

The electric current on the boundary of the imaging region is represented with the Neumann boundary conditions:

$$
\sigma \frac{\partial \Phi}{\partial n}=\left\{\begin{array}{l}
J \quad \text { on positive current electrode } \\
-J \text { on negative current electrode } \\
0 \quad \text { elsewhere }
\end{array}\right.
$$

where $\mathrm{n}$ is the outward normal at the outer boundary. The electrical field distribution 
can be calculated from potential distribution as

$$
\vec{E}=-\nabla \Phi
$$

and the corresponding current density distribution is obtained as

$$
\vec{J}=\sigma \vec{E}
$$

The relation between $\vec{J}$ and magnetic flux density, $\vec{B}$, is given by Biot-Savart equation as

$$
\vec{B}=\frac{\mu_{0}}{4 \pi} \int \frac{(\vec{J} d V) \times \vec{r}}{r^{3}}
$$

where $\mu_{0}$ is the permeability of free space, $r$ is the distance between field and source points, $\vec{r}$ is the unit vector pointing from source to field point. The Biot-Savart law enables the calculation of magnetic flux density generated due to current density distribution on any slice. Equations (2.1)-(2.5) describe the relation between the conductivity distribution and magnetic flux density.

Analytical solution of the forward problem is restricted to problems with special geometries. Hence, finite element or boundary element methods are used generally for the solution of the forward problem.

\subsection{Inverse Problem of MREIT}

The inverse problem of MREIT includes reconstruction of the conductivity distribution inside a volume conductor by using measured magnetic flux density and peripheral surface measurements. Solution of the MREIT inverse problem was first proposed by Zhang [14]. In his algorithm, Zhang used the fact that potentials on the surface of a volume conductor are known from conventional EIT measurements. Hence, any potential difference measured on the surface can be represented as

$$
\Delta \Phi=\int_{C} \rho \vec{J} \cdot d \vec{l}
$$

where $\vec{J}$ is the current density, $\rho$ is the resistivity and $\Delta \Phi$ is the potential difference 
between two points on surface. Since potential difference and current density distribution are known, the conductivity distribution is obtained by solving the linear inverse problem.

MREIT reconstruction algorithms can be grouped in two categories:

1. $\vec{B}$-based algorithms (Algorithms that use $\vec{B}$ directly),

2. $\vec{J}$-based algorithms (Algorithms that use $\vec{J}$ obtained from $\vec{B}$ )

The $\vec{J}$-based MREIT reconstruction algorithms proposed in the literature were implemented and their performances were evaluated by Boyacıoğlu and Eyüboğlu [18]. The $\vec{B}$-based MREIT reconstruction algorithms proposed in the literature were implemented and their performances were evaluated by Eker and Eyüboğlu [22].

\subsection{Extraction of Magnetic Flux Density from MR Images and Classical MRCDI Procedure}

It is known that the currents flowing in a volume conductor generates a magnetic flux density. The generated magnetic flux density can be measured by using magnetic resonance imaging techniques if the conductor region contains NMR active nuclei. The magnetic flux density is extracted from the MR images that are obtained by using appropriate pulse sequences. However, the component of magnetic flux density parallel with the main magnetic field of MRI system can be measured only. Because, only the component of magnetic flux density, which is in the same direction with the main magnetic field, accumulates a phase in the acquired MRI signal [26].

If a noise free MRI data without spin relaxation is assumed, the acquired MRI signal in a spin echo imaging experiment with pulse sequence shown in Figure 2.1 can be expressed as

$$
S\left(k_{x}, k_{y}, t\right)=\iint_{x} M(x, y) \exp \left\{j\left[\gamma B t+k_{x} x+k_{y} y+\theta_{c}\right]\right\} d x d y
$$

where $M(x, y)$ is the continuous real transverse magnetization, $B$ is the inhomogeneity component of the main magnetic field and $\theta_{c}$ is a constant phase due to 
instrumentation and receiver circuits, $k_{x}=\gamma G_{x} t$ and $k_{y}=\gamma G_{y} t_{y}$, where $G_{x}$ and $G_{y}$ are the frequency and phase encoding gradient strengths, respectively. $t$ is the data acquisition time and $t_{y}$ is the duration of $G_{y}$ gradient pulse and $\gamma$ is the gyromagnetic ratio.

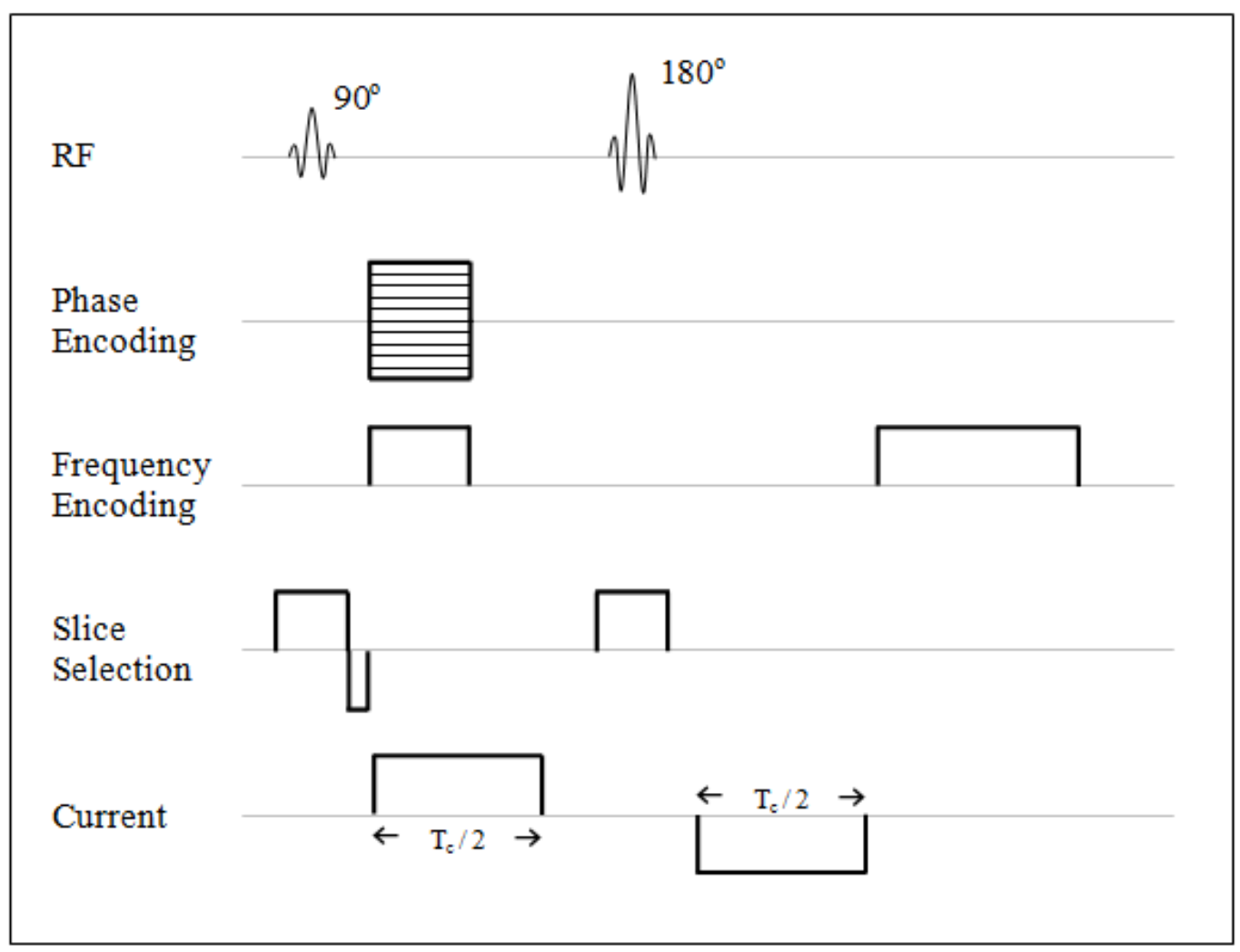

Figure 2.1 Spin echo pulse sequence.

When current synchronized with spin echo pulse sequence is applied to the conductor region, the component of magnetic flux density parallel with the main magnetic field of MRI system accumulates a phase in the acquired signal as mentioned before. The acquired signal can be expressed as

$$
S\left(k_{x}, k_{y}, t\right)=\int_{x} \int_{y} M(x, y) \exp \left\{j\left[\gamma B t+k_{x} x+k_{y} y+\theta_{c}+\gamma B_{j}(x, y) T_{c}\right]\right\} d x d y
$$

Here, $B_{j}(x, y)$ is the component of magnetic flux density parallel with the main 
magnetic field and $T_{c}$ is the duration of the applied current. Complex MR images for current applied case and no-current case can be calculated by Fourier transforming the acquired signal. The complex MR images are obtained as

$$
\begin{gathered}
M_{c}(x, y)=M(x, y) \exp \left(j \gamma B t+j \theta_{c}\right) \\
M_{c j}(x, y)=M(x, y) \exp \left(j \gamma\left[B t+B_{j}(x, y) T_{c}\right]+j \theta_{c}\right)
\end{gathered}
$$

In these equations, $M_{c}(x, y)$ and $M_{c j}(x, y)$ are the complex MR images for nocurrent and current applied cases, respectively. Dividing the complex image for current applied case, by the complex image for no-current case, a resultant phase term is obtained as

$$
\frac{M_{c j}(x, y)}{M_{c}(x, y)}=\frac{M(x, y) \exp \left(j \gamma\left[B t+B_{j}(x, y) T_{c}\right]+j \theta_{c}\right)}{M(x, y) \exp \left(j \gamma B t+j \theta_{c}\right)}=\exp \left(j \gamma B_{j}(x, y) T_{c}\right)
$$

Finally, $B_{j}(x, y)$ is extracted by dividing the phase term in Equation (2.11) by gyromagnetic ratio, $\gamma$, and the duration of the applied current, $T_{c}$. 


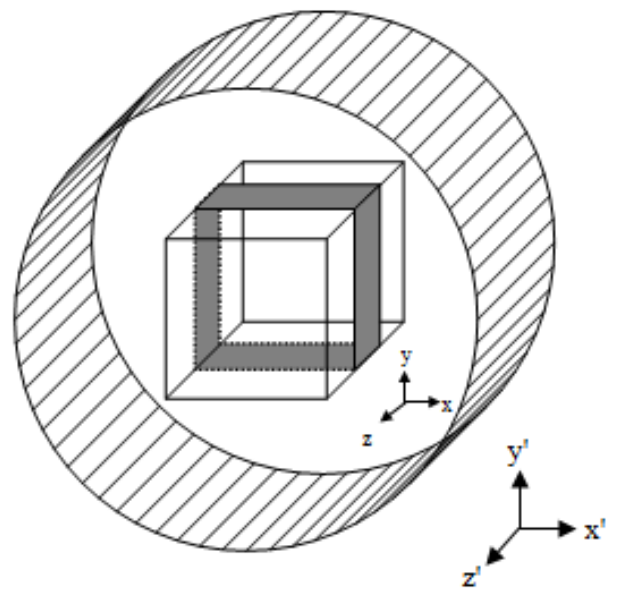

(a)

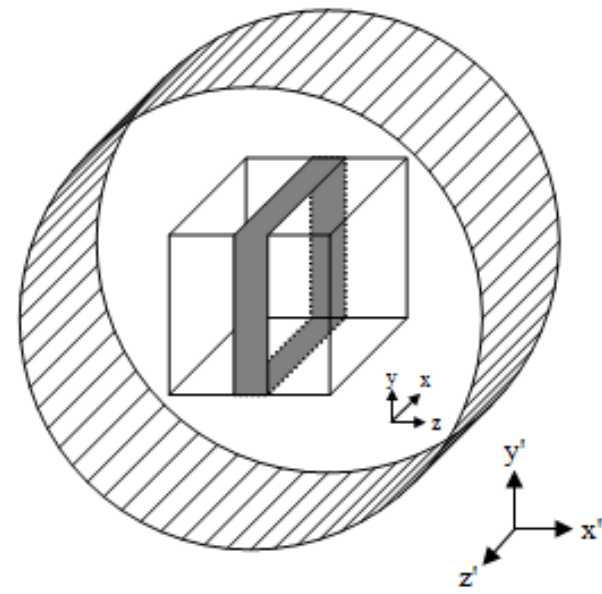

(b)

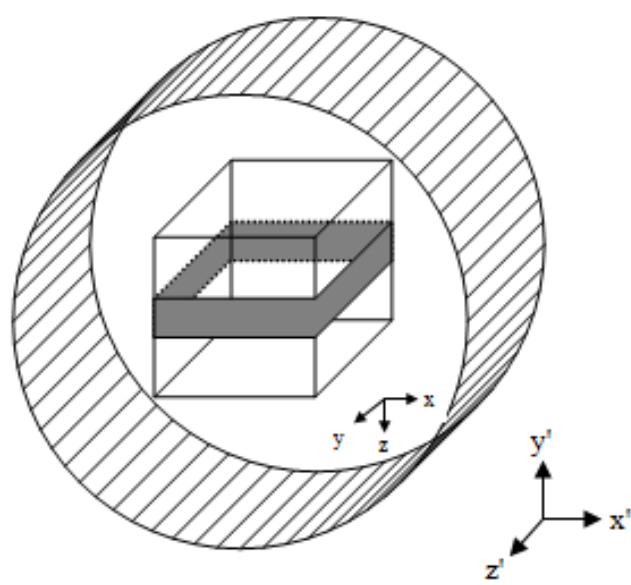

(c)

Figure 2.2 The placement of object inside MRI scanner for measuring all three components of magnetic flux density, a) measuring $B_{z}$, b) measuring $B_{x}$, c) measuring $B_{y}$

In classical MRCDI technique, all three components of magnetic flux density are required to reconstruct current density distribution. Therefore, the object must be rotated and the experiment must be repeated for three different placement of the object. The placement of the object inside MRI scanner for different experiments is given in Figure 2.2. The primed coordinate system $\left(x^{\prime}, y^{\prime}, z^{\prime}\right)$ is the coordinate system of the MRI system and $(x, y, z)$ is the coordinate system of the object. The main magnetic field of MRI system is in the $z^{\prime}$ direction. In Figure 2.2(a), the $\mathrm{z}$ axis of the object is aligned with $z^{\prime}$, hence, $B_{z}$ is measured. Similarly, in Figure 2.2(b) and Figure 2.2(c), $B_{x}$ and $B_{y}$ are measured, respectively. 
The relation between current density and magnetic flux density is defined by Maxwell's equation as

$$
\nabla \times \vec{B}=\mu_{0} \vec{J}
$$

By using the curl operator, Equation (2.12) can be written in open form as

$$
\vec{J}=\frac{1}{\mu_{0}}\left\{\left(\frac{\partial B_{z}}{\partial y}-\frac{\partial B_{y}}{\partial z}\right) \hat{a}_{x}+\left(\frac{\partial B_{x}}{\partial z}-\frac{\partial B_{z}}{\partial x}\right) \hat{a}_{y}+\left(\frac{\partial B_{y}}{\partial x}-\frac{\partial B_{x}}{\partial y}\right) \hat{a}_{z}\right\}
$$

In $2 \mathrm{D}$ applications, where current is confined in a plane, $\vec{J}$ is composed of two components, $J_{x}$ and $J_{y}$, which create a magnetic flux density only in z direction, $B_{z}$. Hence, $B_{x}$ and $B_{y}$ are equal to zero in $z_{0}$ plane and $\frac{\partial B_{y}}{\partial x}, \frac{\partial B_{x}}{\partial y}$ vanish on that plane. However, the derivatives of $B_{x}$ and $B_{y}$ with respect to $\mathrm{z}$ are not zero. In order to calculate these derivatives, $B_{x}$ and $B_{y}$ should be measured on $z_{0}+\Delta z$ and $z_{0}-\Delta z$ planes. So, their derivatives can be calculated as

$$
\begin{aligned}
& \left.\frac{\partial B_{x}}{\partial z}\right|_{z=z_{0}}=\frac{\left.B_{x}\right|_{z=z_{0}+\Delta z}-\left.B_{x}\right|_{z=z_{0}-\Delta z}}{2 \Delta z} \\
& \left.\frac{\partial B_{y}}{\partial z}\right|_{z=z_{0}}=\frac{\left.B_{y}\right|_{z=z_{0}+\Delta z}-\left.B_{y}\right|_{z=z_{0}-\Delta z}}{2 \Delta z}
\end{aligned}
$$

After calculating all the derivatives in Equation (2.13), the current density distribution can be reconstructed.

\subsection{Experimental Setup in 0.15 T METU-EE MRI System}

The extraction of magnetic flux density from MR images is mentioned in Section 2.4. In this section, experimental setup and procedure used in METU-EE MRI System will be given in detail.

The METU-EE MRI System is shown in Figure 2.3. The experimental setup consists of a main magnet, RF coil, current source, and the experimental phantom. 


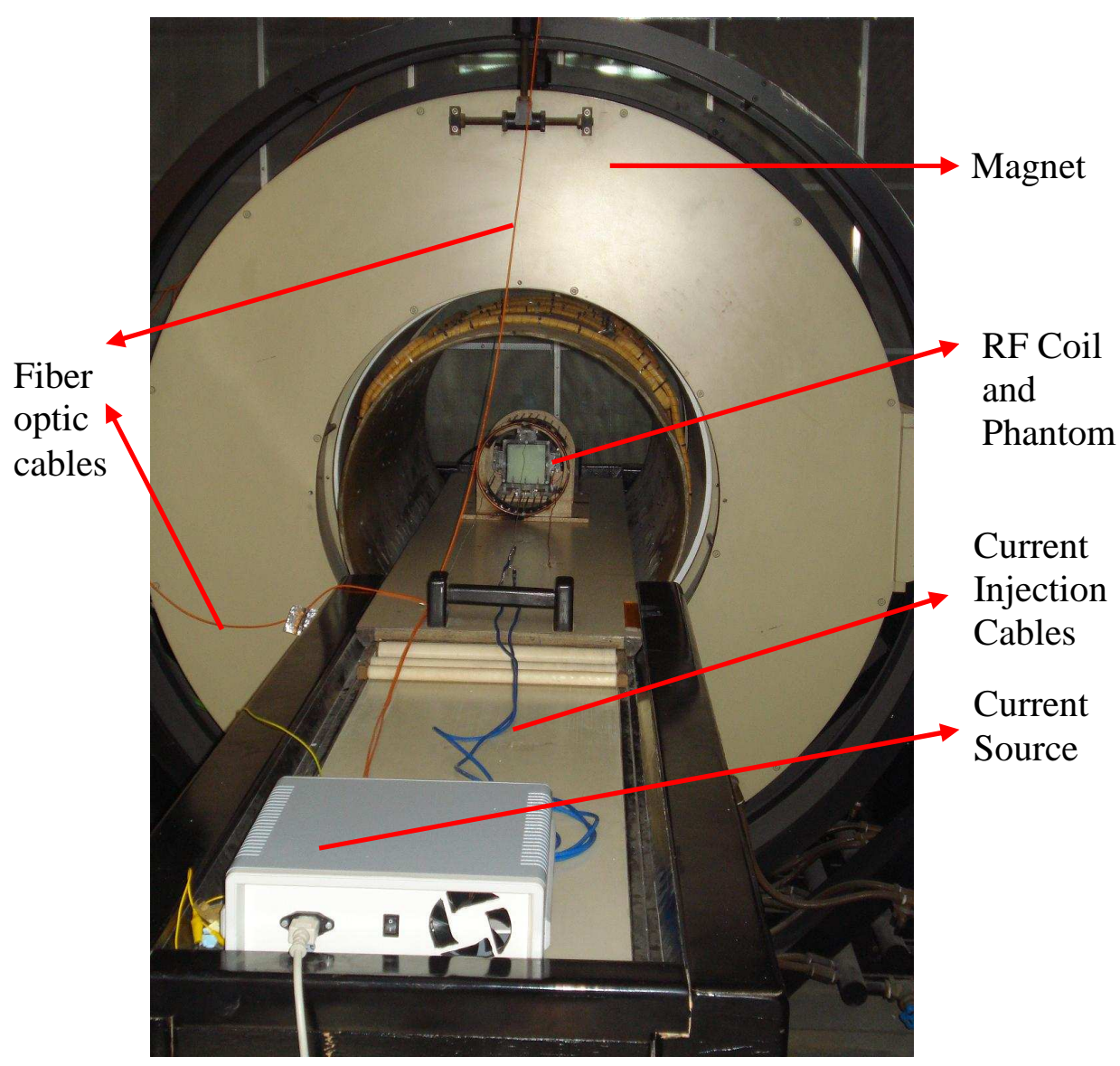

Figure 2.3 The experimental setup in METU MRI System.

The main magnet of this system is a resistive air core magnet with a bore diameter of $80 \mathrm{~cm}$. The resistance of the magnet is approximately $300 \mathrm{~m} \Omega$ and it is fed by a DC current source operating at $272 \mathrm{~A}$ to generate field strength of $0.15 \mathrm{~T} \mathrm{[27].}$

Designed RF coil shown in Figure 2.4 is a transceiver type of coil which can be used as both a transmitter and receiver coil. The RF coil is tuned before the experiments.

The current source is based on the design of Özbek [28] with some improvements. First of all, fiber optic transmitter and receiver units are added to the design. By these new units, the triggering pulse for current injection is transmitted from computer to the current source via fiber optic cables. The purpose of these units is to eliminate noise on triggering pulse. Detailed information about the current source design can be found in Değirmenci [29]. 


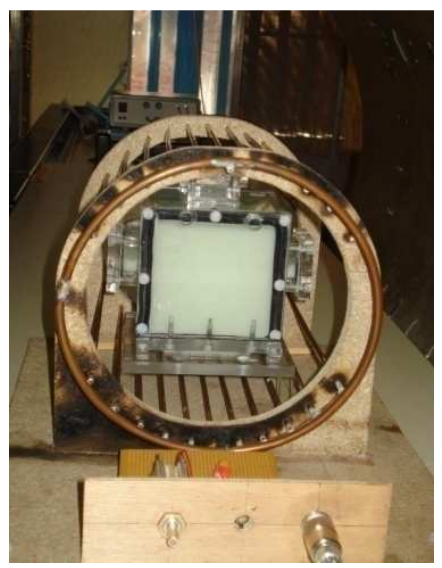

Figure 2.4 RF coil.

METU-EE MRI System is controlled by a software program which was developed by Özsüt [30]. In this software, desired pulse sequence can be designed as well as amplitude and duration of the applied current can be modified.

The experimental procedure for a MRCDI experiment in METU-EE MRI System is as follows:

- The pulse sequence is designed in the software.

- Amplitude and duration of the applied current is adjusted.

- Electrodes are connected to the experimental phantom.

- Current is applied and phase image is obtained.

- Current polarity is changed and phase image is obtained.

- The above steps are repeated for different current injection patterns.

After having phase images, magnetic flux density can be extracted as explained in Section 2.4. The screenshot of the software used in METU-EE MRI System for imaging $B_{z}$ is shown in Figure 2.5. 


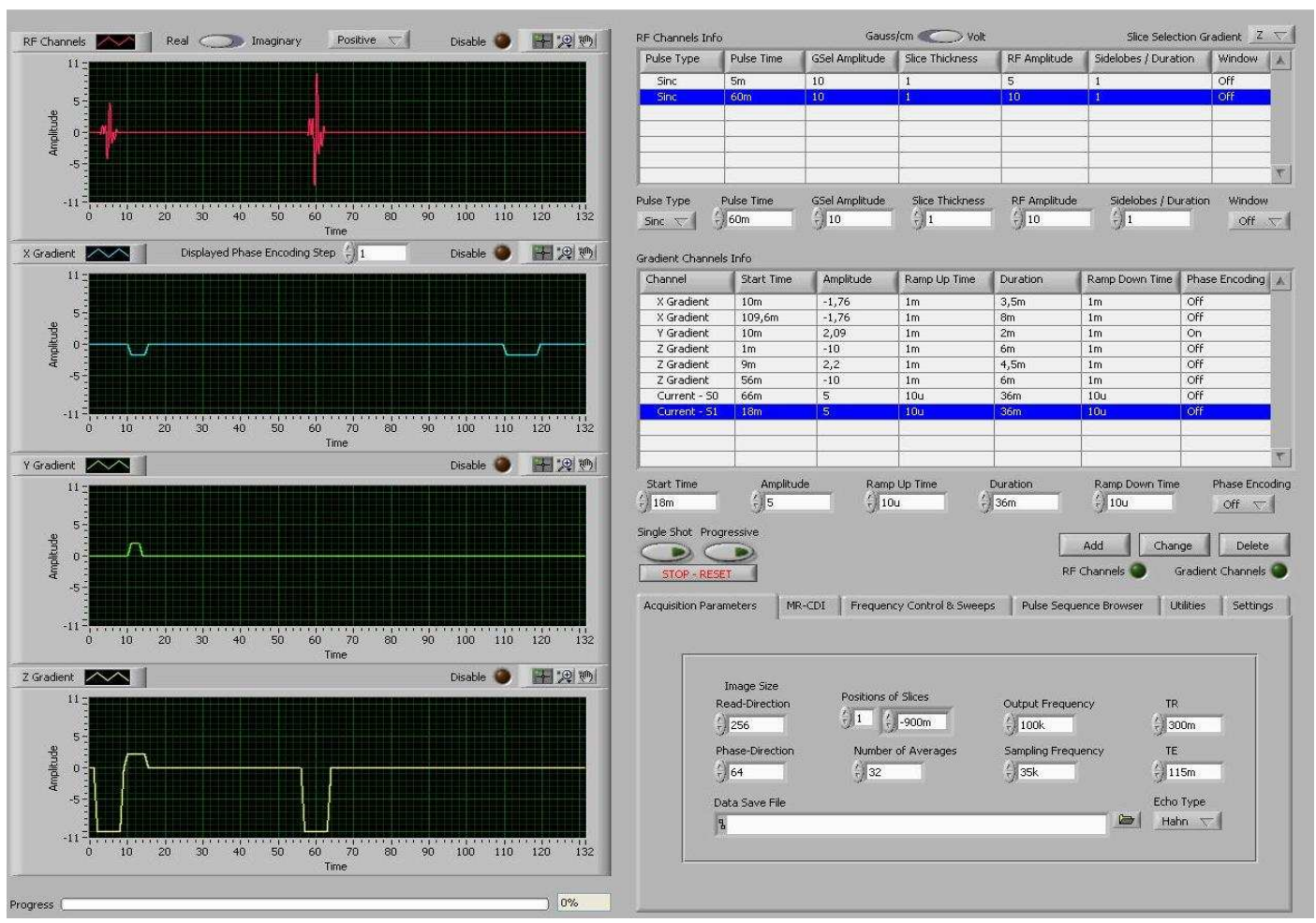

Figure 2.5 Screenshot of MRI software to image $B_{z}$.

In order to image $B_{x}$ and $B_{y}$, phantom orientation is changed depending on the magnetic flux density component to be imaged. Slice selection, frequency encoding and phase encoding gradients are adjusted accordingly. To image $B_{x}$, slice selection, frequency encoding and phase encoding gradients are in $\mathrm{x}, \mathrm{y}, \mathrm{z}$ directions, respectively. Similarly, slice selection, frequency encoding and phase encoding gradients are in $\mathrm{y}, \mathrm{z}, \mathrm{x}$ directions to image $B_{y}$.

It is stated in Section 2.4 that $B_{x}$ and $B_{y}$ should be measured on $z_{0}+\Delta z$ and $z_{0}-\Delta z$ planes to calculate the derivatives of $B_{x}$ and $B_{y}$ with respect to z. Therefore, in imaging $B_{x}$ and $B_{y}$, slice position is adjusted to $\Delta z$ above the center of imaging slice, $z_{0}$, and the phase image on that slice is obtained. Then, slice position is adjusted to $\Delta z$ below the center of imaging slice, $z_{0}$, and the phase image is obtained once again. Finally, current density distribution is calculated using Equation (2.13). The screenshots of the software used in METU-EE MRI System for imaging $B_{x}$ and $B_{y}$ are shown in Figure 2.6 and Figure 2.7, respectively. 


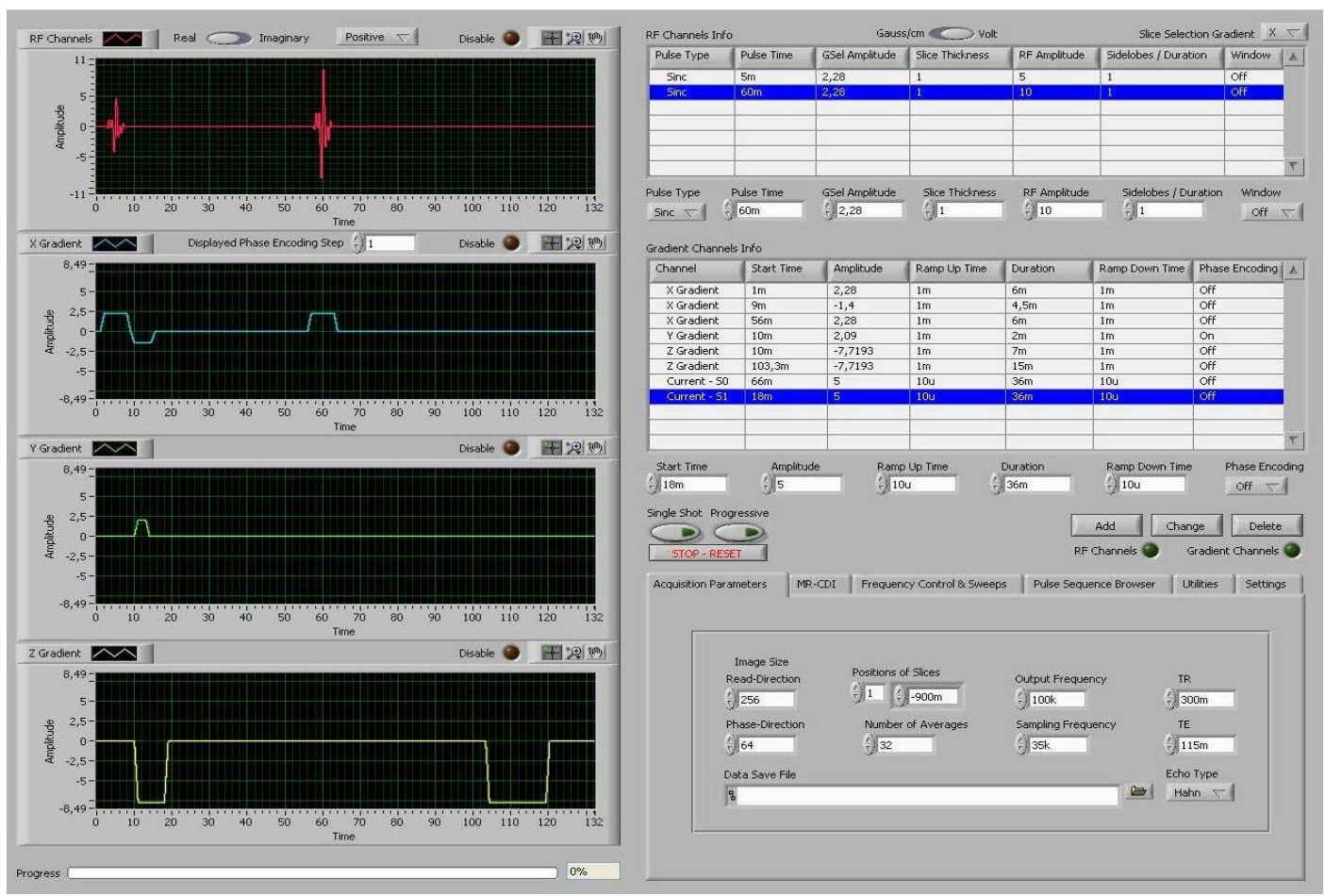

Figure 2.6 Screenshot of MRI software to image $B_{x}$.

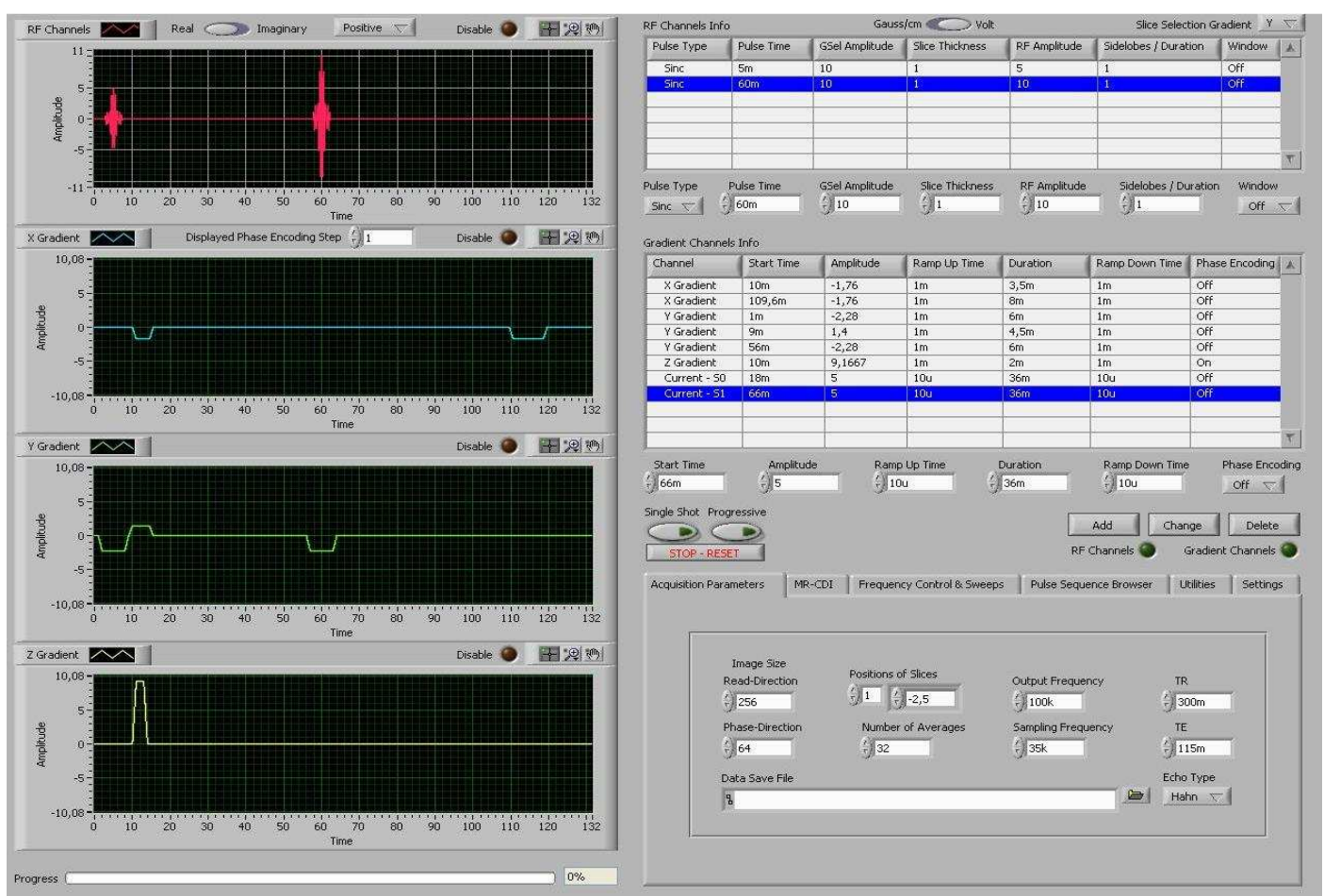

Figure 2.7 Screenshot of MRI software to image $B_{y}$. 


\section{CHAPTER 3}

\section{MAGNETIC RESONANCE CURRENT DENSITY IMAGING USING ONE COMPONENT OF MAGNETIC FLUX DENSITY}

\subsection{Introduction}

In this chapter, the inverse problem of Magnetic Resonance Current Density Imaging (MRCDI), which relates magnetic flux density to current density, will be investigated in detail. The theory and mathematical derivation of the proposed algorithm and the iterative FT-MRCDI algorithm will be also given in this chapter.

\subsection{Analysis of 2D MRCDI Problem}

In this section, 2D MRCDI inverse problem, which relates magnetic flux density to current density, will be analyzed in detail. First, the mathematical analysis of the problem will be given. Then, the sensitivity of magnetic flux density to current density as well as to the changes in conductivity will be investigated.

\subsubsection{Mathematical Analysis}

In MRCDI, the current density is calculated by measuring the magnetic flux density induced by current flow. The relation between quasi-static current density and magnetic flux density generated by this current is defined by Maxwell's equations as

$$
\vec{J}=\frac{\nabla \times \vec{B}}{\mu_{0}}
$$

where 
$\vec{J}$ : current density

$\vec{B}$ : magnetic flux density

$\mu_{0}$ : permeability of free space

$\vec{B}$ can be calculated by using Biot-Savart law as

$$
\vec{B}=\frac{\mu_{0}}{4 \pi} \int \frac{(\vec{J} d V) \times \vec{r}}{r^{3}}
$$

where

$r$ : distance between field and source points

$\vec{r}$ : vector from source to field point

$d V:$ differential volume element

The equations (3.1) and (3.2) are defined for 3D problems. In a 2D subject, $\Omega$, where currents flow in x-y plane, $\vec{J}$ has only two components, $J_{x}$ and $J_{y}$, which generate $\vec{B}$ only in z direction, $B_{z}$. The z-component of $\vec{B}, B_{z}$, is given as

$$
B_{z}(x, y)=\frac{\mu_{0}}{4 \pi} \int_{\Omega} \frac{J_{x}\left(x^{\prime}, y^{\prime}\right)\left(y-y^{\prime}\right)-\left(x-x^{\prime}\right) J_{y}\left(x^{\prime}, y^{\prime}\right)}{\left[\left(x-x^{\prime}\right)^{2}+\left(y-y^{\prime}\right)^{2}\right]^{\frac{3}{2}}} d x^{\prime} d y^{\prime}
$$

in $\mathrm{z}=0$ plane. The equation (3.3) is valid for the cases where the subject is infinitely long along the longitudinal direction. Now, consider a circular slice of a conductor region having a conductivity distribution $\sigma$, radius $a$ and thickness $d$ as shown in Figure 3.1. The circular geometry is chosen in order to simplify analytical derivations.

Let $\sigma$ be the conductivity distribution as

$$
\sigma=\left\{\begin{array}{l}
\sigma_{1}, \text { for } 0<r<b \\
\sigma_{2}, \text { for } \mathrm{b}<r<a
\end{array}\right.
$$


The electrodes having width $\delta$ and height $d$ are placed at the sides of the conductor region at the angular positions $\theta_{1}$ and $\theta_{2}$.

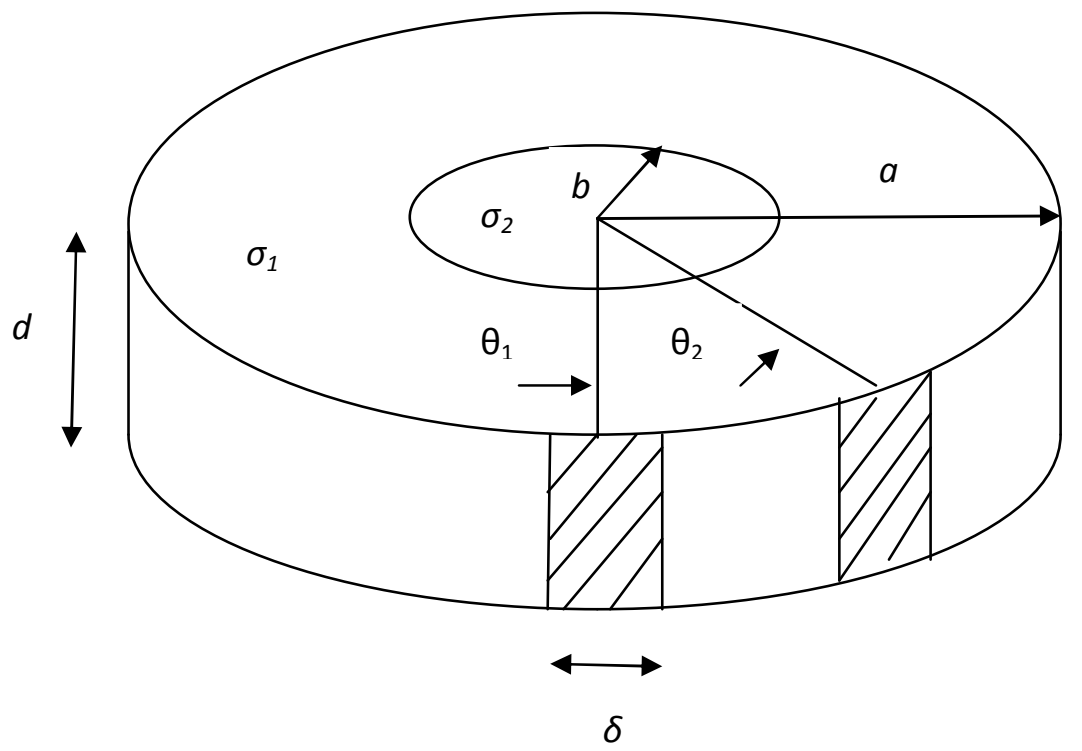

Figure 3.1 The geometry of the conductor region used for analytical derivations.

The current applied on the boundaries can be expressed by an infinite summation of circular harmonics as

$$
j(\varphi)=\sum_{n=1}^{\infty} C_{n} \cos (n \varphi)+S_{n} \sin (n \varphi)
$$

where

$$
C_{n}=\frac{1}{\pi} \int_{0}^{2 \pi} j(\varphi) \cos (n \varphi) d \varphi
$$

and

$$
S_{n}=\frac{1}{\pi} \int_{0}^{2 \pi} j(\varphi) \sin (n \varphi) d \varphi
$$

Assume that a current, $I$, is applied from the electrodes as 


$$
j(\varphi)=\left\{\begin{array}{lc}
\frac{I}{d \delta} & \theta_{1}-\frac{\beta}{2} \leq \varphi \leq \theta_{1}+\frac{\beta}{2} \\
-\frac{I}{d \delta} & \theta_{2}-\frac{\beta}{2} \leq \varphi \leq \theta_{2}+\frac{\beta}{2}
\end{array}\right.
$$

where $\beta=\delta / a$ is the angle covered by an electrode. Then, the voltage distribution inside the conductor region can be expressed by Laplace's equation. Note that, the voltage distribution, $\Phi(r, \varphi, z)$, is invariant in z direction. The solution of Laplace's equation can be represented by an infinite sum of circular harmonics as

$$
\Phi(r, \varphi)=\left\{\begin{array}{l}
\Phi_{1}(r, \varphi) \text { if } 0 \leq r \leq b \\
\Phi_{2}(r, \varphi) \text { if } b \leq r \leq a
\end{array}\right.
$$

where

$$
\Phi_{1}(r, \varphi)=\sum_{n=1}^{\infty} r^{n}\left[A_{n} \cos (n \varphi)+B_{n} \sin (n \varphi)\right]
$$

and

$$
\Phi_{2}(r, \varphi)=\left\{\begin{array}{l}
\sum_{n=1}^{\infty} r^{n}\left[E_{n} \cos (n \varphi)+F_{n} \sin (n \varphi)\right] \\
+\sum_{n=1}^{\infty} r^{-n}\left[G_{n} \cos (n \varphi)+H_{n} \sin (n \varphi)\right]
\end{array}\right\}
$$

Boundary conditions are given as

$$
\begin{gathered}
\Phi_{1}(b, \varphi)=\Phi_{2}(b, \varphi) \\
-\left.\sigma_{1} \frac{\partial \Phi_{1}(r, \varphi)}{\partial r}\right|_{r=b}=-\left.\sigma_{2} \frac{\partial \Phi_{2}(r, \varphi)}{\partial r}\right|_{r=b} \\
-\left.\sigma_{2} \frac{\partial \Phi_{2}(r, \varphi)}{\partial r}\right|_{r=a}=j(\varphi)
\end{gathered}
$$

The coefficients $C_{n}$ and $S_{n}$ can be calculated by evaluating the integrals in Equations (3.6) and (3.7). Also, the coefficients $A_{n}, B_{n}, E_{n}, F_{n}, G_{n}, H_{n}$ in Equations (3.10) and (3.11) can be calculated by solving these equations with boundary conditions in 
Equations (3.12), (3.13) and (3.14). The coefficients are found as:

$$
\begin{gathered}
C_{n}=\frac{I}{n \pi d \delta}\left[\left.\sin (n \varphi)\right|_{\theta_{1}-\frac{\beta}{2}} ^{\theta_{1}+\frac{\beta}{2}}-\left.\sin (n \varphi)\right|_{\theta_{2}-\frac{\beta}{2}} ^{\theta_{2}+\frac{\beta}{2}}\right] \\
S_{n}=-\frac{I}{n \pi d \delta}\left[\left.\cos (n \varphi)\right|_{\theta_{1}-\frac{\beta}{2}} ^{\theta_{1}+\frac{\beta}{2}}-\left.\cos (n \varphi)\right|_{\theta_{2}-\frac{\beta}{2}} ^{\theta_{2}+\frac{\beta}{2}}\right] \\
A_{n}=\frac{-2 C_{n} b^{-2 n}}{n a^{n-1}\left[\left(\sigma_{1}+\sigma_{2}\right) b^{-2 n}-\left(\sigma_{2}-\sigma_{1}\right) a^{-2 n}\right]} \\
B_{n}=\frac{-2 S_{n} b^{-2 n}}{n a^{n-1}\left[\left(\sigma_{1}+\sigma_{2}\right) b^{-2 n}-\left(\sigma_{2}-\sigma_{1}\right) a^{-2 n}\right]} \\
E_{n}=\frac{-\left(\sigma_{1}+\sigma_{2}\right) C_{n} b^{-2 n}}{n a^{n-1}\left[\left(\sigma_{1}+\sigma_{2}\right) b^{-2 n}-\left(\sigma_{2}-\sigma_{1}\right) a^{-2 n}\right] \sigma_{2}} \\
G_{n}=\frac{-\left(\sigma_{2}-\sigma_{1}\right) S_{n}}{n a^{n-1}\left[\left(\sigma_{1}+\sigma_{2}\right) b^{-2 n}-\left(\sigma_{2}-\sigma_{1}\right) a^{-2 n}\right] \sigma_{2}} \\
-\left(\sigma_{1}+\sigma_{2}\right) S_{n} b^{-2 n}
\end{gathered}
$$

The details of the solution are given in appendix A. After finding the voltage distribution inside the conductor region, the current density distribution can be evaluated. It is known that

$$
\vec{J}=-\sigma \nabla \Phi
$$

and

$$
\nabla=\hat{a}_{r} \frac{\partial}{\partial r}+\hat{a}_{\varphi} \frac{1}{r} \frac{\partial}{\partial \varphi}
$$


Hence, the current density distribution is found as follows

$$
\begin{gathered}
J_{r}(r, \varphi)=\left\{\begin{array}{l}
-\sum_{n=1}^{\infty} \sigma_{1} n r^{n-1}\left[A_{n} \cos (n \varphi)+B_{n} \sin (n \varphi)\right] \text { if } 0 \leq r \leq b \\
-\sum_{n=1}^{\infty} \sigma_{2} n r^{n-1}\left[E_{n} \cos (n \varphi)+F_{n} \sin (n \varphi)\right] \\
+\sum_{n=1}^{\infty} \sigma_{2} n r^{-n-1}\left[G_{n} \cos (n \varphi)+H_{n} \sin (n \varphi)\right]
\end{array}\right\} \text { if } b \leq r \leq a
\end{gathered}
$$

Since the current density distribution is expressed in cylindrical coordinates, the Biot-Savart equation should also be written in cylindrical coordinates to obtain a relation between magnetic flux density and current density distribution. As it was mentioned, Equation (3.3) is valid for the cases where the conductor region is infinitely long along the longitudinal direction. However, in this case, the conductor region has a finite thickness, $d$. If it is assumed that the thickness of the conductor region is too small compared to its radius, the Equation (3.3) becomes

$$
B_{z}(x, y, z)=\frac{\mu_{0} d}{4 \pi} \int_{\Omega} \frac{J_{x}\left(x^{\prime}, y^{\prime}\right)\left(y-y^{\prime}\right)-\left(x-x^{\prime}\right) J_{y}\left(x^{\prime}, y^{\prime}\right)}{\left[\left(x-x^{\prime}\right)^{2}+\left(y-y^{\prime}\right)^{2}+z^{2}\right]^{\frac{3}{2}}} d x^{\prime} d y^{\prime}
$$

$J_{x}$ and $J_{y}$ in Equation (3.27) can be transformed into cylindrical coordinates by using

$$
\begin{array}{ll}
J_{x}\left(x^{\prime}, y^{\prime}\right)=J_{r}\left(r^{\prime}, \varphi^{\prime}\right) \cos \varphi^{\prime}-J_{\varphi}\left(r^{\prime}, \varphi^{\prime}\right) \sin \varphi^{\prime} \\
J_{y}\left(x^{\prime}, y^{\prime}\right)=J_{r}\left(r^{\prime}, \varphi^{\prime}\right) \sin \varphi^{\prime}+J_{\varphi}\left(r^{\prime}, \varphi^{\prime}\right) \cos \varphi^{\prime} \\
x=r \cos \varphi & x^{\prime}=r^{\prime} \cos \varphi^{\prime} \\
y=r \sin \varphi & y^{\prime}=r^{\prime} \sin \varphi^{\prime}
\end{array}
$$

Then, Equation (3.27) becomes 


$$
B_{z}(r, \varphi, z)=\left\{\begin{array}{c}
\frac{\mu_{0} d}{4 \pi} \int_{\Omega} \frac{\left[J_{r}\left(r^{\prime}, \varphi^{\prime}\right) \cos \varphi^{\prime}-J_{\varphi}\left(r^{\prime}, \varphi^{\prime}\right) \sin \varphi^{\prime}\right]\left(r \sin \varphi-r^{\prime} \sin \varphi^{\prime}\right)}{\left[\left(r \cos \varphi-r^{\prime} \cos \varphi^{\prime}\right)^{2}+\left(r \sin \varphi-r^{\prime} \sin \varphi^{\prime}\right)^{2}+z^{2}\right]^{\frac{3}{2}}} d s^{\prime} \\
-\frac{\mu_{0} d}{4 \pi} \int_{\Omega} \frac{\left[J_{r}\left(r^{\prime}, \varphi^{\prime}\right) \sin \varphi^{\prime}+J_{\varphi}\left(r^{\prime}, \varphi^{\prime}\right) \cos \varphi^{\prime}\right]\left(r \cos \varphi-r^{\prime} \cos \varphi^{\prime}\right)}{\left[\left(r \cos \varphi-r^{\prime} \cos \varphi^{\prime}\right)^{2}+\left(r \sin \varphi-r^{\prime} \sin \varphi^{\prime}\right)^{2}+z^{2}\right]^{\frac{3}{2}}} d s^{\prime}
\end{array}\right\}
$$

Reorganizing the terms in Equation (3.29) gives

$$
B_{z}(r, \varphi, z)=\frac{\mu_{0} d}{4 \pi} \int_{\Omega} \frac{J_{r}\left(r^{\prime}, \varphi^{\prime}\right) r \sin \left(\varphi-\varphi^{\prime}\right)+J_{\varphi}\left(r^{\prime}, \varphi^{\prime}\right)\left[r^{\prime}-r \cos \left(\varphi-\varphi^{\prime}\right)\right]}{\left[r^{2}+r^{\prime 2}-2 r r^{\prime} \cos \left(\varphi-\varphi^{\prime}\right)+z^{2}\right]^{\frac{3}{2}}} d s^{\prime}
$$

Calculating $B_{z}, J_{r}$ and $J_{\varphi}$ analytically makes it possible to obtain the sensitivity of $B_{z}$, $J_{r}, J_{\varphi}$ to the changes in conductivity and the radius of the concentric inhomogeneity.

\subsubsection{Sensitivity Plots for $B_{z}, J_{x}$ and $J_{y}$}

In Section 3.2.1, the relation between $B_{z}, J_{x}, J_{y}$ and $\sigma$ is obtained analytically. Now, the sensitivity of $B_{z}, J_{x}, J_{y}$ to the changes in both conductivity and the radius of the concentric inhomogeneity will be analyzed. For this purpose, the geometry used for analytical derivations shown in Figure 3.1 is implemented in MATLAB.

In the previous subsection, the current applied on the boundaries was expressed by an infinite summation of circular harmonics as in Equation (3.5). However, infinite summation is not possible in applications. Therefore, Equation (3.5) is modified as a finite summation:

$$
j(\varphi)=\sum_{n=1}^{N} C_{n} \cos (n \varphi)+S_{n} \sin (n \varphi)
$$

Here, $N$ is increased until the change in the applied current, $j(\varphi)$, between successive $N$ values reduces below a predetermined value.

Another important parameter is the electrode size and its placement on the simulation model. The electrodes are placed on the top and the bottom as well as on the left and right side of the conductor region. However, only horizontal current injection pattern is used in analytical solution because of the symmetry in the geometry of the model. 
The electrode size is chosen one-tenth of the perimeter of the conductor region. The model prepared in MATLAB is shown in Figure 3.2. In this figure, $\sigma_{1}$ is the conductivity of the concentric inhomogeneity, $\sigma_{2}$ is the conductivity of the background, $b$ is the radius of the concentric inhomogeneity and $a$ is the radius of the conductor region.

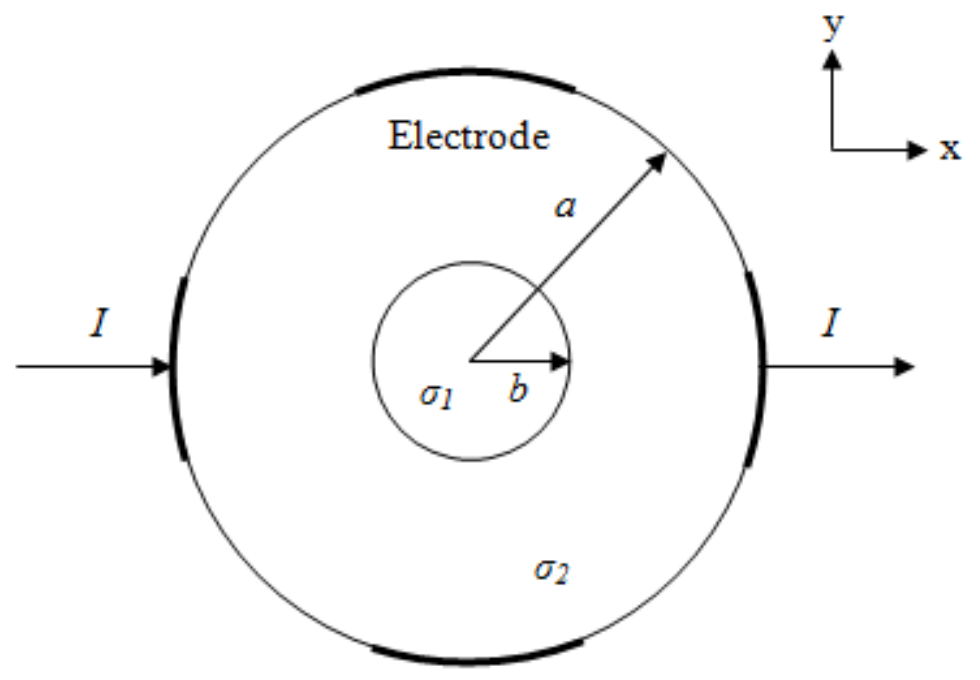

Figure 3.2 The geometry of the conductor region prepared in MATLAB.

Sample analytical solution result obtained from the prepared model is shown in Figure 3.3. It is important to note that the magnetic flux density outside the conductor region is zero in Figure 3.3d since the area outside the conductor region is masked. The reason of masking is that the magnetic flux density outside the conductor region can't be measured by using an MRI scanner in real applications.

To analyze the relation between $B_{z}, J_{x}$ and $J_{y}$, it is convenient to define the sensitivity as

$$
\begin{aligned}
& S B_{z} J_{x}=\frac{\left\|B_{z}-B_{z}^{\text {uniform }}\right\|}{\left\|B_{z}^{\text {uniform }}\right\|} \cdot \frac{\left\|J_{x}^{\text {uniform }}\right\|}{\left\|J_{x}-J_{x}^{\text {uniform }}\right\|} \\
& S B_{z} J_{y}=\frac{\left\|B_{z}-B_{z}^{\text {uniform }}\right\|}{\left\|B_{z}^{\text {uniform }}\right\|} \cdot \frac{\left\|J_{y}^{\text {uniform }}\right\|}{\left\|J_{y}-J_{y}^{\text {uniform }}\right\|}
\end{aligned}
$$

Here, $\|$.$\| is the Frobenius norm which is defined as$ 


$$
\|A\|=\sqrt{\sum_{i=1}^{m} \sum_{j=1}^{n}\left|a_{i j}\right|^{2}}
$$

$S B_{z} J_{x}$ is the change of $B_{z}$ from $B_{z}^{\text {uniform }}$ for a change of $J_{x}$ from $J_{x}^{\text {uniform }}$. Similarly, $S B_{z} J_{y}$ is the change of $B_{z}$ from $B_{z}^{\text {uniform }}$ for a change of $J_{y}$ from $J_{y}^{\text {uniform }}$. Here, $J_{x}^{\text {uniform }}$ and $J_{y}{ }^{\text {uniform }}$ are the currents for uniform conductivity distribution and $B_{z}^{\text {uniform }}$ is generated by using uniform currents.

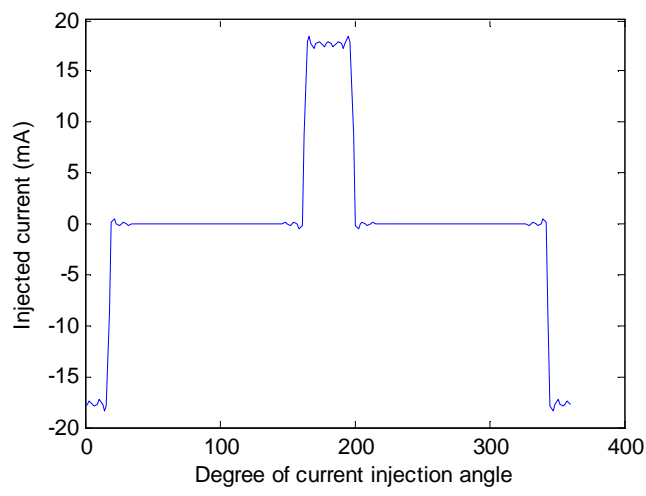

(a)

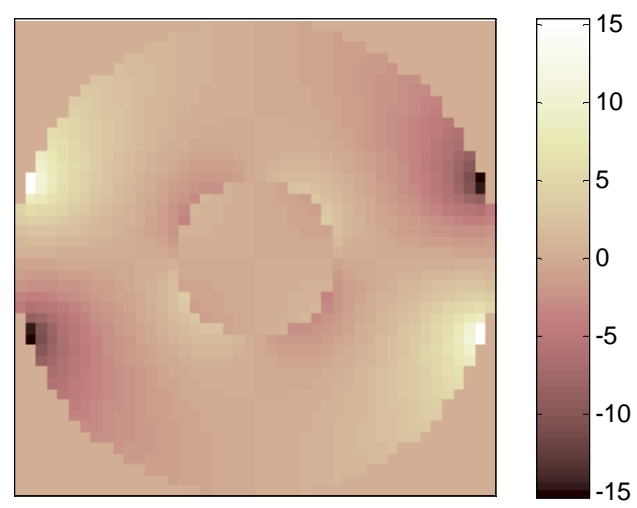

(c)

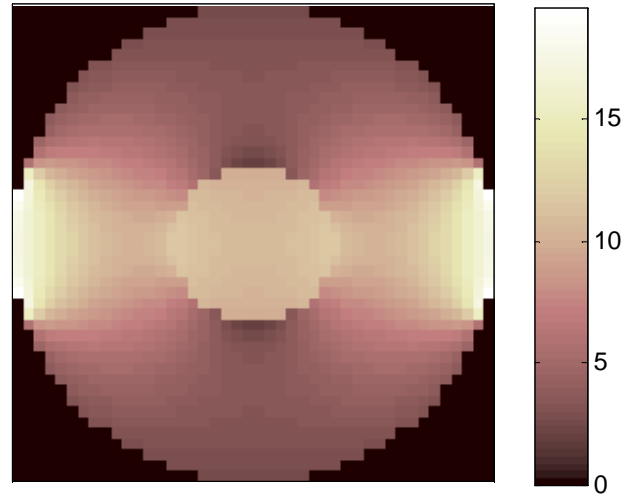

(b)

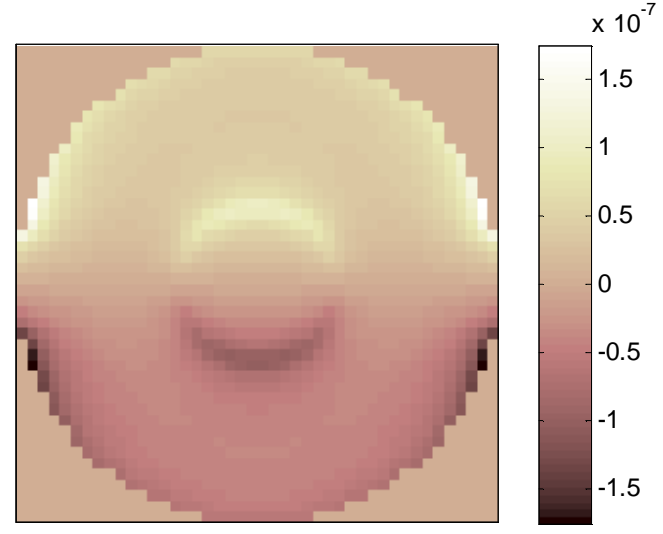

(d)

Figure 3.3 Analytical solution results of the model for $\sigma_{1}=1 \mathrm{~S} / \mathrm{m}, \sigma_{2}=0.2 \mathrm{~S} / \mathrm{m}, \mathrm{b}=3$ $\mathrm{cm}, \mathrm{a}=9 \mathrm{~cm}$ and $20 \mathrm{~mA}$ current is applied from the electrode on the left and sunk from the electrode on the right, a) The amplitude of the applied current, b) $J_{x}\left(\mathrm{~A} / \mathrm{m}^{2}\right)$ image for vertical current injection, c) $J_{y}\left(\mathrm{~A} / \mathrm{m}^{2}\right)$ image for vertical current injection, d) $B_{z}(\mathrm{~T})$ image for vertical current injection.

Some new terms are defined in order to simplify the variables used in the plots. The first term is sigma_ratio which is the ratio of conductivity of the inhomogeneity to conductivity of the background. The other one is radius_ratio which is defined as the 
ratio of radius of the inhomogeneity to radius of the conductor region. In this study, the radius of the conductor region, $a$, is set to $9 \mathrm{~cm}$ and the conductivity of background, $\sigma_{2}$, is set to $1 \mathrm{~S} / \mathrm{m}$.

Before starting the analysis of $S B_{z} J_{x}$ and $S B_{z} J_{y}$, it will be helpful to see the behavior of $\left\|J_{x}-J_{x}^{\text {uniform }}\right\|,\left\|J_{y}-J_{y}^{\text {uniform }}\right\|$ and $\left\|B_{z}-B_{z}^{\text {uniform }}\right\|$ with respect to sigma_ratio and radius_ratio. These plots are given in Figure 3.4, Figure 3.5, Figure 3.6, Figure 3.7, Figure 3.8 and Figure 3.9.

In Figure 3.4, $\left\|J_{x}-J_{x}^{\text {uniform }}\right\|$ increases as the conductivity of the inhomogeneity diverges from the conductivity of the background. This situation is expected since more current flows through the inhomogeneity in conductive case and more current flows around the inhomogeneity in insulating case. However, its value decreases after the radius_ratio is equal to 0.65 . This is because of the fact that the radius of the inhomogeneity becomes comparable to the radius of the conductor region and the current density distribution in the inhomogeneity becomes similar to the current density distribution in uniform conductivity case. The same situations can be seen in Figure 3.6 and Figure 3.8. Figure 3.8 also gives valuable information about the distinguishability of the inhomogeneities placed on the background. If the precision of the magnetic flux density measurements is defined as $\varepsilon$, then it can be said that an inhomogeneity placed on the center of the conductor region can be distinguished if

$$
\left\|B_{z}-B_{z}^{\text {uniform }}\right\|>\varepsilon
$$

It can be concluded from Figure 3.8 that the distinguishability of an inhomogeneity depends on its radius and its conductivity. 


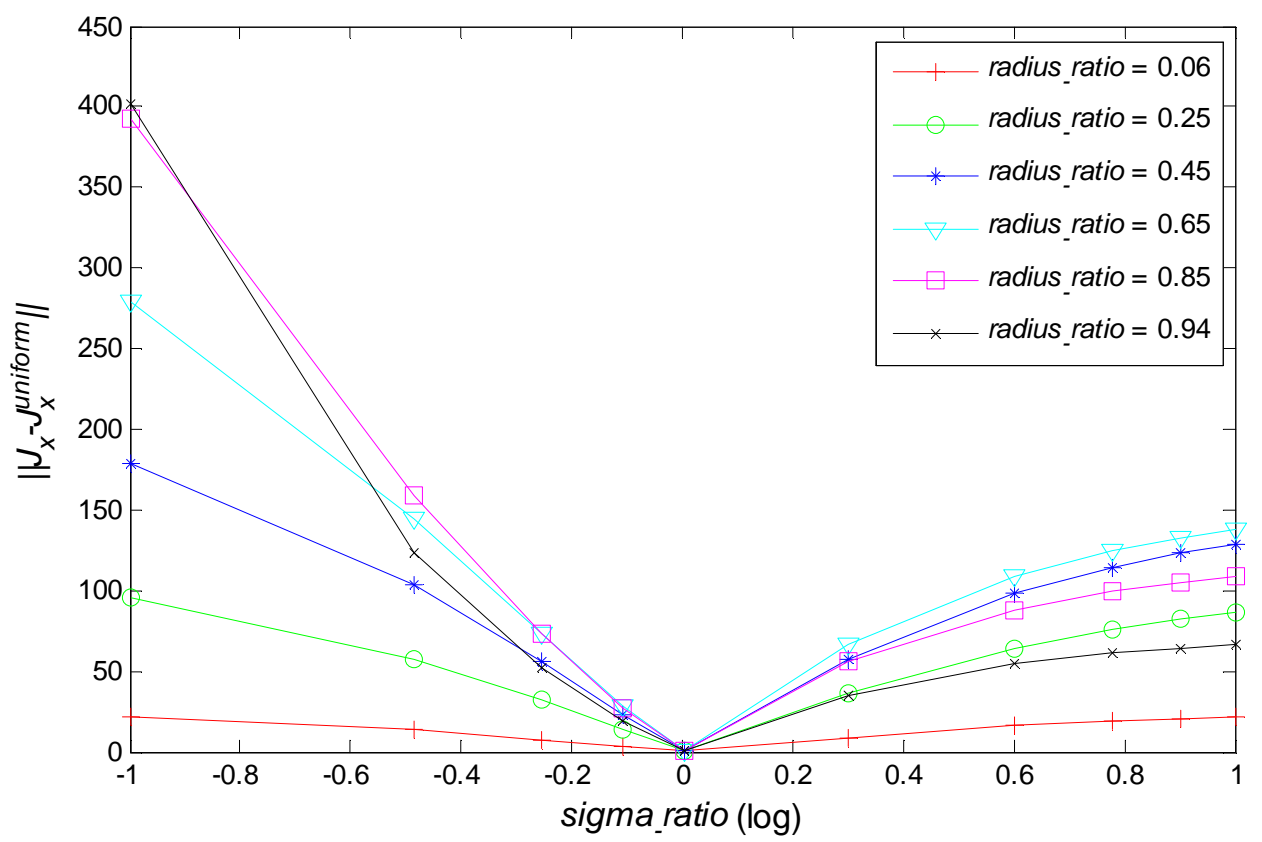

Figure $3.4\left\|J_{x}-J_{x}^{\text {uniform }}\right\|$ vs. sigma_ratio plot for various radius_ratio values.

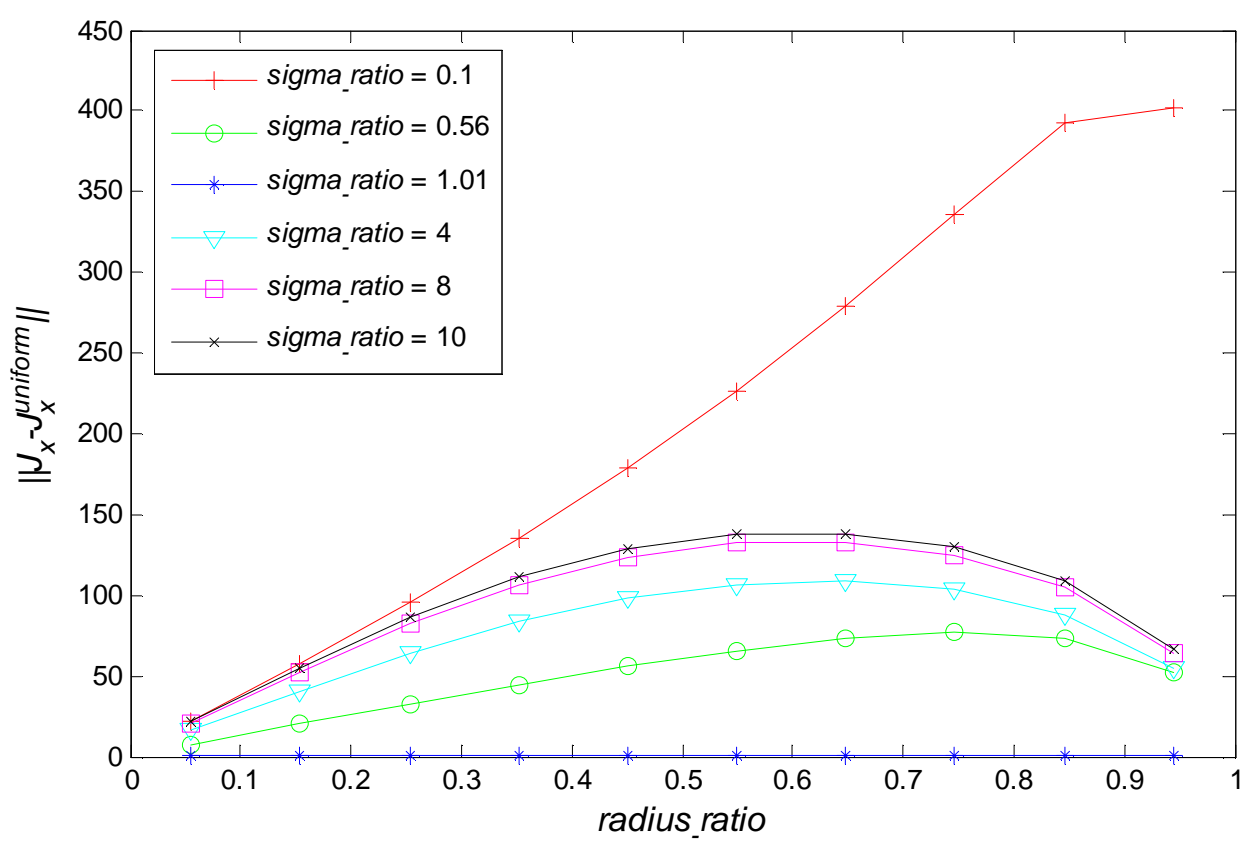

Figure $3.5\left\|J_{x}-J_{x}^{\text {uniform }}\right\|$ vs. radius_ratio plot for various sigma_ratio values. 


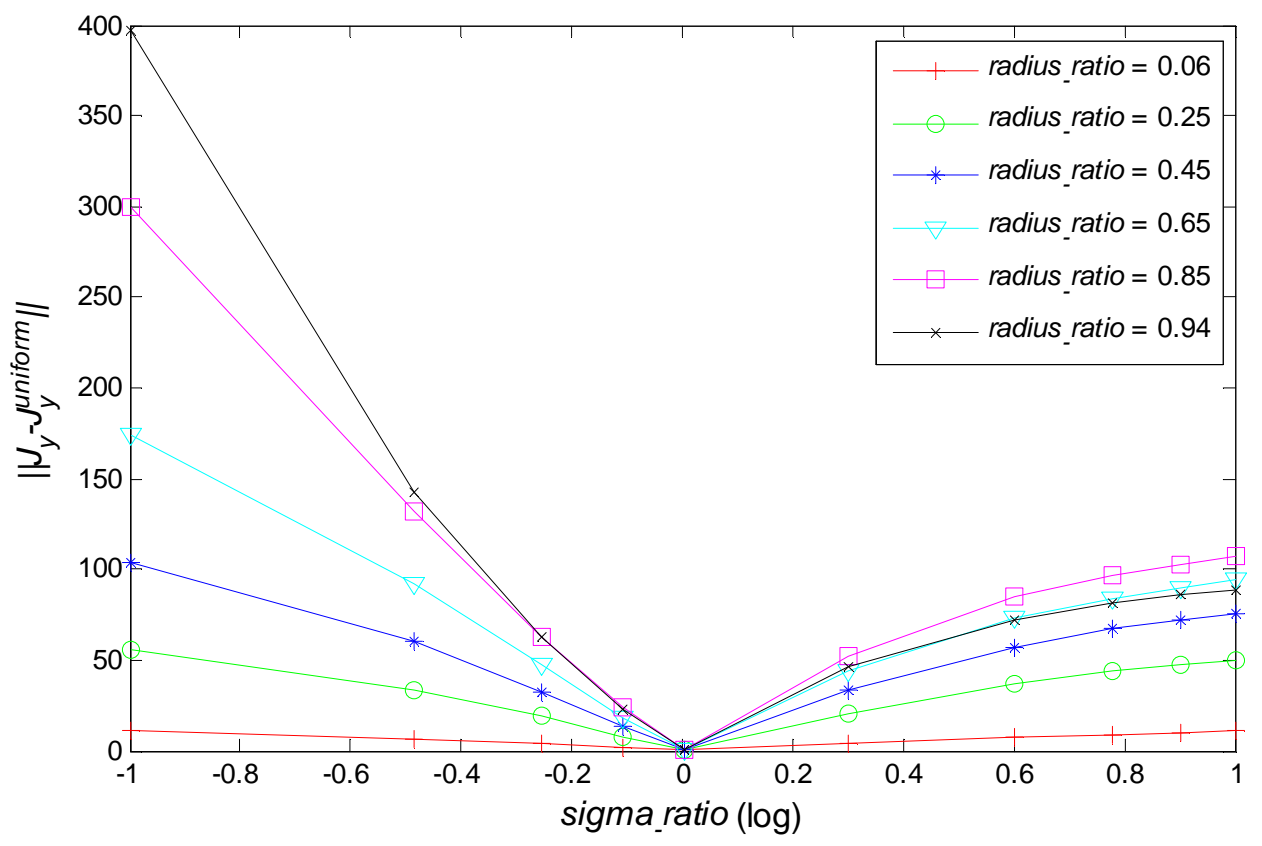

Figure $3.6\left\|J_{y}-J_{y}^{\text {uniform }}\right\|$ vs. sigma_ratio plot for various radius_ratio values.

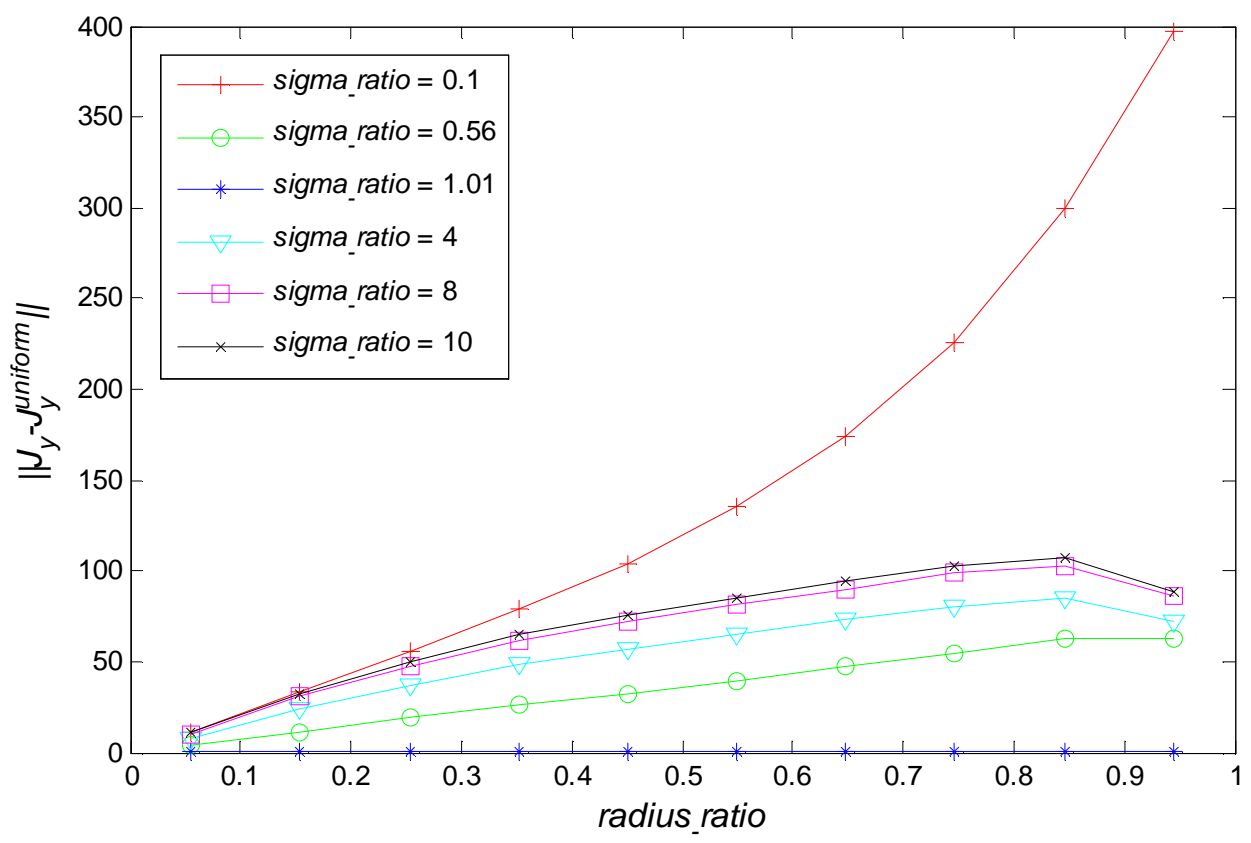

Figure $3.7\left\|J_{y}-J_{y}^{\text {uniform }}\right\|$ vs. radius_ratio plot for various sigma_ratio values. 


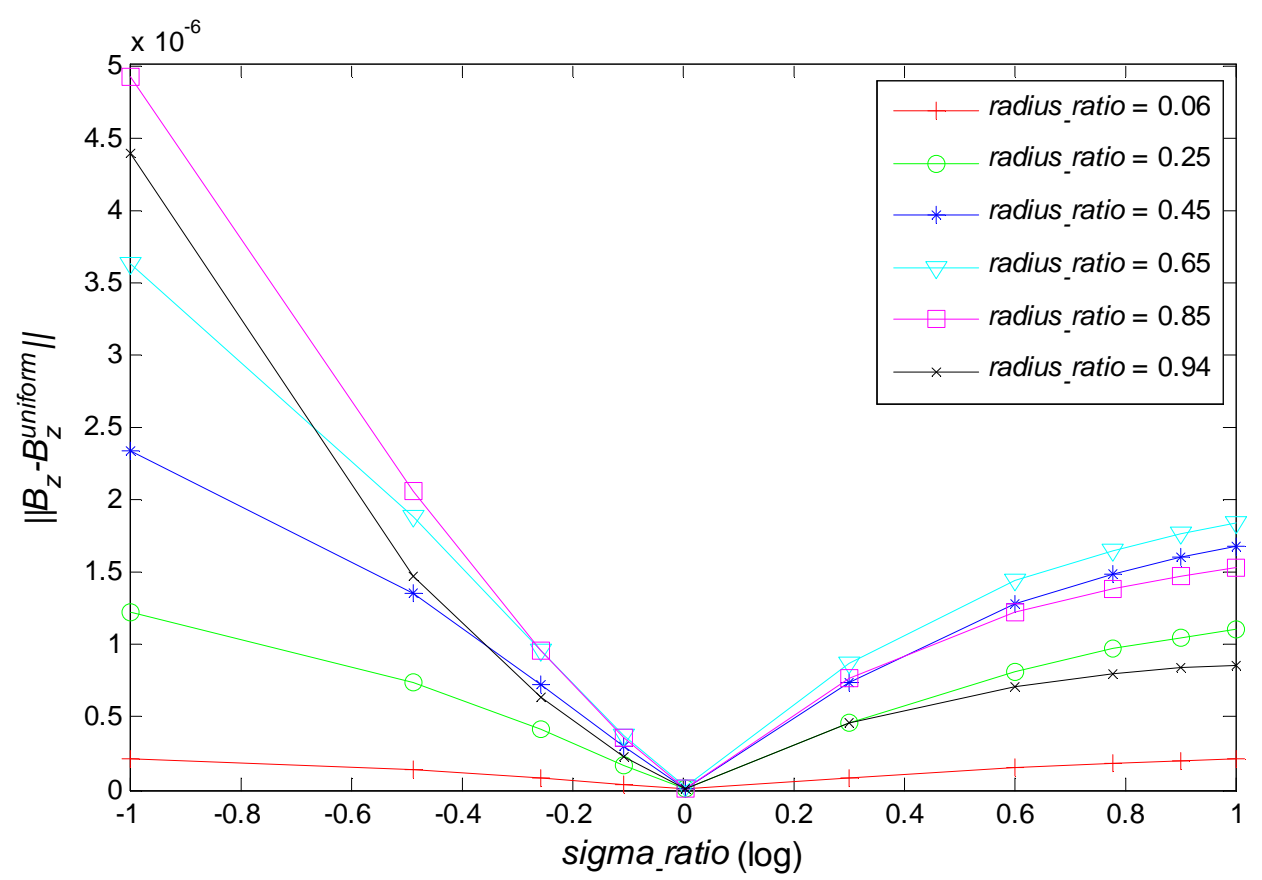

Figure $3.8\left\|B_{z}-B_{z}^{\text {uniform }}\right\|$ vs. sigma_ratio plot for various radius_ratio values.

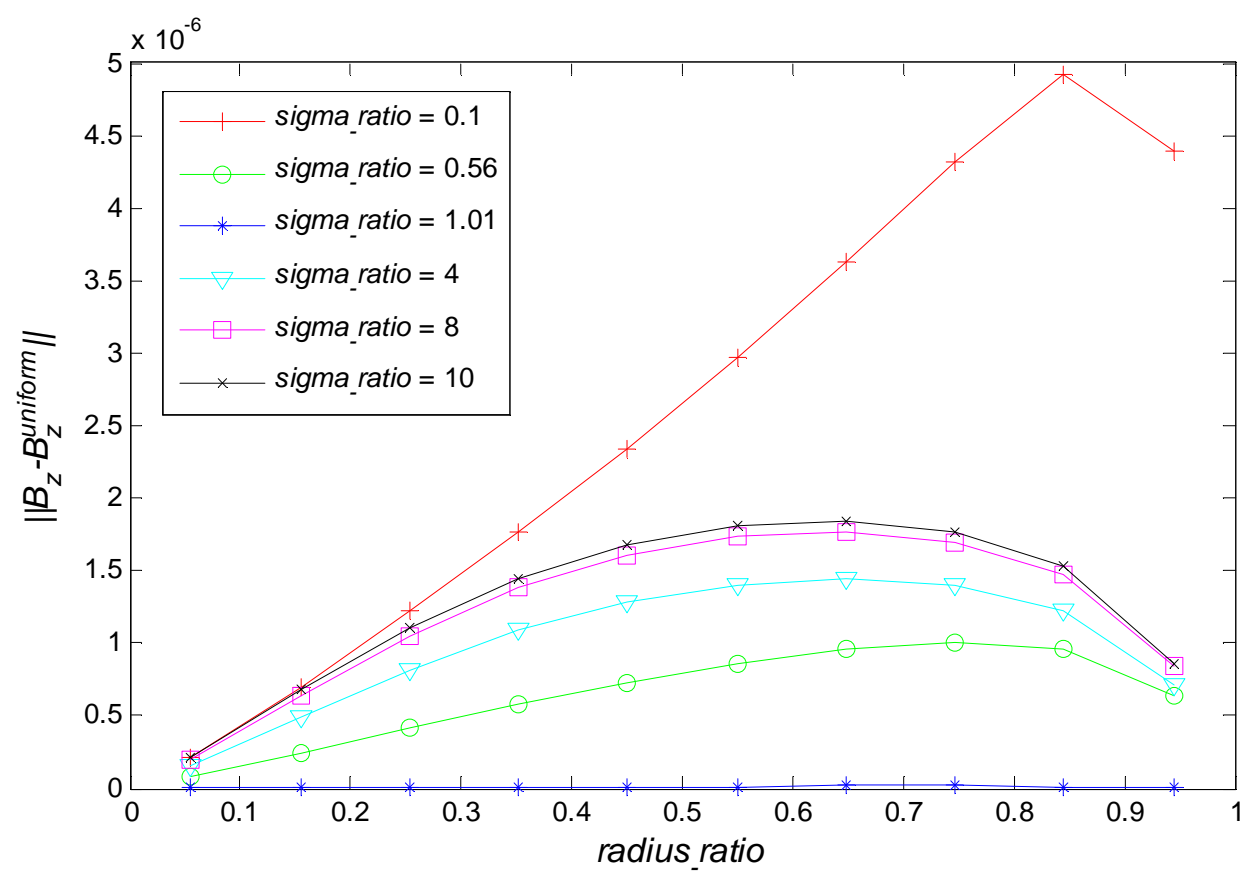

Figure $3.9\left\|B_{z}-B_{z}^{\text {uniform }}\right\|$ vs. radius_ratio plot for various sigma_ratio values. 
The behaviors of $\left\|J_{x}-J_{x}^{\text {uniform }}\right\|,\left\|J_{y}-J_{y}^{\text {uniform }}\right\|$ and $\left\|B_{z}-B_{z}^{\text {uniform }}\right\|$ with respect to radius_ratio show more complex structure. In Figure 3.5, $\left\|J_{x}-J_{x}^{\text {uniform }}\right\|$ increases with the increasing radius_ratio all the time in the insulating case since the current path becomes more distinctive than the current path in uniform conductivity. The same behavior is seen in Figure 3.7 and Figure 3.9. However, in Figure 3.9, a decrease in $\left\|B_{z}-B_{z}^{\text {uniform }}\right\|$ is observed as the radius_ratio reaches to 0.85 . This is because a significant part of the magnetic flux density is generated outside the conductor region by the currents flowing on the boundaries of the conductor region and $B_{z}$ outside the conductor region is masked because of the reason mentioned before. Therefore, $\left\|B_{z}-B_{z}^{\text {uniform }}\right\|$ decreases after radius_ratio reaches to 0.85 . For the conductive case in Figure 3.5, $\left\|J_{x}-J_{x}^{\text {uniform }}\right\|$ decreases after radius_ratio reaches to 0.65 . This is because of the fact that the radius of the inhomogeneity becomes comparable to the radius of the conductor region and the current density distribution in the inhomogeneity becomes similar to the current density distribution in uniform

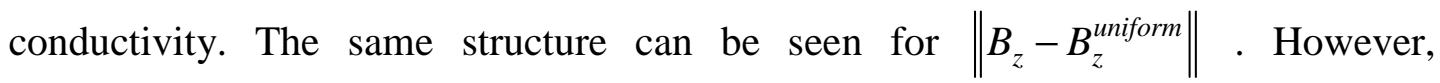
$\left\|J_{y}-J_{y}^{u n i f o r m}\right\|$ is always increasing as radius_ratio increases. This is because the currents flowing on the boundaries of the conductor region begin to flow into the inhomogeneity as the radius_ratio increases and this current flow orthogonal to the current injection pattern increases $\left\|J_{y}-J_{y}^{u n i f o r m}\right\|$.

$S B_{z} J_{x}$ and $S B_{z} J_{y}$ plots are given in Figure 3.10 and Figure 3.11. $S B_{z} J_{x}$ and $S B_{z} J_{y}$ are plotted with respect to the radius_ratio. $S B_{z} J_{x}$ and $S B_{z} J_{y}$ plots with respect to the sigma_ratio are linear as $\left\|J_{x}-J_{x}^{\text {uniform }}\right\|,\left\|J_{y}-J_{y}^{\text {uniform }}\right\|$ and $\left\|B_{z}-B_{z}^{\text {uniform }}\right\|$ show similar behaviours against the change in conductivity. 


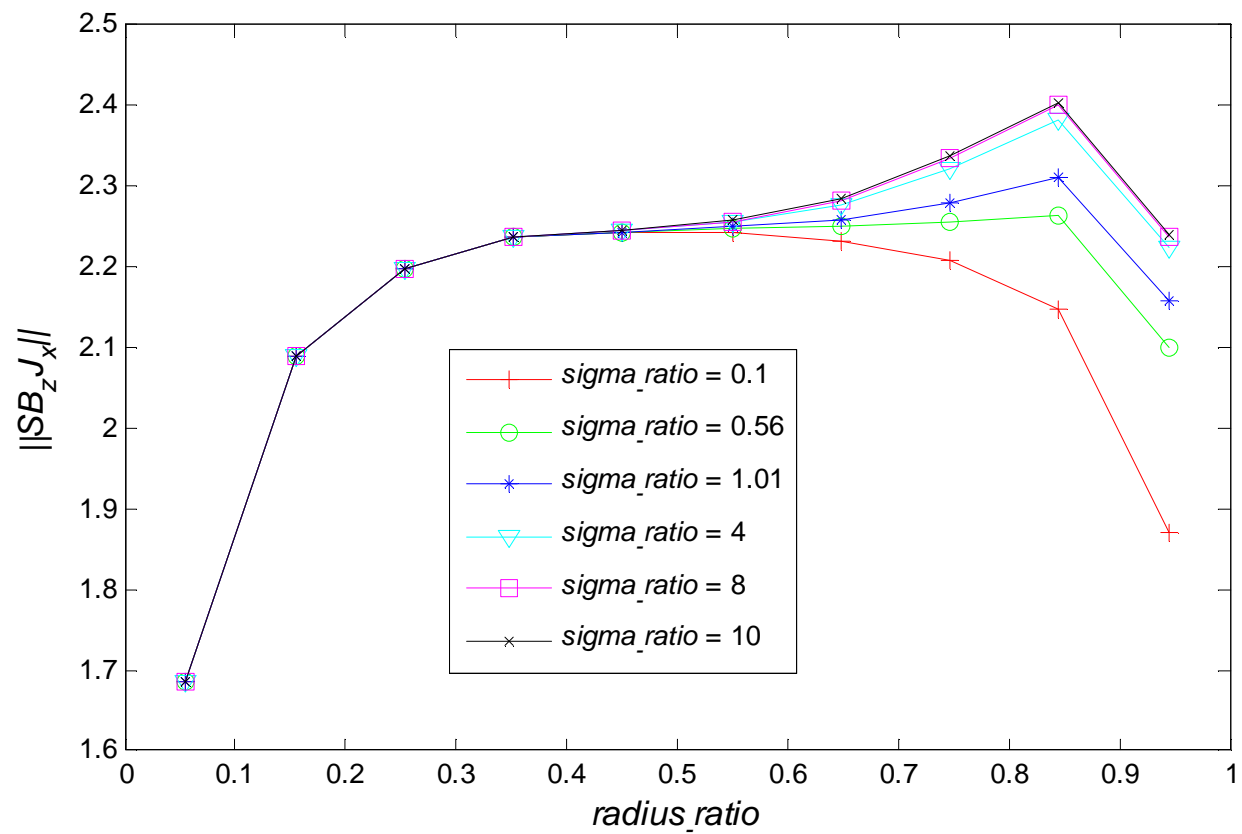

Figure $3.10 S B_{z} J_{x}$ vs. radius_ratio plot for various sigma_ratio values.

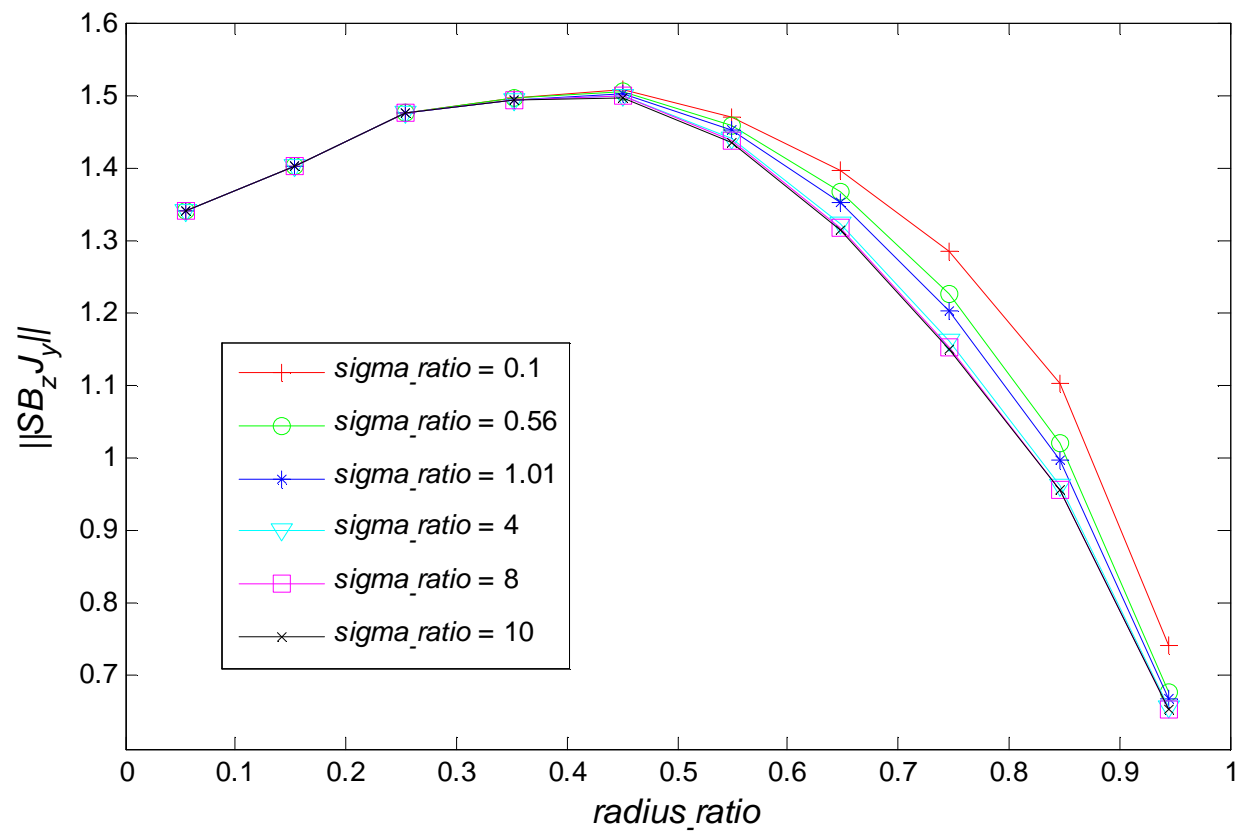

Figure $3.11 S B_{z} J_{y}$ vs. radius_ratio plot for various sigma_ratio values. 
In Figure 3.10, $S B_{z} J_{x}$ increases without any dependency on conductivity for the radius_ratio values up to 0.45 . The reason is that $B_{z}$ changes due to changes in $J_{x}$ and $J_{y}$. Therefore, the rate of change in $B_{z}$ is bigger until the radius_ratio reaches to 0.45 . As it was shown in Figure 3.5, $\left\|J_{x}-J_{x}^{\text {uniform }}\right\|$ begins to decrease with increasing radius_ratio after a point for conductive inhomogeneity and increases further for

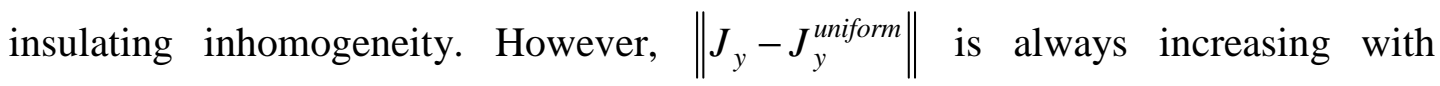
increasing radius_ratio. This effect can be clearly seen in the above figures. For $S B_{z} J_{x}$, the plots for various sigma_ratio begin to diverge from each other and increases for conductive inhomogeneity and decreases for insulating inhomogeneity. But, $S B_{z} J_{y}$ decreases for all sigma_ratio values. The sharp decrease at the end of both plots is possibly because of the masking of $B_{z}$.

\subsection{The Proposed Algorithm}

In this section, a novel current density reconstruction algorithm using only the $\mathrm{z}$ component of $\vec{B}, B_{z}$, is proposed. The Biot-Savart integral given in Section 3.2.1 is the main part of this algorithm. Hence, discretization of Biot-Savart integral, which will be carried out in Section 3.3.1, is the starting point.

\subsubsection{Discretization of Biot-Savart Integral}

In this section, a matrix relating current density and magnetic flux density is derived by using Biot-Savart law. Biot-Savart law in Equation (3.2) can be written as

$$
d \vec{B}=\frac{\mu_{0} I}{4 \pi}\left(\frac{d \vec{l}^{\prime} \times \vec{r}}{r^{3}}\right)
$$

which is the magnetic flux density due to a current element $I d \vec{l}^{\prime}$. Primed variables indicate the source points. Assume that for each element, current is localized at the center of corresponding element. Then, current element can be written in terms of current density and the area of corresponding element as

$$
I d \vec{l}^{\prime}=A\left(J_{x} \hat{a}_{x}+J_{y} \hat{a}_{y}+J_{z} \hat{a}_{z}\right)
$$


Also, the vector between the source and the field points, $\vec{r}$, can be written as

$$
\vec{r}=\left(x-x^{\prime}\right) \hat{a}_{x}+\left(y-y^{\prime}\right) \hat{a}_{y}+\left(z-z^{\prime}\right) \hat{a}_{z}
$$

The orthogonal components of magnetic flux density can be written as

$$
\begin{aligned}
& d B_{x}=\frac{\mu_{0} A}{4 \pi} \frac{\left(z-z^{\prime}\right) J_{y}-\left(y-y^{\prime}\right) J_{z}}{\left[\left(x-x^{\prime}\right)^{2}+\left(y-y^{\prime}\right)^{2}+\left(z-z^{\prime}\right)^{2}\right]^{\frac{3}{2}}} \\
& d B_{y}=\frac{\mu_{0} A}{4 \pi} \frac{\left(x-x^{\prime}\right) J_{z}-\left(z-z^{\prime}\right) J_{x}}{\left[\left(x-x^{\prime}\right)^{2}+\left(y-y^{\prime}\right)^{2}+\left(z-z^{\prime}\right)^{2}\right]^{\frac{3}{2}}} \\
& d B_{z}=\frac{\mu_{0} A}{4 \pi} \frac{\left(y-y^{\prime}\right) J_{x}-\left(x-x^{\prime}\right) J_{y}}{\left[\left(x-x^{\prime}\right)^{2}+\left(y-y^{\prime}\right)^{2}+\left(z-z^{\prime}\right)^{2}\right]^{\frac{3}{2}}}
\end{aligned}
$$

Evaluation of the above integrals gives the desired magnetic flux density distributions. Note that the effect of each current element on itself is neglected to overcome the singularity problem in evaluating the integrals. For our problem, we will deal with Equation (3.40) since current flowing in a plane (in our case x-y plane) creates a magnetic flux density only in the direction that is orthogonal to the plane (in our case z-direction). Now, assume that $2 \mathrm{D}$ conductor region, $\Omega$, is divided into $N$ finite element. Hence, $\mathbf{b}_{\mathbf{z}}$ can be written in matrix form as

$$
\left[\mathbf{b}_{\mathrm{z}}\right]=\left[\begin{array}{ll}
\mathbf{C}_{\mathbf{y}} & -\mathbf{C}_{\mathbf{x}}
\end{array}\right]\left[\begin{array}{l}
\mathbf{j}_{\mathbf{x}} \\
\mathbf{j}_{\mathbf{y}}
\end{array}\right]
$$

where $\mathbf{b}_{\mathbf{z}}, \mathbf{j}_{\mathbf{x}}$ and $\mathbf{j}_{\mathbf{y}}$ are $\mathrm{Nx} 1$ vectors and $\mathbf{C}_{\mathbf{x}}, \mathbf{C}_{\mathbf{y}}$ are $\mathrm{NxN}$ matrices. $\mathbf{C}_{\mathbf{x}}$ and $\mathbf{C}_{\mathbf{y}}$ matrices only depend on the vector between source and field points, $\vec{r}$. Hence, these matrices are constant for a given subject geometry.

\subsubsection{Difference Currents Concept}

If the current is applied to the $2 \mathrm{D}$ conductor region, $\Omega$, through the electrodes placed on each side, the current density distribution can be expressed as 


$$
\begin{aligned}
& \nabla \cdot \vec{J}=0 \text { in } \Omega \\
& \frac{\partial J}{\partial n}=g(x, y)
\end{aligned}
$$

where $g(x, y)$ is the applied current and it satisfies

$$
\begin{aligned}
& g(x, y)=0 \text { in } \Omega \\
& \int_{\partial \Omega} g(x, y) d s=0
\end{aligned}
$$

If $J(x, y)$ is assumed to be solenoidal, a differential equation relating $J_{x}$ and $J_{y}$ can be obtained. However, this assumption is not valid on boundaries since current is applied to the region. Difference currents concept is introduced to overcome this problem [25]. Difference currents are defined as

$$
\begin{aligned}
& J_{x}^{\text {diff }}=J_{x}-J_{x}^{\text {uniform }} \\
& J_{y}^{\text {diff }}=J_{y}-J_{y}^{\text {uniform }}
\end{aligned}
$$

Here, $J_{x}^{\text {uniform }}$ and $J_{y}^{\text {uniform }}$ are the currents for uniform conductivity distribution. In order to obtain these currents, a simulation model, which has the same geometry with the $2 \mathrm{D}$ conductor region, is prepared and its conductivity is chosen as uniform. Then, finite element method is used to obtain $J_{x}^{\text {uniform }}$ and $J_{y}{ }^{\text {uniform }}$. Note that the distribution of $\vec{J}$ is independent from the chosen uniform conductivity value. The divergences of total current density distribution and uniform current density distribution are the same. Hence, the difference current density distribution is solenoidal and a relation between $J_{x}^{\text {diff }}$ and $J_{y}^{\text {diff }}$ can be obtained as

$$
\frac{\partial J_{x}^{\text {diff }}}{\partial x}+\frac{\partial J_{y}^{\text {diff }}}{\partial y}=0
$$

The derivatives in Equation (3.45) can be approximated by using finite difference methods. In this study, central difference method is used as it yields a more accurate approximation than forward and backward difference methods. The discretization of Equation (3.45) shows that each element of $\mathbf{j}_{\mathbf{y}}$ diff vector can be expressed in terms of the elements of $\mathbf{j}_{\mathbf{x}}{ }^{\text {diff }}$ vector. Hence, an NxN matrix relating the elements of $\mathbf{j}_{\mathbf{x}}{ }^{\text {diff }}$ and $\mathbf{j}_{\mathbf{y}}{ }^{\text {diff }}$ vectors is formed as 


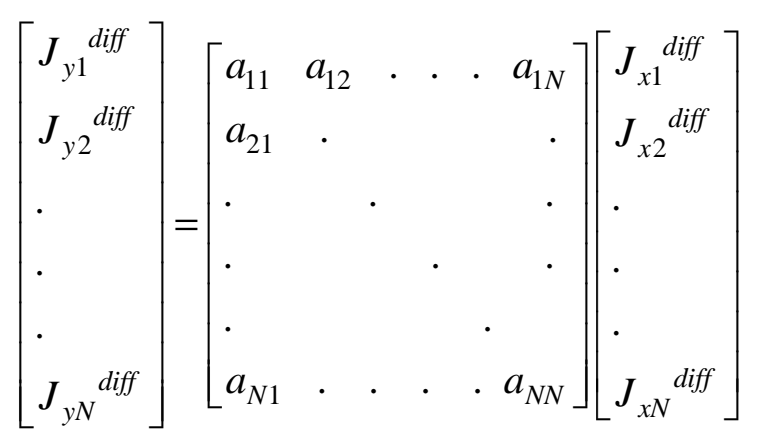

The elements of this A matrix is either zero or one depending on which elements of $\mathbf{j}_{\mathbf{x}}{ }^{\text {diff }}$ vector are related with the elements of $\mathbf{j}_{\mathbf{y}}{ }^{\text {diff }}$ vector. Also, the elements of $\mathbf{A}$ matrix depend on the subject geometry and number of finite elements, $N$. Equation (3.41) can be written for difference currents as

$$
\mathbf{b}_{\mathrm{z}}^{\text {diff }}=\mathbf{C}_{\mathbf{y}} \mathbf{j}_{\mathbf{x}}^{\text {diff }}-\mathbf{C}_{\mathbf{x}} \mathbf{j}_{\mathbf{y}}^{\text {diff }}
$$

Using Equation (3.46) and (3.47) together, the relation between $B_{z}^{\text {diff }}$ and $J_{x}^{\text {diff }}$ is obtained as

$$
\begin{gathered}
b_{z}^{\text {diff }}=\left(C_{y}-C_{x} A\right) j_{x}^{\text {diff }} \\
b_{z}^{\text {diff }}=C_{t} j_{x}^{\text {diff }}
\end{gathered}
$$

$\mathbf{b}_{\mathbf{z}}{ }^{\text {diff }}$ is the difference magnetic flux density vector which is calculated by subtracting $\mathbf{b}_{\mathbf{z}}{ }^{\text {uniform }}$ from the measured magnetic flux density vector, $\mathbf{b}_{\mathbf{z}} \cdot \mathbf{b}_{\mathbf{z}}{ }^{\text {uniform }}$ is generated by using uniform currents which are obtained from the simulation model. $\mathbf{j}_{\mathbf{x}}{ }^{\text {diff }}$ is calculated by using Equation (3.49) and then solution of $\mathbf{j}_{\mathbf{x}}{ }^{\text {diff }}$ is trivial. At last, $\mathbf{j}_{\mathbf{x}}$ and $\mathbf{j}_{\mathbf{y}}$ are obtained by adding difference currents and uniform currents. To summarize, the proposed algorithm has the following steps:

Step-1: Determine the subject geometry and number of finite elements, $N$,

Step-2: Calculate $\mathbf{C}_{\mathbf{x}}, \mathbf{C}_{\mathbf{y}}$ and $\mathbf{A}$ matrices,

Step-3: Calculate $\mathbf{C}_{\mathbf{t}}$,

Step-4: Compute $\mathbf{j}_{\mathbf{x}}{ }^{\text {diff }}$ using Equation (3.49), and then compute $\mathbf{j}_{\mathbf{y}}{ }^{\text {diff }}$.

The flowchart of the proposed algorithm is shown in Figure 3.12. 


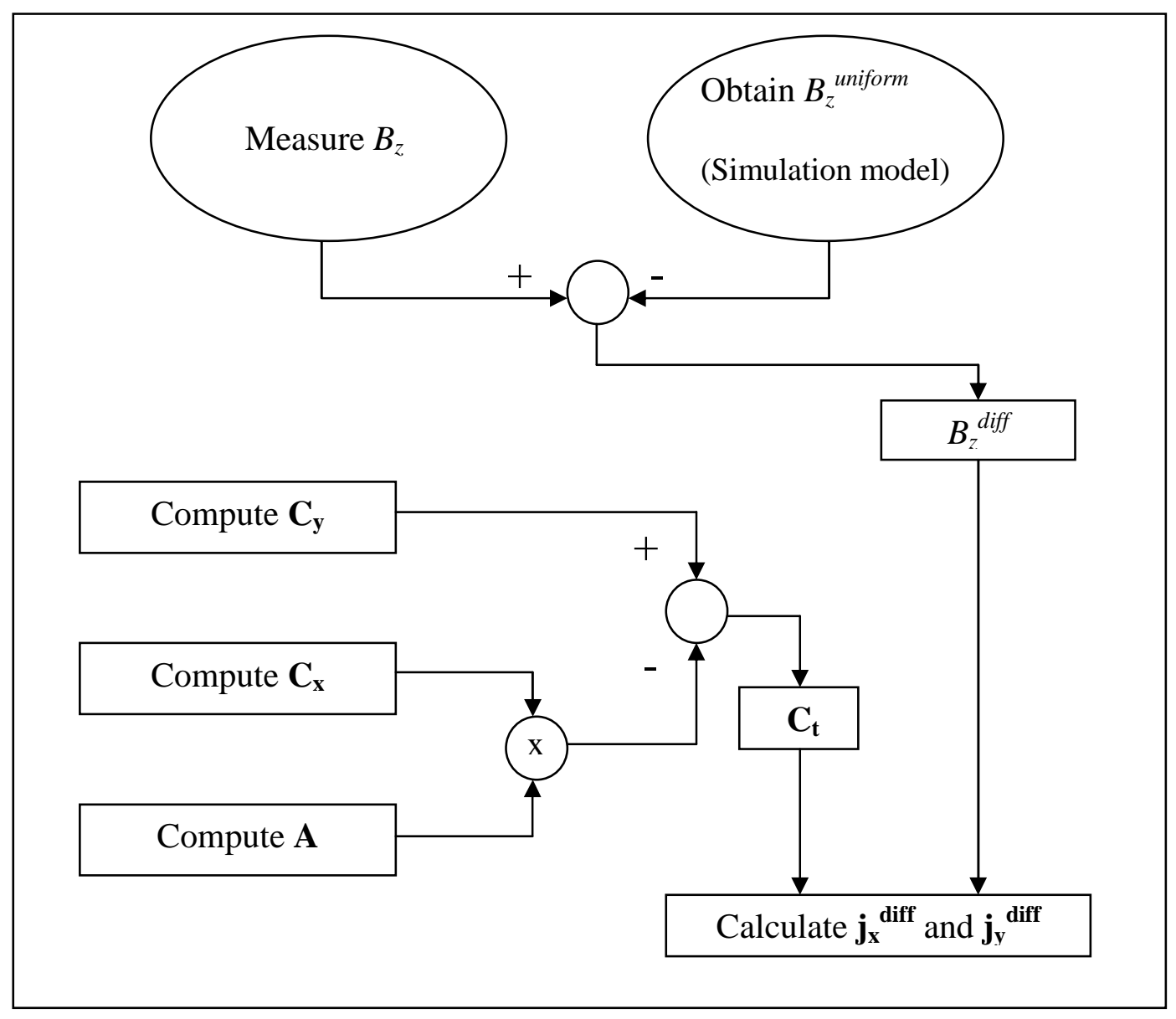

Figure 3.12 Flowchart of the proposed algorithm.

\subsection{Iterative FT-MRCDI Algorithm}

Ider (2006) proposed FT-MRCDI algorithm to reconstruct current density distribution using one component of magnetic flux density [25]. In this algorithm, Fourier Transform of $B_{z}$ is required to calculate Fourier Transforms of $J_{x}$ and $J_{y}$. Then $J_{x}$ and $J_{y}$ are calculated using inverse FT. In order to have a well-defined FT of $B_{z}, B_{z}$ must approach to zero at the boundaries of the conductor region. Since current flows at the boundaries of the conductor region, $B_{z}$ doesn't approach to zero at the boundaries. Hence, $B_{z}$ should be measured outside the conductor region to obtain a well-defined FT. It is known that measuring magnetic flux density outside the subject is not possible in MRI scanner. This is one of the major drawbacks of this algorithm. Ider et al proposed a novel algorithm named Iterative FT-MRCDI to overcome this problem [31]. In the iterative FT-MRCDI algorithm, $B_{z}$ outside the conductor region is found iteratively and $J_{x}$ and $J_{y}$ are calculated afterwards. 
The mathematical derivation of FT-MRCDI starts with the Biot-Savart integral. The Biot-Savart integral for the currents flowing in a $2 \mathrm{D}$ conductor region having a thickness, $d$, is given as

$$
B_{z}(x, y, z)=\frac{\mu_{0} d}{4 \pi} \int_{\Omega} \frac{J_{x}\left(x^{\prime}, y^{\prime}\right)\left(y-y^{\prime}\right)-\left(x-x^{\prime}\right) J_{y}\left(x^{\prime}, y^{\prime}\right)}{\left[\left(x-x^{\prime}\right)^{2}+\left(y-y^{\prime}\right)^{2}+z^{2}\right]^{\frac{3}{2}}} d x^{\prime} d y^{\prime}
$$

The Fourier Transform of $B_{z}(x, y, z)$ in $\mathrm{z}=0$ plane is given as

$$
\tilde{B}_{z}\left(k_{x}, k_{y}\right)=\frac{\mu_{0} d}{2}\left(\frac{j k_{y}}{k} \tilde{J}_{x}\left(k_{x}, k_{y}\right)-\frac{j k_{x}}{k} \tilde{J}_{y}\left(k_{x}, k_{y}\right)\right)
$$

where $\tilde{B}_{z}\left(k_{x}, k_{y}\right), \tilde{J}_{x}\left(k_{x}, k_{y}\right), \tilde{J}_{y}\left(k_{x}, k_{y}\right)$ are the 2D Fourier Transforms of $B_{z}(x, y)$, $J_{x}(x, y)$ and $J_{y}(x, y)$, respectively. Here, $k_{x}$ and $k_{y}$ are the components of spatial frequency, $\mathrm{k}$, which is defined as

$$
k=\sqrt{k_{x}^{2}+k_{y}^{2}}
$$

If the current is applied to the $2 \mathrm{D}$ conductor region, $\Omega$, through the electrodes placed on each side, the current density distribution can be written as

$$
\begin{aligned}
& \nabla \cdot \vec{J}=0 \text { in } \Omega \\
& \frac{\partial J}{\partial n}=g(x, y)
\end{aligned}
$$

where $g(x, y)$ is the applied current satisfying

$$
\begin{aligned}
& g(x, y)=0 \text { in } \Omega \\
& \int_{\partial \Omega} g(x, y) d s=0
\end{aligned}
$$

The Fourier Transform of Equation (3.53) is given as

$$
2 \pi j k_{x} \tilde{J}_{x}\left(k_{x}, k_{y}\right)+2 \pi j k_{y} \tilde{J}_{y}\left(k_{x}, k_{y}\right)=\tilde{g}\left(k_{x}, k_{y}\right)
$$

Solving Equations (3.51) and (3.55) together, one can obtain 


$$
\begin{gathered}
\tilde{J}_{x}\left(k_{x}, k_{y}\right)=-\frac{2 j k_{y}}{k \mu_{0} d} \tilde{B}_{z}\left(k_{x}, k_{y}\right)-\frac{j k_{x}}{2 \pi k^{2}} \tilde{g}\left(k_{x}, k_{y}\right) \\
\tilde{J}_{y}\left(k_{x}, k_{y}\right)=\frac{2 j k_{x}}{k \mu_{0} d} \tilde{B}_{z}\left(k_{x}, k_{y}\right)-\frac{j k_{y}}{2 \pi k^{2}} \tilde{g}\left(k_{x}, k_{y}\right)
\end{gathered}
$$

If the difference currents concept stated in Section 3.3.2 is applied to this problem, Equations (3.56) and (3.57) will become

$$
\begin{gathered}
\tilde{J}_{x}^{\text {diff }}\left(k_{x}, k_{y}\right)=-\frac{2 j k_{y}}{k \mu_{0} d} \tilde{B}_{z}^{\text {diff }}\left(k_{x}, k_{y}\right) \\
\tilde{J}_{y}^{\text {diff }}\left(k_{x}, k_{y}\right)=\frac{2 j k_{x}}{k \mu_{0} d} \tilde{B}_{z}^{\text {diff }}\left(k_{x}, k_{y}\right)
\end{gathered}
$$

Taking inverse FT of $\tilde{J}_{x}^{\text {diff }}\left(k_{x}, k_{y}\right)$ and $\tilde{J}_{y}^{\text {diff }}\left(k_{x}, k_{y}\right)$ gives the $J_{x}^{\text {diff }}$ and $J_{y}^{\text {diff }}$. Then, $J_{x}$ and $J_{y}$ are obtained by adding difference currents and uniform currents. As it was mentioned before, $B_{z}^{\text {diff }}$ should be measured outside the conductor region to obtain a well-defined FT. In the iterative FT-MRCDI algorithm, $B_{z}{ }^{\text {diff }}$ outside the conductor region is calculated iteratively. The iterative FT-MRCDI has the following steps:

Step-1: Let $\Omega_{m} \supset \Omega$ be a region where $B_{z}^{\text {diff }}$ can't be neglected,

Step-2: Take $B_{z}^{\text {diff }}$ as zero in $\Omega_{\mathrm{m}} \backslash \Omega$, and calculate $\tilde{B}_{z}^{\text {diff }}\left(k_{x}, k_{y}\right)$

Step-3: Calculate $\tilde{J}_{x}^{\text {diff }}\left(k_{x}, k_{y}\right)$ and $\tilde{J}_{y}^{\text {diff }}\left(k_{x}, k_{y}\right)$ using Equations (3.58) and (3.59),

Step-4: Find $J_{x}^{\text {diff }}$ and $J_{y}^{\text {diff }}$ by taking inverse FT, multiply with a supporting function that is one in $\Omega$ and zero in $\Omega_{\mathrm{m}} \backslash \Omega$ so that currents are restricted inside the conductor region,

Step-5: Calculate a new $B_{z}^{\text {diff }}$,

Step-6: Store $B_{z}^{\text {diff }}$ values in $\Omega_{\mathrm{m}} \backslash \Omega$ and change the values in $\Omega$ with measured ones,

Step-7: Return to Step-3 until the change in $B_{z}{ }^{\text {diff }}$ between successive iterations reduces below a predetermined error value. 
The flowchart of FT-MRCDI algorithm is shown in Figure 3.13.

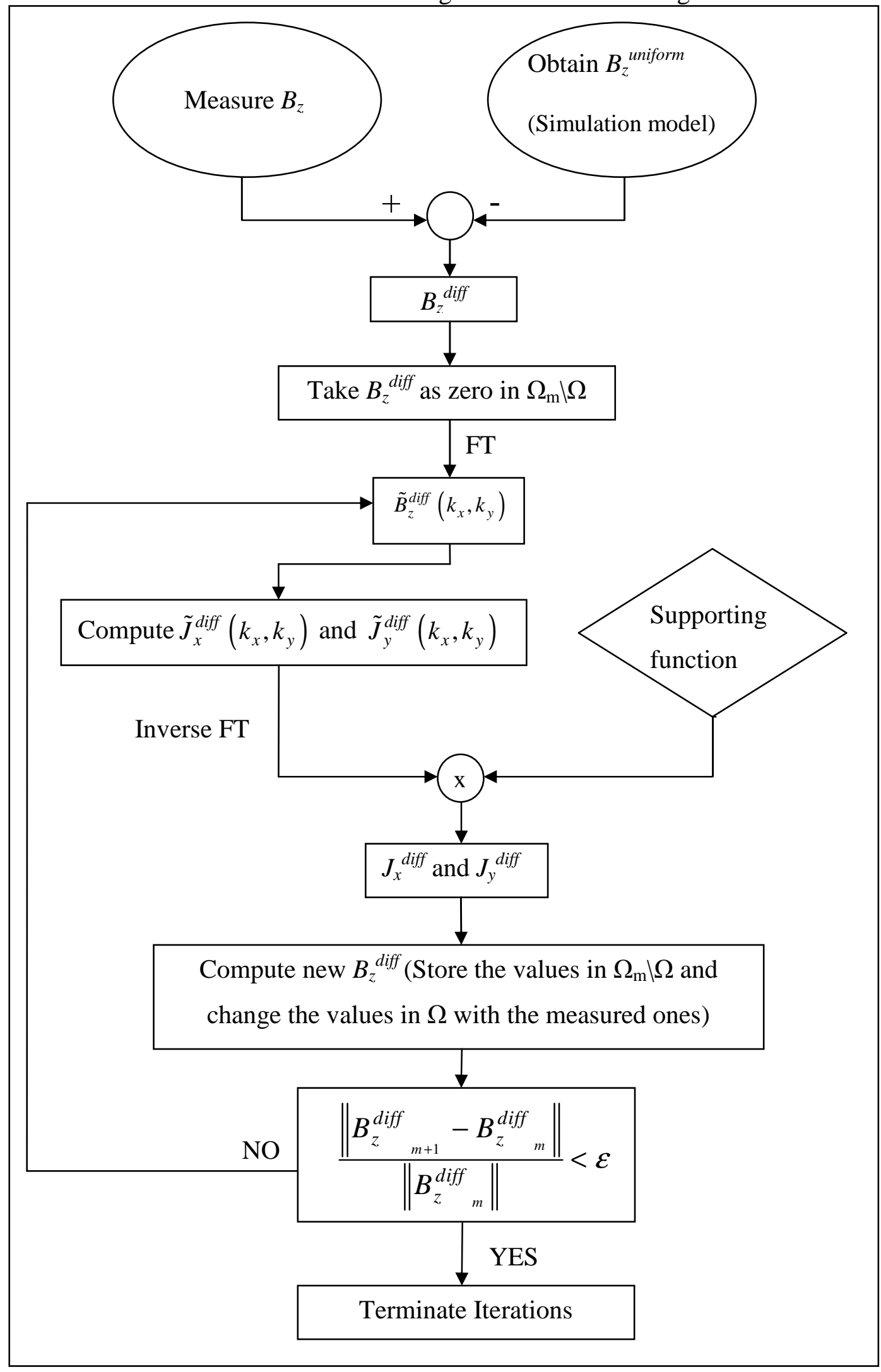

Figure 3.13 Flowchart of FT-MRCDI algorithm. 


\section{CHAPTER 4}

\section{SIMULATION AND EXPERIMENTAL MODELS}

\subsection{Introduction}

In this chapter, simulation and experimental models, which are prepared to evaluate the performance of the algorithms given in Chapter 3, will be explained. In the first part, three simulation and three experimental models will be presented. The procedure followed in preparing experimental phantom will be given afterwards. In the last part, error calculation in simulation and experimental models will be given.

\subsection{Models}

There are some important factors affecting the preparation of both simulation and experimental models. First of all, the experiments are performed in 0.15 T METUEE MRI System and the bore of this system is $80 \mathrm{~cm}$. Hence, sizes of RF coil and experimental phantom are restricted by the diameter of the bore. By taking this limitation into account, a new RF coil and experimental phantom were designed as big as possible. As a result, the experimental phantom was constructed with size of $9 \times 9 \times 9 \mathrm{~cm}^{3}$ and simulation phantoms were chosen the same as experimental phantom for consistency.

The MRCDI algorithms given in the previous chapter are 2D algorithms. However, the constructed experimental phantom has a 3D geometry. The $2 \mathrm{D}$ geometry is obtained with additional Plexiglas ${ }^{\circledR}$ walls which force the applied current to flow in a volume of 9x9x2 $\mathrm{cm}^{3}$. Electrodes are placed in the middle of each side and their dimensions are $2 \times 2 \mathrm{~cm}^{2}$ for both experimental and simulation phantoms except 
simulation model 1. In simulation model 1, the electrode size is chosen as the length of the phantom. For most cases, two current injection patterns are used. The first current injection pattern is vertical current injection pattern where current is injected from upside electrode and sunk from downside electrode. The other one is horizontal current injection pattern where current is injected from the electrode on the left and sunk from the electrode on the right. $20 \mathrm{~mA}$ current is injected to the phantom in all experiments.

Another important factor is the pixel size. The resolution of METU-EE MRI System limits the pixel size. Since the resolution of the system is $2 \mathrm{~mm}$, experimental phantoms are discretized into $44 \times 44$ pixels. In order to minimize the contribution of numerical error to the overall reconstruction error of the algorithms, simulation phantoms are discretized into 100x100 pixels. Hence, reconstruction performances of the algorithms can be evaluated more accurately.

\subsubsection{Simulation Models}

\subsubsection{Simulation Model 1}

The first simulation model is shown in Figure 4.1.

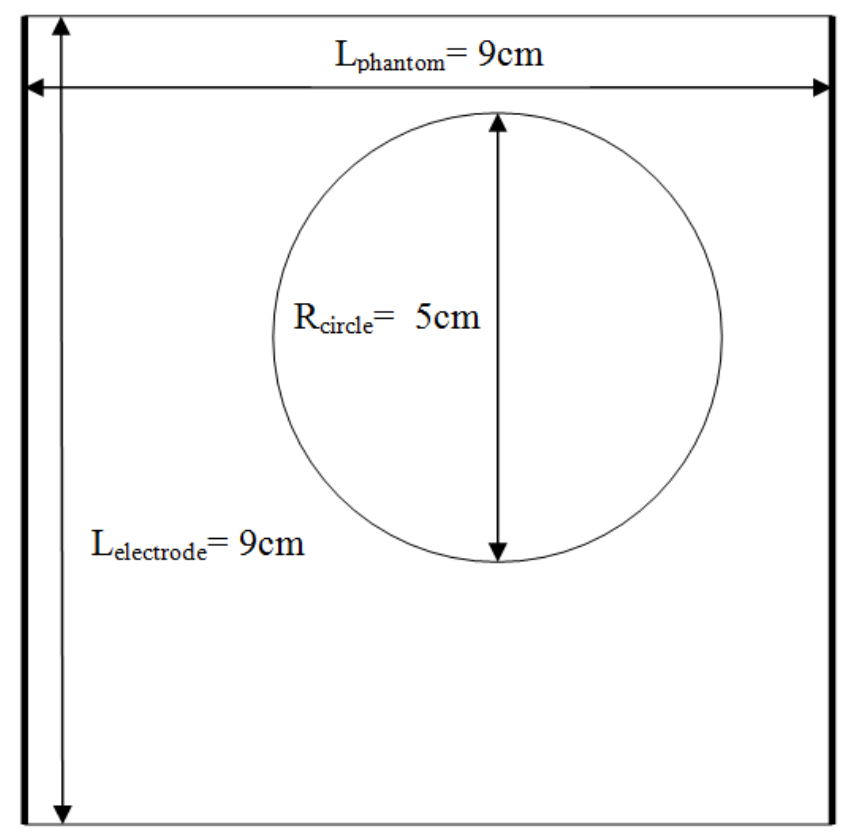

Figure 4.1 The geometry of Simulation Model 1. 
This model has a simple geometry with one circular object. The circular object has a conductivity of $1 \mathrm{~S} / \mathrm{m}$. It is placed on a background with a conductivity of $0.2 \mathrm{~S} / \mathrm{m}$. In this model, the conductivity transition between the background and the object is smoothened. The distinctive property of this simulation model is that large electrodes are used in this phantom. Therefore, the effect of electrode size in reconstruction performance can be investigated.

\subsubsection{Simulation Model 2}

The second simulation model is shown in Figure 4.2. This model contains two objects: a circular object having a conductivity of $2 \mathrm{~S} / \mathrm{m}$ and a square object prepared as a pure insulator. They are placed on a background with a conductivity of $0.2 \mathrm{~S} / \mathrm{m}$. The conductivity transition between the background and the object is also smoothened. This model has a more complex geometry than the first simulation model. It is designed to see the current paths for the cases where both conductive and insulating objects exist.

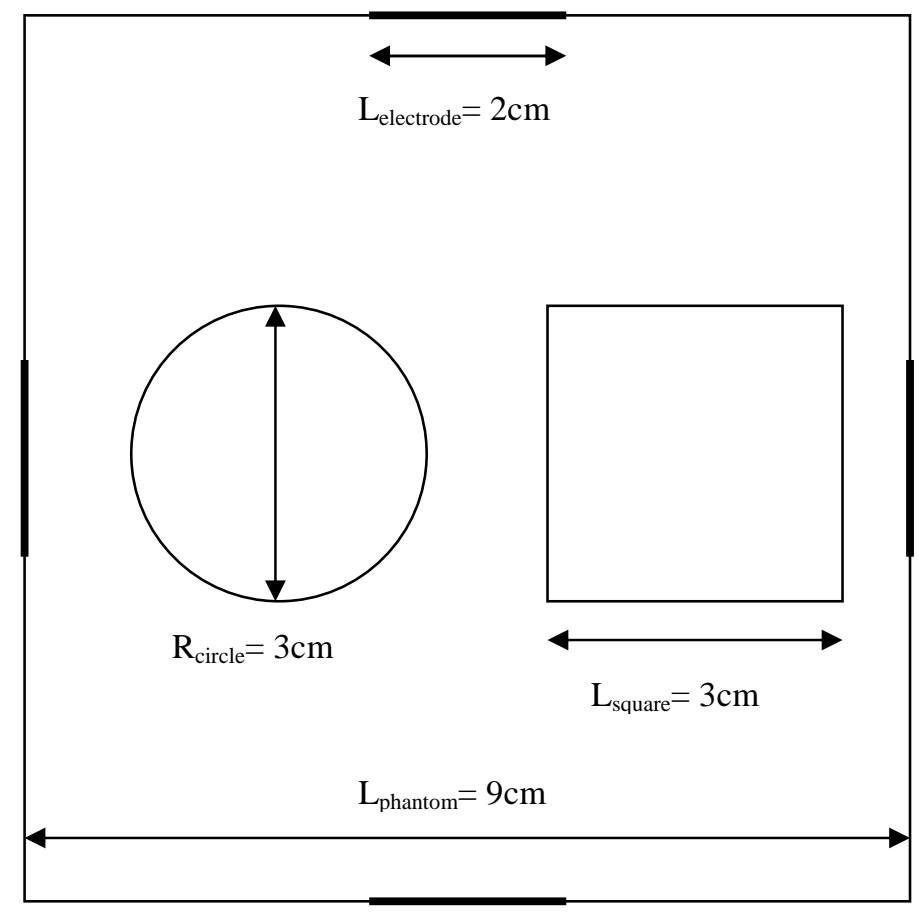

Figure 4.2 The geometry of Simulation Model 2. 


\subsubsection{Simulation Model 3}

This model has a square object placed at the center of the phantom as seen in Figure 4.3. The square object has a conductivity of $2 \mathrm{~S} / \mathrm{m}$ and the background has a conductivity of $0.2 \mathrm{~S} / \mathrm{m}$. The insulating walls are placed at the top and bottom side of the square object so that in vertical current injection pattern, the current density will be distributed as if the object is a pure insulator. For horizontal current injection pattern, the insulating slices won't affect the current density distribution significantly. Note that the conductivity transition between the background and the object cannot be smoothened in this model because of the insulating walls.

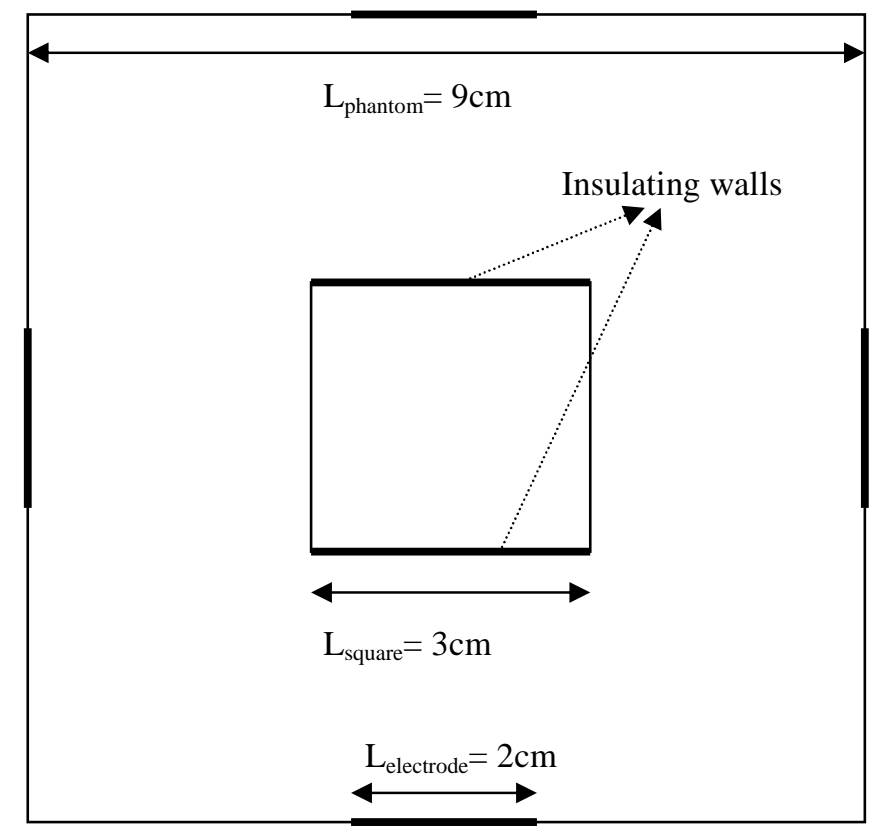

Figure 4.3 The geometry of Simulation Model 3.

\subsubsection{Experimental Models}

All the experimental models are prepared by using the experimental phantom. The experimental phantom has important features. First of all, recessed electrodes are used to eliminate the effect of high current density near the electrodes. Also, a table mechanism is designed in addition to the phantom to image exactly the same slice 
when the phantom is rotated. Two oblique CAD views of the phantom and the table mechanism are shown in Figure 4.4.
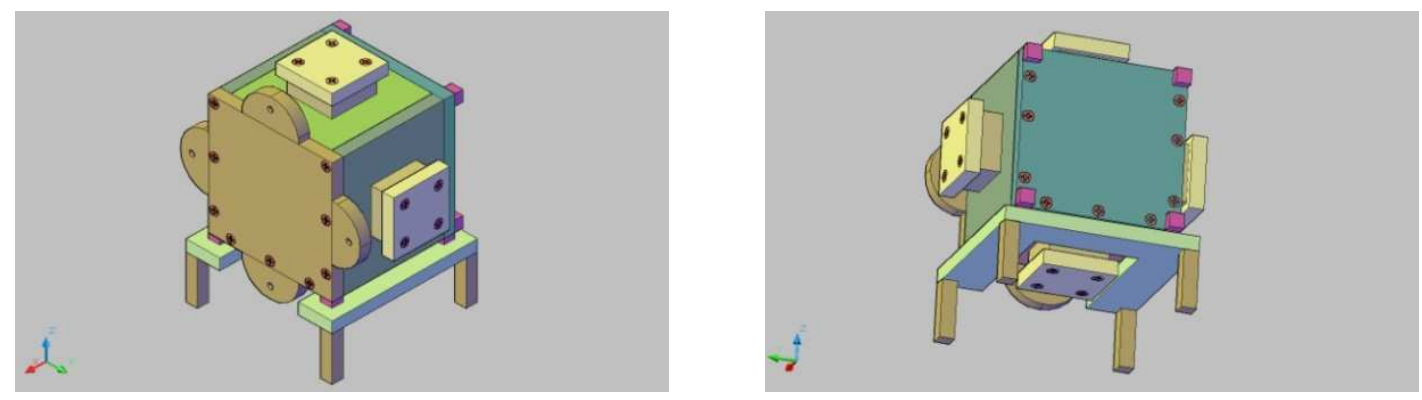

Figure 4.4 Two oblique views of CAD simulations of experimental phantom.

The constructed experimental phantom is shown in Figure 4.5.

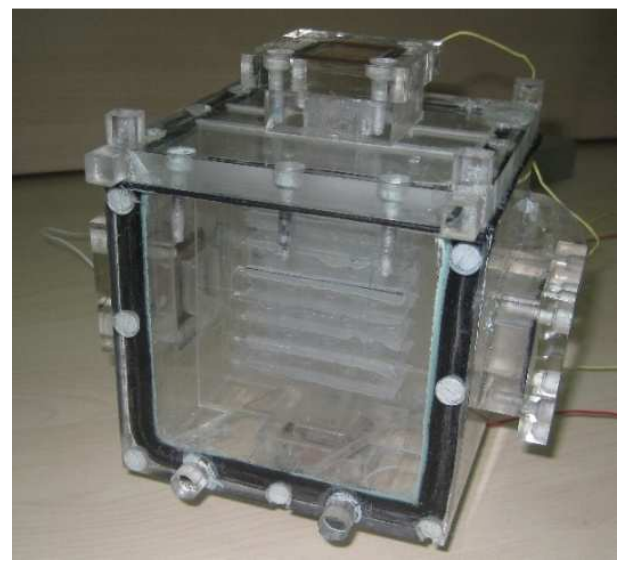

Figure 4.5 The experimental phantom.

In experimental models, the phantom elements and background are prepared by using solidifying materials, Agar-Agar, TX-150, TX-151. Also, NaCl is used to adjust conductivity values of elements and $\mathrm{CuSO} 4$ is used to fix $\mathrm{T} 1$ relaxation time.

\subsubsection{Experimental Model 1}

Experimental model 1 is the physical realization of the simulation model 2. As it was stated before, the model contains two objects: a circular object having a conductivity of $2 \mathrm{~S} / \mathrm{m}$ and a square object prepared as a pure insulator and they are placed on a 
background with a conductivity of $0.2 \mathrm{~S} / \mathrm{m}$. Composition of materials used to prepare the phantom elements are given in Table 4.1.

Table 4.1 Composition of phantom elements.

\begin{tabular}{|c|c|c|c|}
\hline & $\begin{array}{c}\text { Square object } \\
(\sim \mathbf{0} \mathbf{S} / \mathbf{m})\end{array}$ & $\begin{array}{c}\text { Circular object } \\
(\mathbf{2 ~ S} / \mathbf{m})\end{array}$ & $\begin{array}{c}\text { Background } \\
\mathbf{( 0 . 2} \mathbf{~ S} / \mathbf{m})\end{array}$ \\
\hline Distilled Water & $100 \mathrm{ml}$ & $100 \mathrm{ml}$ & $100 \mathrm{ml}$ \\
\hline Agar-Agar & $1 \mathrm{~g}$ & $1 \mathrm{~g}$ & - \\
\hline $\mathrm{TX}-150$ & - & - & $0.2 \mathrm{~g}$ \\
\hline $\mathrm{TX}-151$ & $1 \mathrm{~g}$ & $1 \mathrm{~g}$ & $2 \mathrm{~g}$ \\
\hline $\mathrm{NaCl}$ & - & $1.75 \mathrm{~g}$ & - \\
\hline $\mathrm{CuSO} 4$ & $0.1 \mathrm{~g}$ & $0.1 \mathrm{~g}$ & $0.1 \mathrm{~g}$ \\
\hline
\end{tabular}

Preparation procedure for circular object is as follows:

- Add $\mathrm{NaCl}$ to the distilled water and stir the solution,

- Add TX-151 and Agar-Agar and heat up the solution until it boils,

- Pour the solution to the circle mold.

The preparation procedure for square object is the same as the circle object but no $\mathrm{NaCl}$ is added to the solution. Also, stretch film is wrapped around the square object to adjust the conductivity of square element to $\sim 0 \mathrm{~S} / \mathrm{m}$. The preparation procedure for background is as follows:

- Add TX-150 and TX-151 to the distilled water,

- Stir the solution for ten minutes,

- Fill up the phantom with the prepared solution after placing the objects.

The phantom with all elements and MR magnitude image of the phantom are shown in Figure 4.6. 


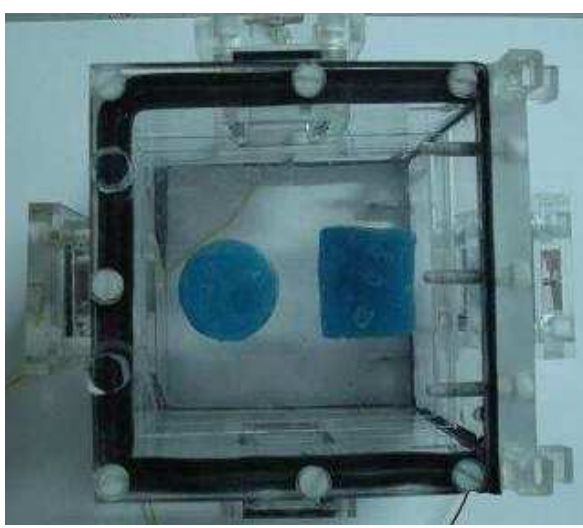

(a)

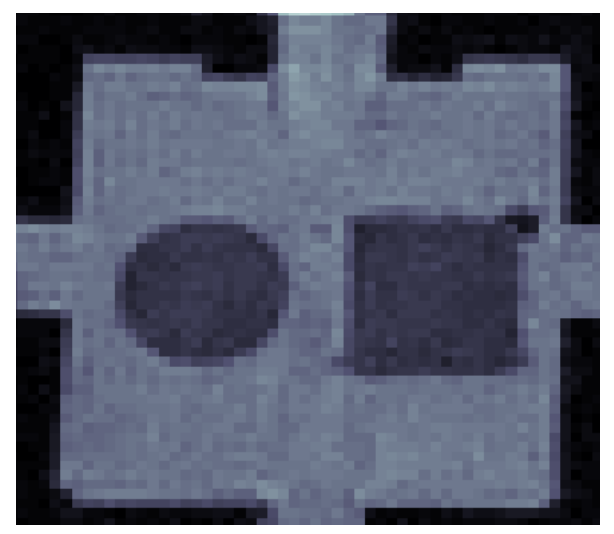

(b)

Figure 4.6 a) Top view of the phantom, b) MR magnitude image of the phantom.

\subsubsection{Experimental Model 2}

Experimental model 2 is the physical realization of the simulation model 3. As it was mentioned before, the model has a square object placed at the center of the phantom. The square object has a conductivity of $2 \mathrm{~S} / \mathrm{m}$ and the background has a conductivity of $0.2 \mathrm{~S} / \mathrm{m}$. The Plexiglas ${ }^{\circledR}$ walls are placed at the top and bottom side of the square object so that in vertical current injection pattern, the current density will be distributed as if the object is a pure insulator. The square object is prepared by using $100 \mathrm{ml}$ distilled water, $1 \mathrm{~g}$ Agar-Agar, $1 \mathrm{~g}$ TX-151, $1.75 \mathrm{~g} \mathrm{NaCl}, 0.1 \mathrm{~g} \mathrm{CuSO}_{4}$. The preparation procedure for circle object in experimental model 1 is followed while preparing the square object. The background in experimental model 1 is also used in this model. The phantom and its MR magnitude image are shown in Figure 4.7. 


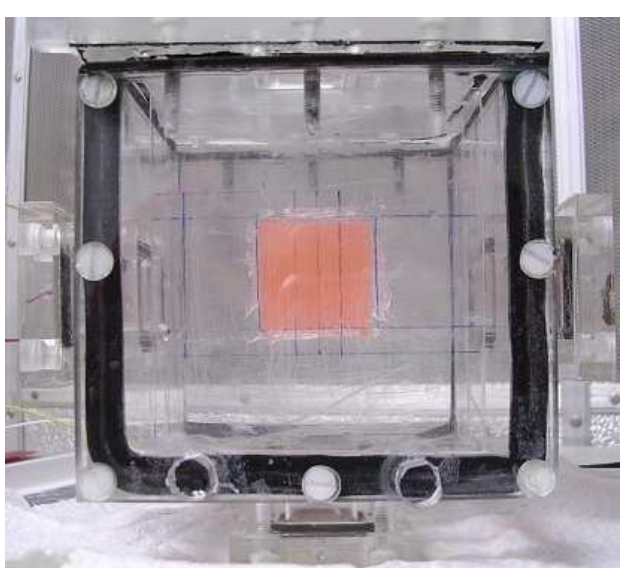

(a)

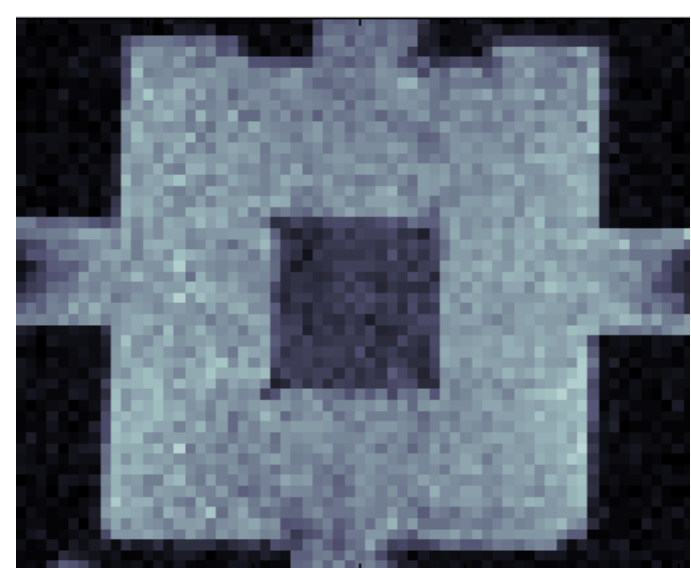

(b)

Figure 4.7 a) Experimental phantom, b) MR magnitude image of the phantom.

\subsubsection{Experimental Model 3}

In this model, 7 Plexiglas ${ }^{\circledR}$ slices are placed so that successive slices are separated by $8 \mathrm{~mm}$ distances. The dimensions of the Plexiglas ${ }^{\circledR}$ slices are $5.4 \times 2 \times 0.1 \mathrm{~cm}^{3}$. The first, third, fifth and seventh slices have 4 holes on them whereas the others have 3 holes. The diameter of the holes is $5 \mathrm{~mm}$. The phantom is filled with a solution having a conductivity of $2 \mathrm{~S} / \mathrm{m} .0 .1 \mathrm{~g} \mathrm{CuSO}_{4}$ and $0.145 \mathrm{~g} \mathrm{NaCl}$ are used while preparing the solution. The geometry of experimental model 3 is shown in Figure 4.8.

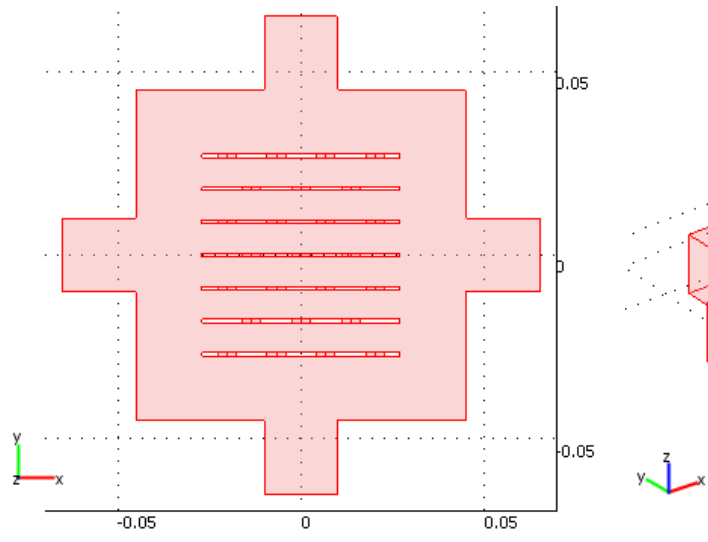

(a)

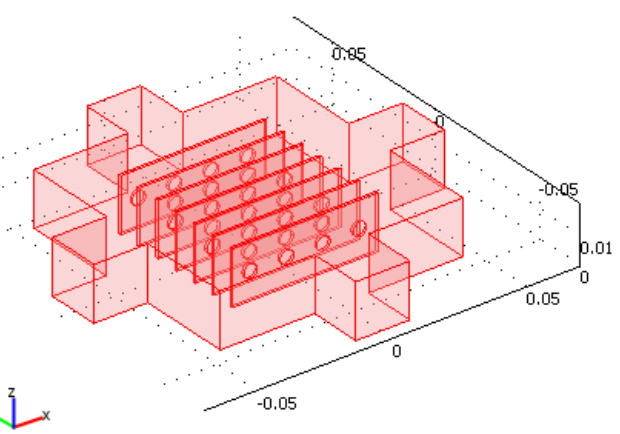

(b)

Figure 4.8 The geometry of Experimental Model 3, a) cross sectional view, b) 3D view. 
The aim of this model is to create an object that forces the current to flow between holes in vertical current injection pattern and image the different current paths occurred due to these holes. On the other hand, the insulating slices won't restrict the current density distribution significantly in horizontal current injection pattern. Hence, these insulating slices create local anistropic regions around the holes such that current flow in vertical direction is allowed whereas current flow in horizontal direction is not allowed. The phantom and its MR magnitude image are shown Figure 4.9.

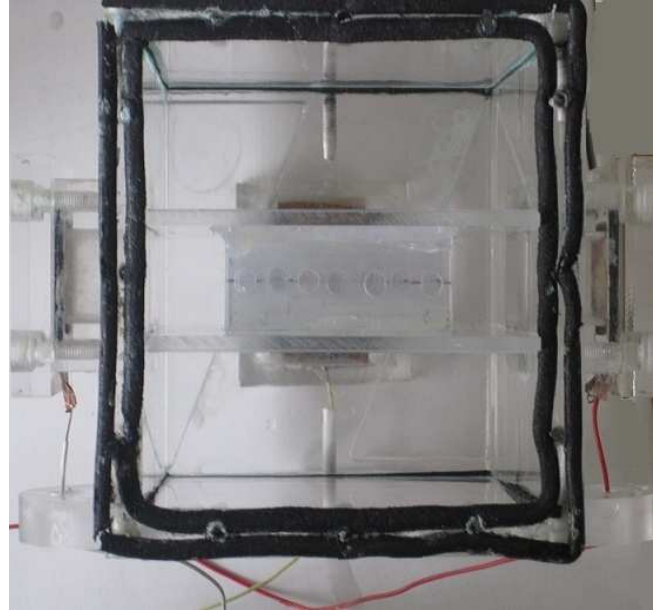

(a)

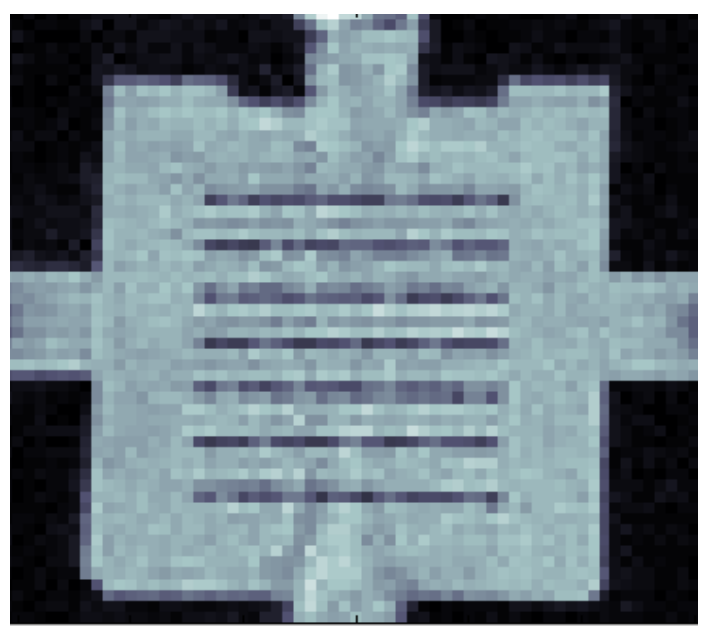

(b)

Figure 4.9 a) Top view of the phantom, b) MR magnitude image of the phantom.

\subsection{Noise Model for Computer Simulations}

A noise model is prepared in order to evaluate the performance of the algorithms on noisy data. In this study, random Gaussian noise model is used for noise simulations [32]. This noise model only depends on signal-to-noise ratio (SNR) of imaging system where magnetic flux density is measured. In this thesis, SNR 30 and SNR 13 levels are used in simulations since SNR 30 corresponds to a MRI system with 2T magnet and SNR value of 0.15 T METU-EE MRI system is around 13 [33].

The procedure of noisy data generation is as follows. First current density distribution is obtained by using Finite Element Method (FEM). Then, magnetic flux density is calculated by Biot-Savart law. Random Gaussian noise is added to 
magnetic flux density based on the SNR level of MRI System. Finally, noisy magnetic flux density is used in the MRCDI reconstruction algorithms. It is important to note that same Gaussian noise is added in all simulations for consistency.

\subsection{Error Calculation for Simulation Models}

Error calculation is carried out to evaluate the performances of the algorithms quantitatively. The error of reconstructed current density distribution is calculated using the following formula

$$
\varepsilon_{j}=\frac{\left\|J_{r}-J_{c}\right\|}{\left\|J_{r}\right\|}
$$

Here, $J_{r}$ and $J_{c}$ represent the real and calculated values of current density distribution respectively.

\subsection{Error Calculation for Experimental Models}

The error calculation for experimental models is not straightforward. It is known that the object to be imaged should be rotated inside the MRI scanner to measure all three components of $\vec{B}$. The phantom rotation causes artifacts in the reconstructed $\vec{J}$ due to variations in the internal geometry during rotation. Hence, $\vec{J}$ obtained by using three components of $\vec{B}$ deviates from the true current density. Therefore, quantitative evaluation of the reconstruction performance of the proposed algorithms on measured data, relative to the performance of the algorithms using all component of $\vec{B}$, is not possible. However, the reconstructed $\vec{J}$ can be verified by using divergence theorem and performance of the proposed algorithm can be evaluated in terms of error in the reconstructed MREIT conductivities, utilizing $J$-based MREIT algorithms on the reconstructed current density distributions.

\subsubsection{Verification Using Divergence Theorem}

From the divergence theorem, it is known that the integral of $\vec{J}$ in each slice which 
is selected orthogonal to the current injection pattern, should be the same and equal to the externally applied current. Hence, standard deviation of the integrals of $\vec{J}$ in the orthogonal slices should be zero. The standard deviations of the integrals of $\vec{J}$ for the algorithms give valuable information about the reconstruction performances of the algorithms.

\subsubsection{Evaluation in terms of Error in the Reconstructed MREIT Conductivities}

The performances of the MRCDI reconstruction algorithms are evaluated in terms of error in the reconstructed MREIT conductivities, utilizing J-substitution algorithm. Jsubstitution algorithm is chosen as it yields higher quality conductivity images compared to other reconstruction algorithms [18]. The J-substitution algorithm is proposed by Kwon et al [17]. The algorithm is an iterative algorithm which uses only the magnitude of current density inside the field of view. The algorithm tries to minimize a cost function which is defined as the difference between calculated and measured current density distributions.

Error for the reconstructed conductivity images are calculated as

$$
\varepsilon_{\sigma}=\sqrt{\frac{1}{N} \sum_{i=1}^{N} \frac{\left(\sigma_{i r}-\sigma_{i c}\right)^{2}}{\sigma_{i r}{ }^{2}}} \times 100
$$

where $N, \sigma_{i r}$ and $\sigma_{i c}$ are number of pixels, real and calculated conductivity values, respectively. 


\section{CHAPTER 5}

\section{RESULTS AND COMPARISON}

\subsection{Introduction}

In this chapter, reconstruction performances of the proposed algorithm and the iterative FT-MRCDI algorithm will be tested on both simulated and experimental data. In the first part, the reconstructed current density images will be presented with reconstruction errors for simulated data. In the second part, experimental results will be presented and conductivity images will be obtained from the reconstructed current density distributions, utilizing J-substitution algorithm. Then, reconstruction performance of the algorithms on experimental data will be evaluated in terms of error in the reconstructed conductivity images.

\subsection{Simulation Results}

\subsubsection{Results for Simulation Model 1}

The results obtained from simulation model 1 are presented in this subsection. The geometry and conductivity distribution of simulation model 1 was shown in Figure 4.1. As it was mentioned before, two noise levels, SNR 30 and SNR 13, were used in simulations. The procedure of generating noise levels and using them in simulations was discussed in Section 4.3.

In Figure 5.1, results, which are obtained by using the proposed algorithm and the iterative FT-MRCDI algorithm for noise-free case, are shown. Furthermore, reconstructed current density distributions for noise levels of SNR 30 and SNR 13 are shown in Figure 5.2 and Figure 5.3, respectively. 


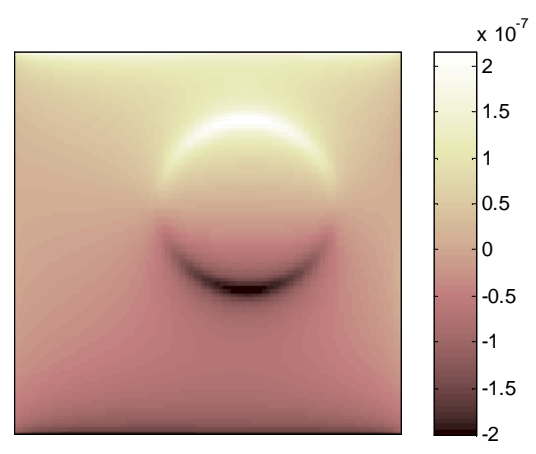

(a)

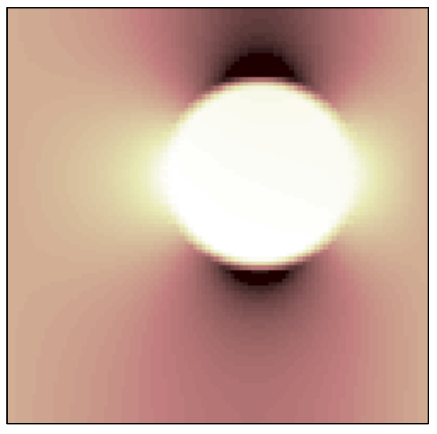

(b)

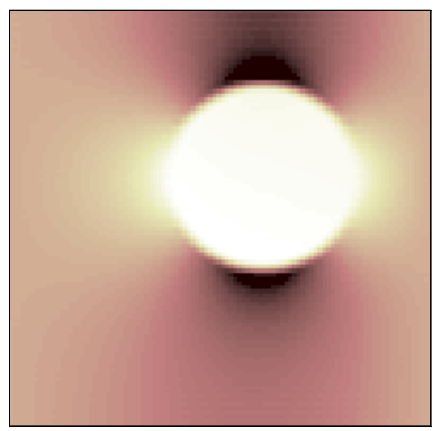

(d)

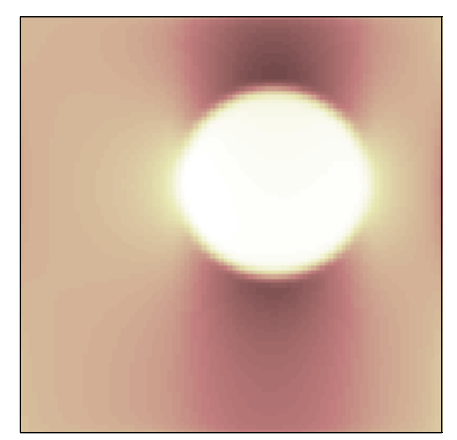

(f)
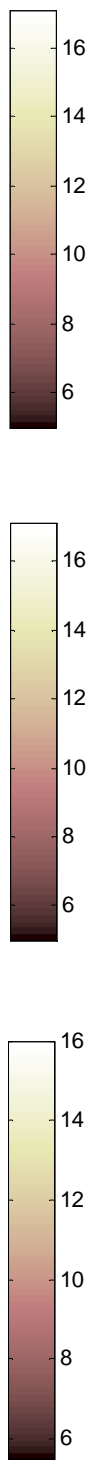

6

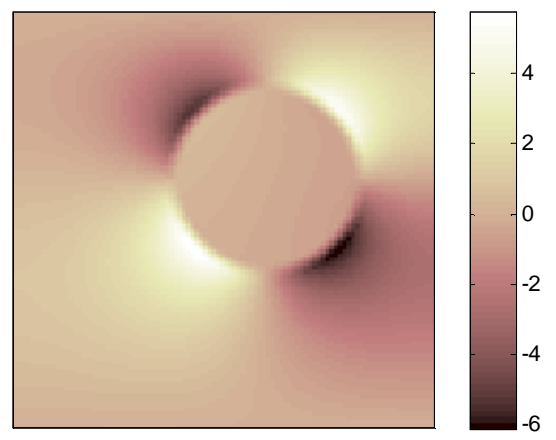

(c)

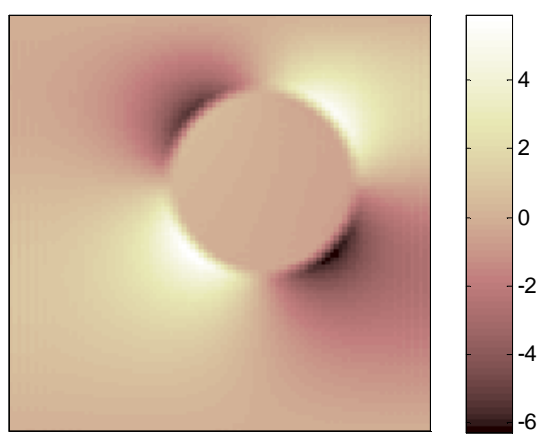

(e)

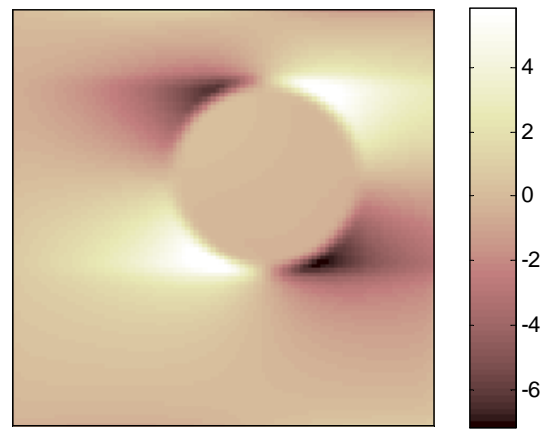

(g)

Figure 5.1 Results for noise-free case (horizontal $20 \mathrm{~mA}$ current injection) a) $B_{z}(\mathrm{~T})$, b) Original $J_{x}\left(\mathrm{~A} / \mathrm{m}^{2}\right)$ distribution, c) Original $J_{y}\left(\mathrm{~A} / \mathrm{m}^{2}\right)$ distribution, d) Reconstructed $J_{x}\left(\mathrm{~A} / \mathrm{m}^{2}\right)$ using the proposed algorithm, e) Reconstructed $J_{y}\left(\mathrm{~A} / \mathrm{m}^{2}\right)$ using the proposed algorithm, f) Reconstructed $J_{x}\left(\mathrm{~A} / \mathrm{m}^{2}\right)$ using Iterative FT-MRCDI algorithm, g) Reconstructed $J_{y}\left(\mathrm{~A} / \mathrm{m}^{2}\right)$ using Iterative FT-MRCDI algorithm. 


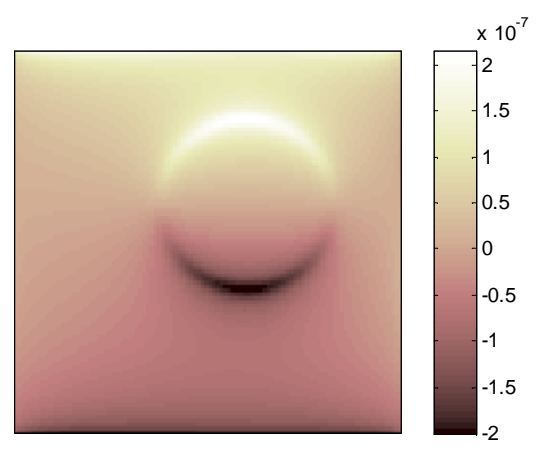

(a)

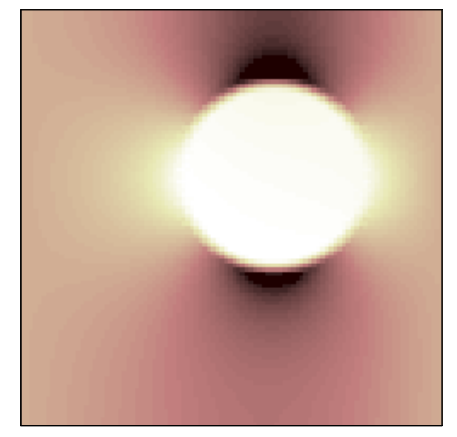

(b)

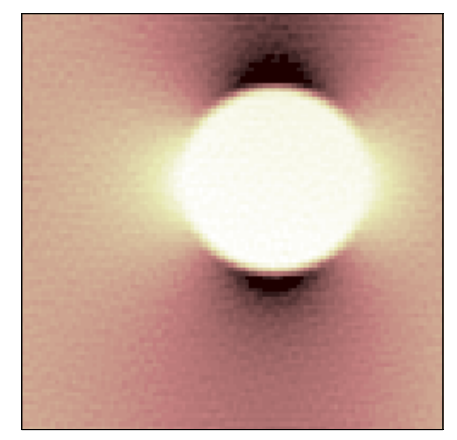

(d)

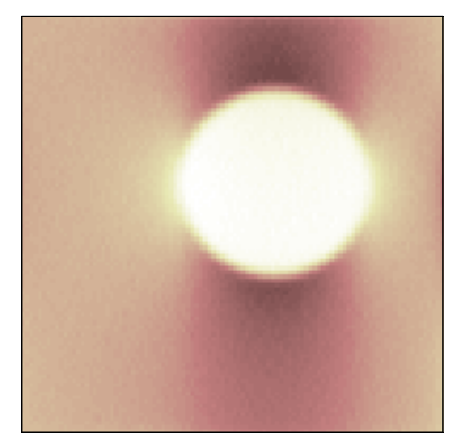

(f)
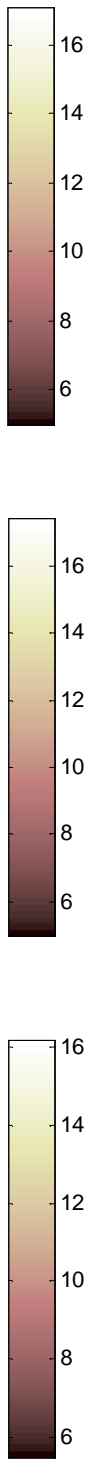
.

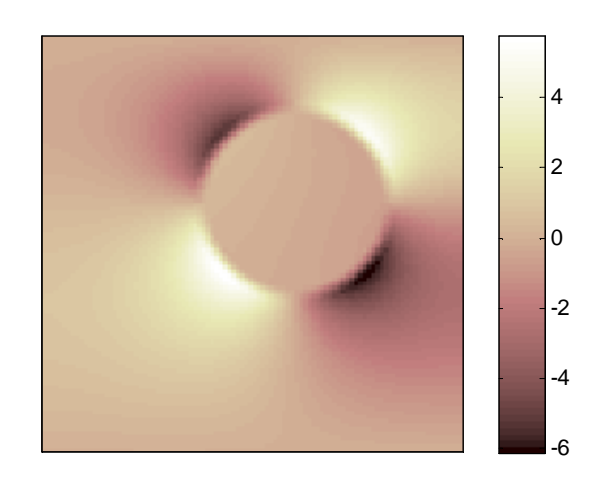

(c)

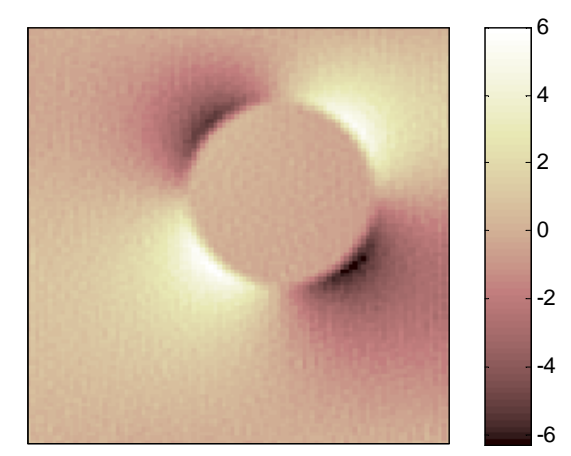

(e)

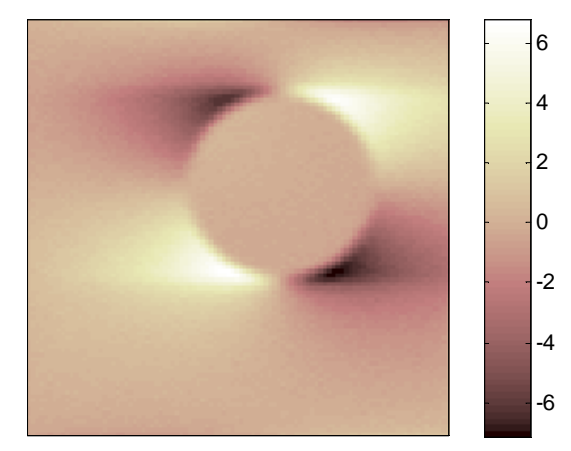

(g)

Figure 5.2 Results for SNR 30 case (horizontal $20 \mathrm{~mA}$ current injection) a) $B_{z}(\mathrm{~T})$, b) Original $J_{x}\left(\mathrm{~A} / \mathrm{m}^{2}\right)$ distribution, c) Original $J_{y}\left(\mathrm{~A} / \mathrm{m}^{2}\right)$ distribution, d) Reconstructed $J_{x}\left(\mathrm{~A} / \mathrm{m}^{2}\right)$ using the proposed algorithm, e) Reconstructed $J_{y}\left(\mathrm{~A} / \mathrm{m}^{2}\right)$ using the proposed algorithm, f) Reconstructed $J_{x}\left(\mathrm{~A} / \mathrm{m}^{2}\right)$ using Iterative FT-MRCDI algorithm, g) Reconstructed $J_{y}\left(\mathrm{~A} / \mathrm{m}^{2}\right)$ using Iterative FT-MRCDI algorithm. 


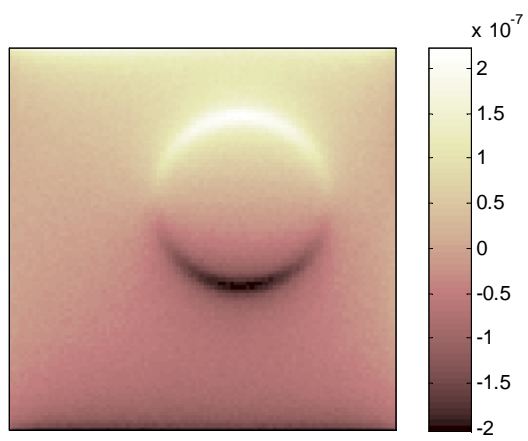

(a)

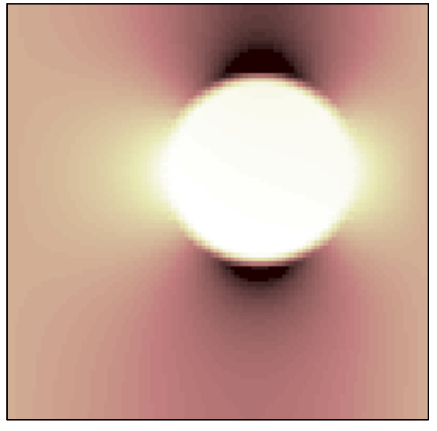

(b)

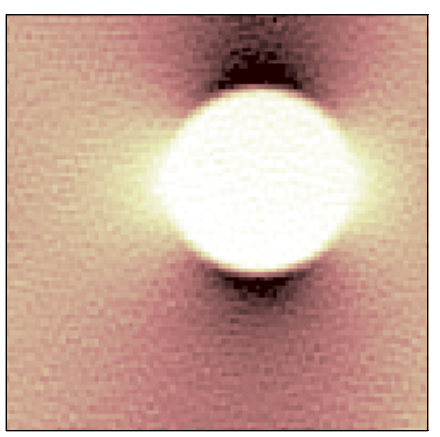

(d)

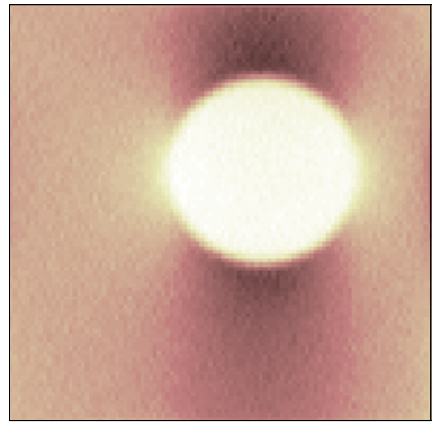

(f)
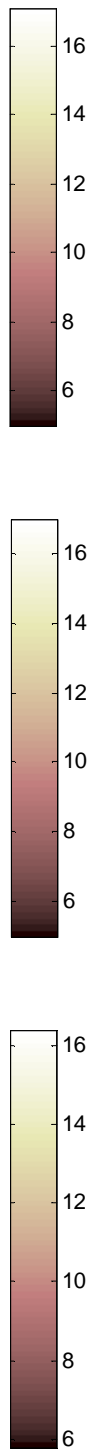

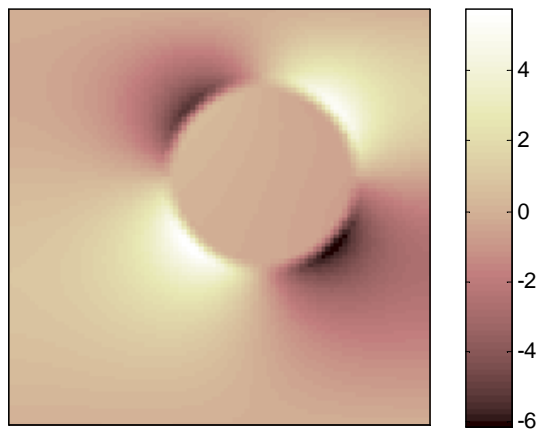

(c)

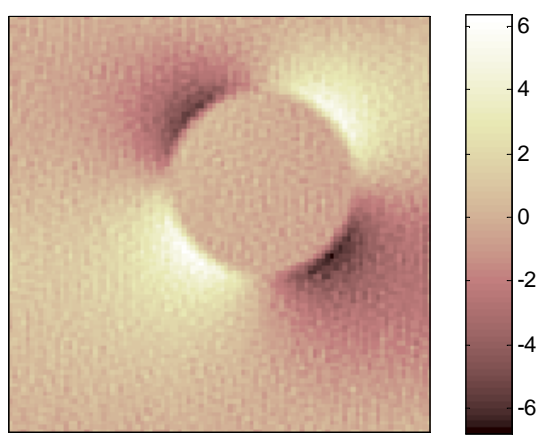

(e)

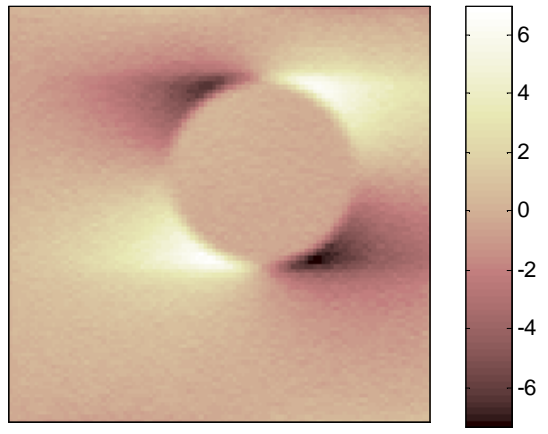

(g)

Figure 5.3 Results for SNR 13 case (horizontal $20 \mathrm{~mA}$ current injection) a) $B_{z}(\mathrm{~T})$, b) Original $J_{x}\left(\mathrm{~A} / \mathrm{m}^{2}\right)$ distribution, c) Original $J_{y}\left(\mathrm{~A} / \mathrm{m}^{2}\right)$ distribution, d) Reconstructed $J_{x}\left(\mathrm{~A} / \mathrm{m}^{2}\right)$ using the proposed algorithm, e) Reconstructed $J_{y}\left(\mathrm{~A} / \mathrm{m}^{2}\right)$ using the proposed algorithm, f) Reconstructed $J_{x}\left(\mathrm{~A} / \mathrm{m}^{2}\right)$ using Iterative FT-MRCDI algorithm, g) Reconstructed $J_{y}\left(\mathrm{~A} / \mathrm{m}^{2}\right)$ using Iterative FT-MRCDI algorithm. 
Although the reconstructed current density images give a perceptual idea on the reconstruction performance of both algorithms, quantitative evaluation of the reconstruction performances of the algorithms are listed in Table 5.1. The error calculation in reconstructed current density is carried out by using the error definition stated in Section 4.4 throughout this study.

When reconstruction performances of the algorithms are compared by evaluating the reconstructed current density images perceptually, it is seen that both algorithms have good reconstruction performances for noise-free case. For noisy cases, the effect of filtering is obvious in the iterative FT-MRCDI algorithm. Because of this filtering effect, it can be said that the performance of the iterative FT-MRCDI algorithm is not influenced much by the noise. In the proposed algorithm, the effect of noise is more apparent. However, quality of the reconstructed current density images is good.

Table 5.1 Errors in the reconstructed current density for simulation model 1.

\begin{tabular}{|c|c|c|c|c|c|c|}
\hline \multirow{2}{*}{} & \multicolumn{7}{|c|}{ Horizontal Current Injection } & \multicolumn{2}{c|}{ Vertical Current Injection } \\
\cline { 2 - 7 } & $J_{x}$ & $J_{y}$ & $J$ & $J_{x}$ & $J_{y}$ & $J$ \\
\hline \multicolumn{7}{|c|}{ The Proposed Algorithm } \\
\hline Noise-free & $0.27 \%$ & $2.44 \%$ & $0.30 \%$ & $2.44 \%$ & $0.27 \%$ & $0.30 \%$ \\
\hline SNR 30 & $1.35 \%$ & $9.57 \%$ & $1.35 \%$ & $10.14 \%$ & $1.38 \%$ & $1.36 \%$ \\
\hline SNR 13 & $3.09 \%$ & $21.47 \%$ & $3.06 \%$ & $23.00 \%$ & $3.33 \%$ & $3.27 \%$ \\
\hline \multicolumn{7}{|c|}{ Iterative FT-MRCDI Algorithm } \\
\hline Noise-free & $7.41 \%$ & $32.67 \%$ & $7.45 \%$ & $32.67 \%$ & $7.41 \%$ & $7.45 \%$ \\
\hline SNR 30 & $7.44 \%$ & $32.92 \%$ & $7.48 \%$ & $32.90 \%$ & $7.43 \%$ & $7.48 \%$ \\
\hline SNR 13 & $7.53 \%$ & $34.18 \%$ & $7.57 \%$ & $34.05 \%$ & $7.52 \%$ & $7.56 \%$ \\
\hline
\end{tabular}

Quantitative evaluation of the reconstruction performances of the algorithms shows that the proposed algorithm reconstructs with less error. It can be seen from Table 5.1 that the reconstruction performances of the algorithms for the component of current density orthogonal to the current injection pattern is poorer. This is expected since the component of current density in the direction of injected current is stronger. 
When the algorithms are compared according to the reconstruction time, the proposed algorithm has a shorter reconstruction time. This is an expected fact since the matrices used in the proposed algorithm are calculated once and stored for a subject with a specific geometry. However, the iterative FT-MRCDI algorithm calculates the difference magnetic flux density iteratively, which causes longer reconstruction time.

\subsubsection{Results for Simulation Model 2}

The results obtained from simulation model 2 are presented in this subsection. The geometry and conductivity distribution of simulation model 2 was shown in Figure 4.2. Two noise levels, SNR 30 and SNR 13, were also used in these simulations.

Z-component of magnetic flux density, $B_{z}$, obtained from horizontal and vertical current injection patterns are shown in Figure 5.4.

Reconstructed $J_{x}$ and $J_{y}$ for different noise levels for horizontal current injection pattern are shown in Figure 5.5 and Figure 5.6, respectively. For vertical current injection pattern, reconstructed $J_{x}$ and $J_{y}$ for different noise levels are shown in Figure 5.7 and Figure 5.8, respectively. Note that, the direction of positive y-axis is taken as negative in $J_{y}$ for vertical current injection to make the images consistent with $J_{x}$ images for horizontal current injection pattern.

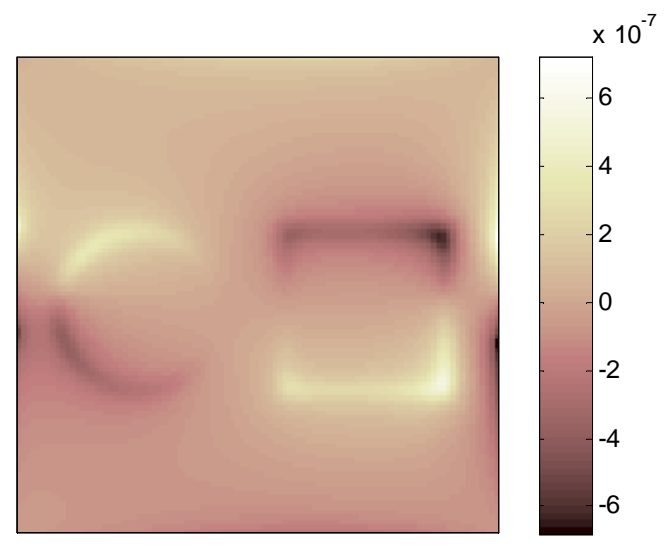

(a)

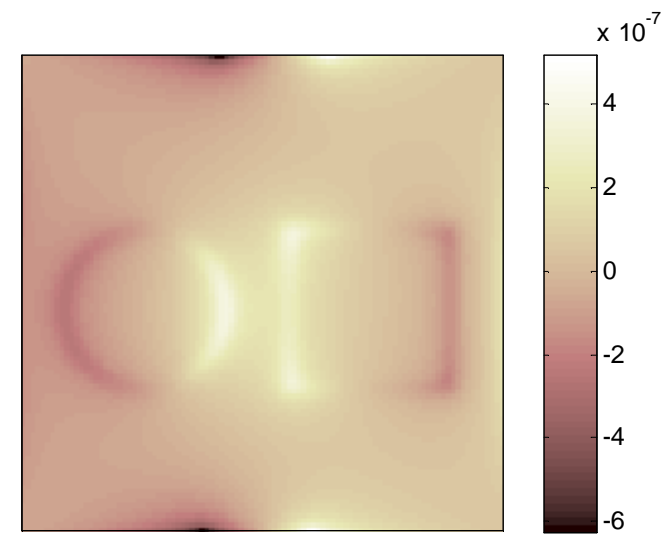

(b)

Figure 5.4 z-component of magnetic flux density, $B_{z}(\mathrm{~T})$ a) For horizontal current injection pattern, b) For vertical current injection pattern. 


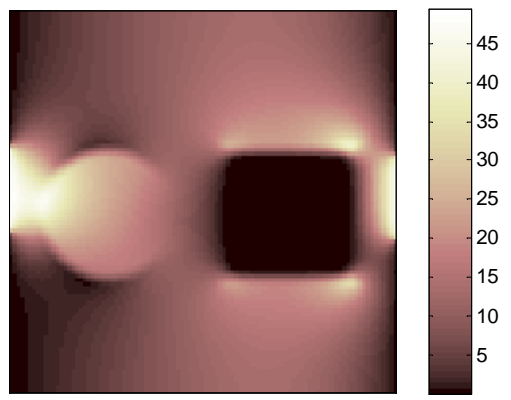

(a)

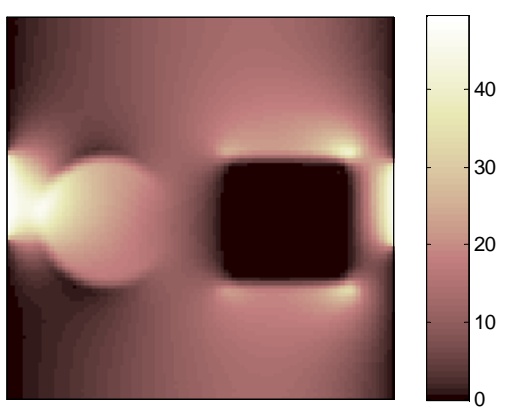

(b)

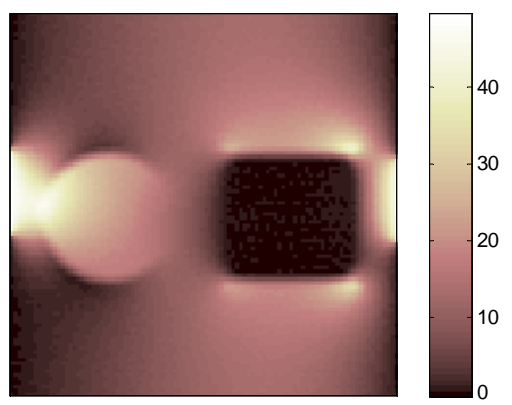

(d)

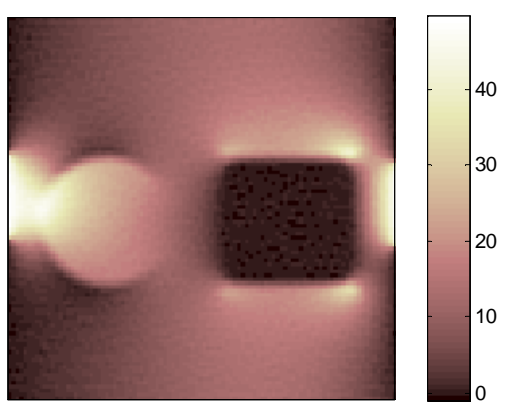

(f)

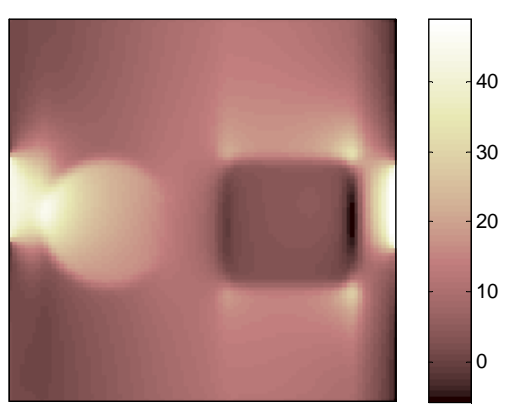

(c)

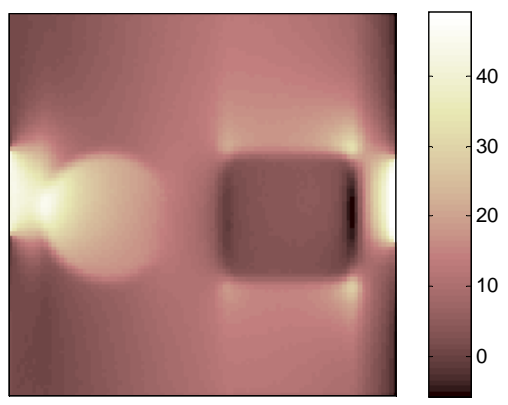

(e)

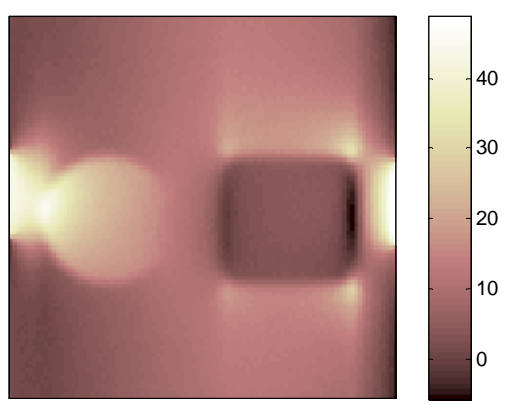

(g)

Figure 5.5 Reconstructed $J_{x}$ for different noise levels (horizontal $20 \mathrm{~mA}$ current injection) a) Original $J_{x}\left(\mathrm{~A} / \mathrm{m}^{2}\right)$ distribution, b) Reconstructed $J_{x}\left(\mathrm{~A} / \mathrm{m}^{2}\right)$ using the proposed algorithm for noise-free case, c) Reconstructed $J_{x}\left(\mathrm{~A} / \mathrm{m}^{2}\right)$ using Iterative FT-MRCDI algorithm for noise-free case, d) Reconstructed $J_{x}\left(\mathrm{~A} / \mathrm{m}^{2}\right)$ using the proposed algorithm for SNR30 case, e) Reconstructed $J_{x}\left(\mathrm{~A} / \mathrm{m}^{2}\right)$ using Iterative FTMRCDI algorithm for SNR 30 case, f) Reconstructed $J_{x}\left(\mathrm{~A} / \mathrm{m}^{2}\right)$ using the proposed algorithm for SNR13 case, g) Reconstructed $J_{x}\left(\mathrm{~A} / \mathrm{m}^{2}\right)$ using Iterative FT-MRCDI algorithm for SNR 13 case. 


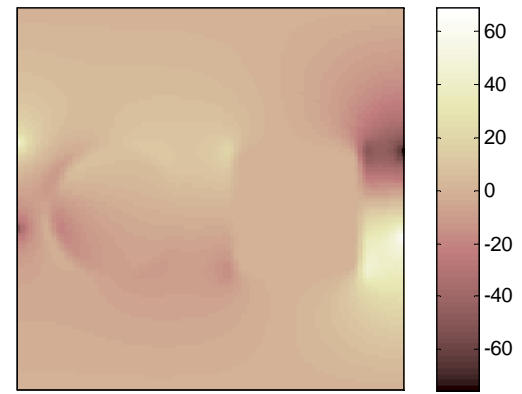

(a)

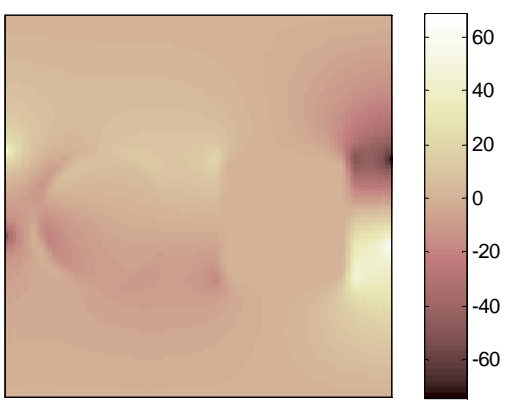

(b)

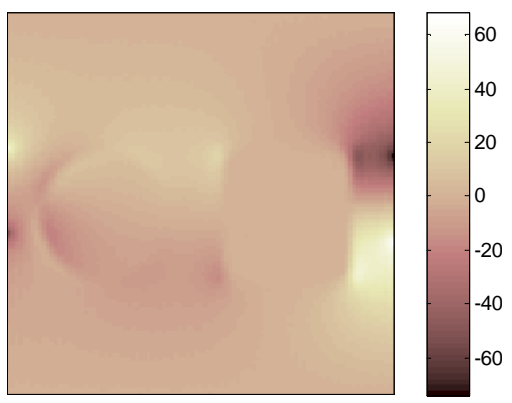

(d)

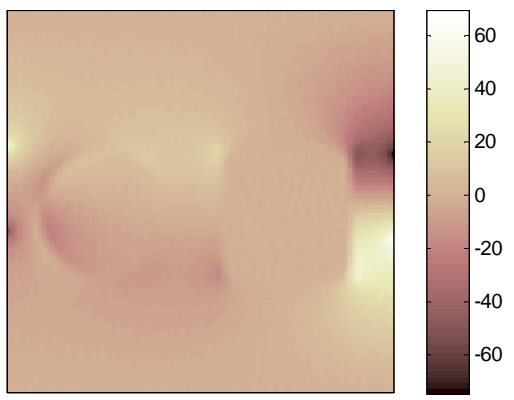

(f)

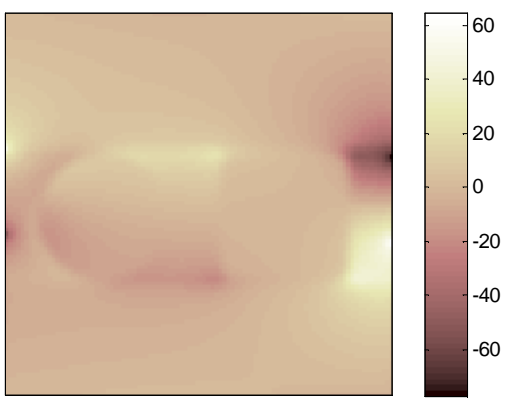

(c)

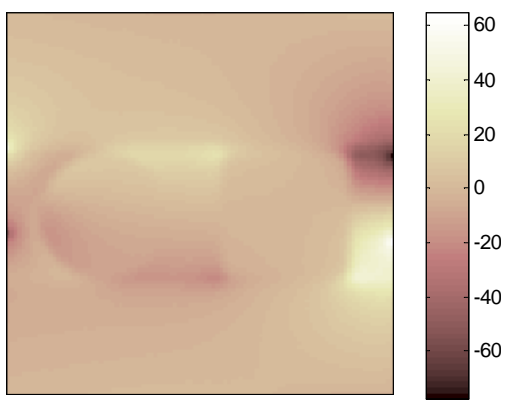

(e)

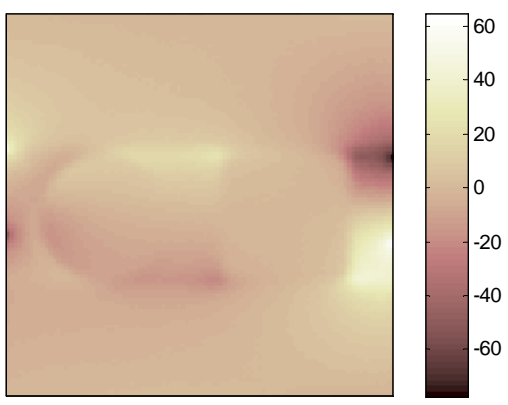

(g)

Figure 5.6 Reconstructed $J_{y}$ for different noise levels (horizontal $20 \mathrm{~mA}$ current injection) a) Original $J_{y}\left(\mathrm{~A} / \mathrm{m}^{2}\right)$ distribution, b) Reconstructed $J_{y}\left(\mathrm{~A} / \mathrm{m}^{2}\right)$ using the proposed algorithm for noise-free case, c) Reconstructed $J_{y}\left(\mathrm{~A} / \mathrm{m}^{2}\right)$ using Iterative FT-MRCDI algorithm for noise-free case, d) Reconstructed $J_{y}\left(\mathrm{~A} / \mathrm{m}^{2}\right)$ using the proposed algorithm for SNR30 case, e) Reconstructed $J_{y}\left(\mathrm{~A} / \mathrm{m}^{2}\right)$ using Iterative FTMRCDI algorithm for SNR 30 case, f) Reconstructed $J_{y}\left(\mathrm{~A} / \mathrm{m}^{2}\right)$ using the proposed algorithm for SNR13 case, g) Reconstructed $J_{y}\left(\mathrm{~A} / \mathrm{m}^{2}\right)$ using Iterative FT-MRCDI algorithm for SNR 13 case. 


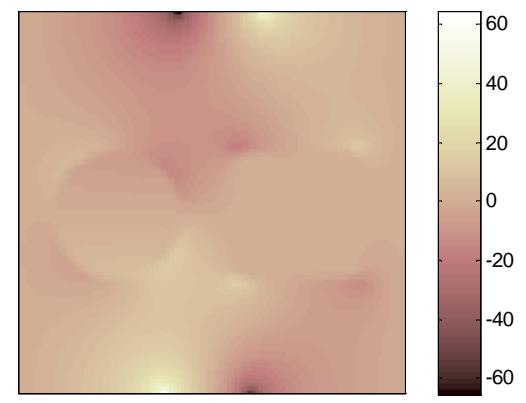

(a)

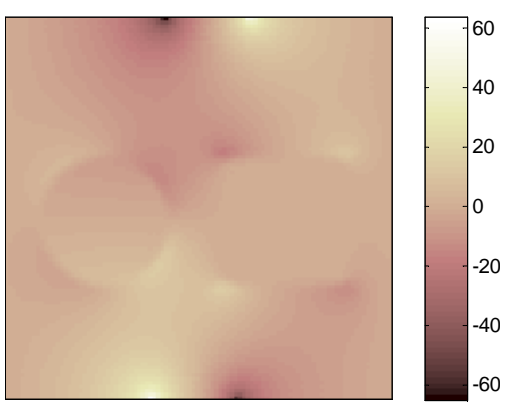

(b)

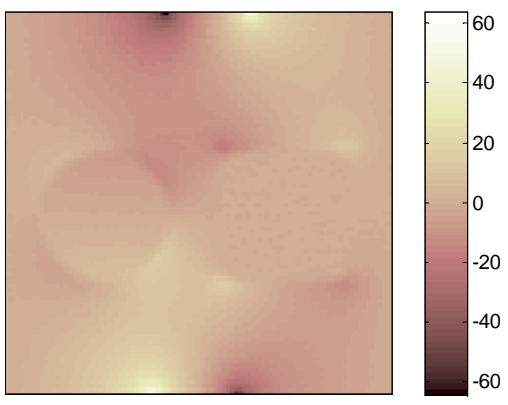

(d)

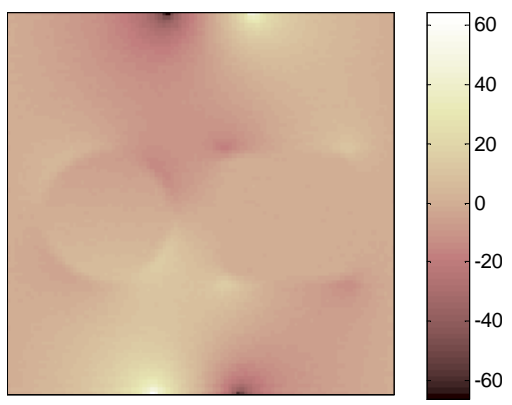

(f)

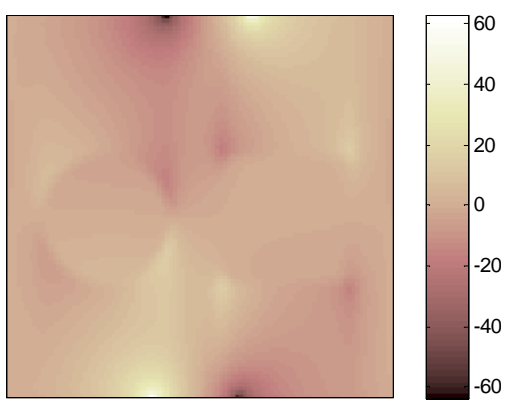

(c)

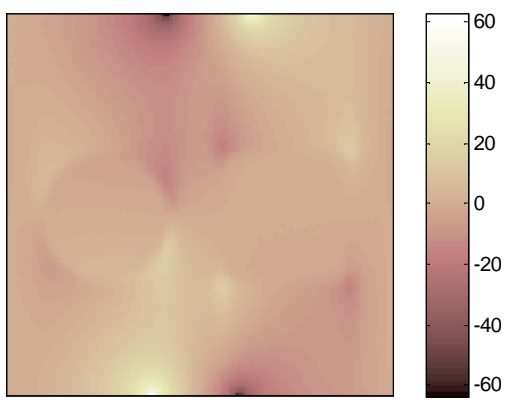

(e)

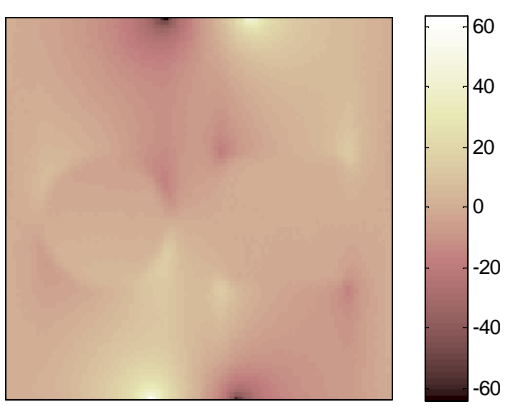

(g)

Figure 5.7 Reconstructed $J_{x}$ for different noise levels (vertical $20 \mathrm{~mA}$ current injection) a) Original $J_{x}\left(\mathrm{~A} / \mathrm{m}^{2}\right)$ distribution, b) Reconstructed $J_{x}\left(\mathrm{~A} / \mathrm{m}^{2}\right)$ using the proposed algorithm for noise-free case, c) Reconstructed $J_{x}\left(\mathrm{~A} / \mathrm{m}^{2}\right)$ using Iterative FT-MRCDI algorithm for noise-free case, d) Reconstructed $J_{x}\left(\mathrm{~A} / \mathrm{m}^{2}\right)$ using the proposed algorithm for SNR30 case, e) Reconstructed $J_{x}\left(\mathrm{~A} / \mathrm{m}^{2}\right)$ using Iterative FTMRCDI algorithm for SNR 30 case, f) Reconstructed $J_{x}\left(\mathrm{~A} / \mathrm{m}^{2}\right)$ using the proposed algorithm for SNR13 case, g) Reconstructed $J_{x}\left(\mathrm{~A} / \mathrm{m}^{2}\right)$ using Iterative FT-MRCDI algorithm for SNR 13 case. 


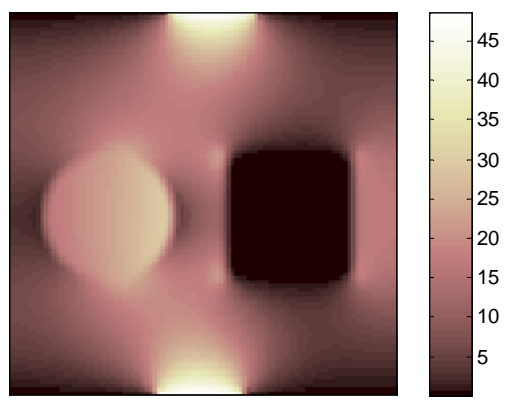

(a)

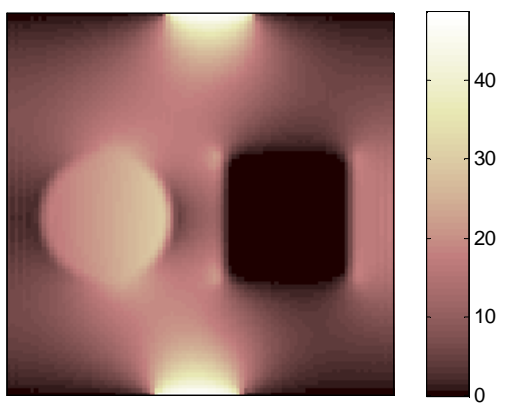

(b)

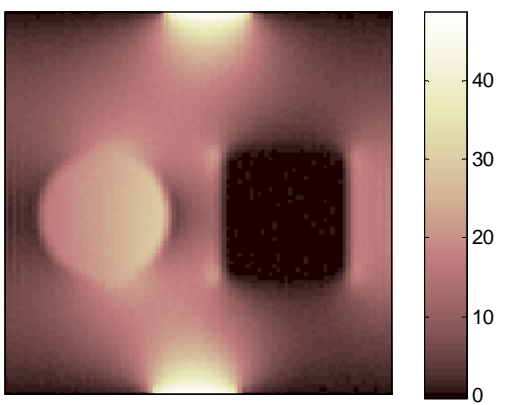

(d)

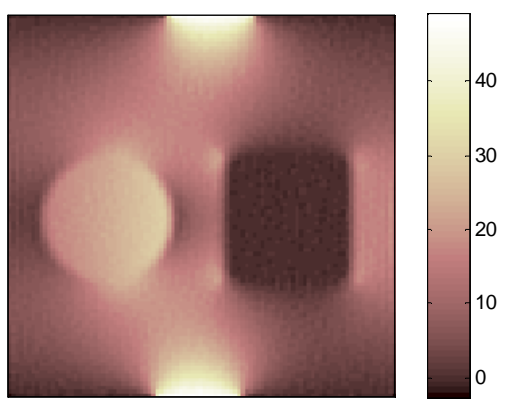

(f)

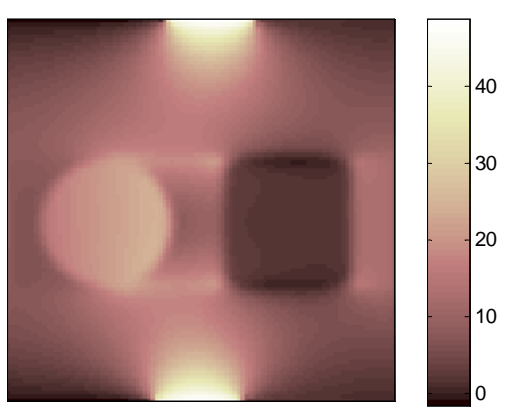

(c)

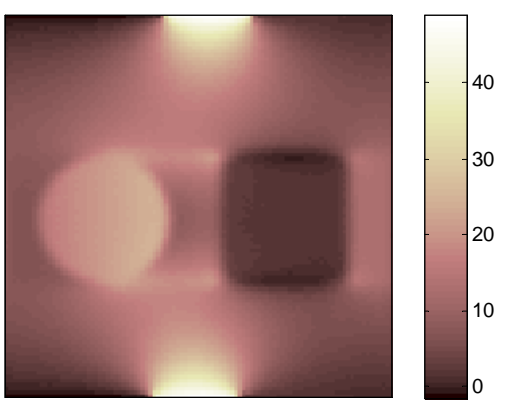

(e)

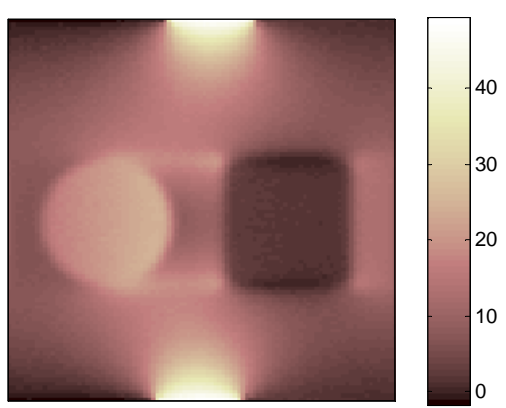

(g)

Figure 5.8 Reconstructed $J_{y}$ for different noise levels $(20 \mathrm{~mA}$ current is injected vertically and note that direction of positive y-axis is taken as negative) a) Original $J_{y}$ $\left(\mathrm{A} / \mathrm{m}^{2}\right)$ distribution, b) Reconstructed $J_{y}\left(\mathrm{~A} / \mathrm{m}^{2}\right)$ using the proposed algorithm for noise-free case, c) Reconstructed $J_{y}\left(\mathrm{~A} / \mathrm{m}^{2}\right)$ using Iterative FT-MRCDI algorithm for noise-free case, d) Reconstructed $J_{y}\left(\mathrm{~A} / \mathrm{m}^{2}\right)$ using the proposed algorithm for SNR30 case, e) Reconstructed $J_{y}\left(\mathrm{~A} / \mathrm{m}^{2}\right)$ using Iterative FT-MRCDI algorithm for SNR 30 case, f) Reconstructed $J_{y}\left(\mathrm{~A} / \mathrm{m}^{2}\right)$ using the proposed algorithm for SNR13 case, g) Reconstructed $J_{y}\left(\mathrm{~A} / \mathrm{m}^{2}\right)$ using Iterative FT-MRCDI algorithm for SNR 13 case. 
When the reconstruction performances of algorithms are compared by evaluating the reconstructed current density images perceptually, it is seen that the proposed algorithm is better in reconstructing current density for this model. As it can be seen from the above figures, some reconstruction artifacts occur at the corners of the square object in the reconstructed current density using the iterative FT-MRCDI algorithm. This is due to the assumption of solenoidal current flow. In reality, it is known that divergence of difference currents are zero. However, in simulations, the divergence of difference current density is not exactly zero in the regions where the conductivity does not change smoothly because of the discretization error. For this reason, undesired reconstruction artifacts may be introduced in the reconstructed image. These artifacts can be reduced by increasing the number of finite elements.

The quantitative evaluation of the reconstruction performances of the algorithms are listed in Table 5.2.

Table 5.2 Errors in the reconstructed current density for simulation model 2.

\begin{tabular}{|c|c|c|c|c|c|c|}
\hline \multirow{2}{*}{} & Horizontal Current Injection & \multicolumn{2}{c|}{ Vertical Current Injection } \\
\cline { 2 - 7 } & $J_{x}$ & $J_{y}$ & $J$ & $J_{x}$ & $J_{y}$ & $J$ \\
\hline \multicolumn{7}{|c|}{ The Proposed Algorithm } \\
\hline Noise-free & $0.34 \%$ & $1.34 \%$ & $0.69 \%$ & $1.25 \%$ & $0.89 \%$ & $0.86 \%$ \\
\hline SNR 30 & $1.11 \%$ & $2.14 \%$ & $1.18 \%$ & $2.49 \%$ & $1.49 \%$ & $1.40 \%$ \\
\hline SNR 13 & $2.48 \%$ & $3.80 \%$ & $2.19 \%$ & $4.98 \%$ & $2.85 \%$ & $2.60 \%$ \\
\hline \multicolumn{7}{|c|}{ Iterative FT-MRCDI Algorithm } \\
\hline Noise-free & $15.15 \%$ & $19.92 \%$ & $13.49 \%$ & $18.37 \%$ & $12.74 \%$ & $12.41 \%$ \\
\hline SNR 30 & $15.16 \%$ & $19.93 \%$ & $13.49 \%$ & $18.39 \%$ & $12.75 \%$ & $12.42 \%$ \\
\hline SNR 13 & $15.18 \%$ & $20.00 \%$ & $13.52 \%$ & $18.47 \%$ & $12.80 \%$ & $12.47 \%$ \\
\hline
\end{tabular}

Quantitative evaluation of the reconstruction performances of the algorithms shows that the proposed algorithm reconstructs with less error. It can be seen from Table 5.2 that the reconstruction performances of the algorithms for the component of current density orthogonal to the current injection pattern is poorer. 


\subsubsection{Results for Simulation Model 3}

The results obtained from simulation model 3 are presented in this subsection. The geometry and conductivity distribution of simulation model 3 was shown in Figure 4.3. Two noise levels, SNR 30 and SNR 13, were also used in these simulations.

Z-component of magnetic flux density, $B_{z}$, obtained from horizontal and vertical current injection patterns are shown in Figure 5.9.

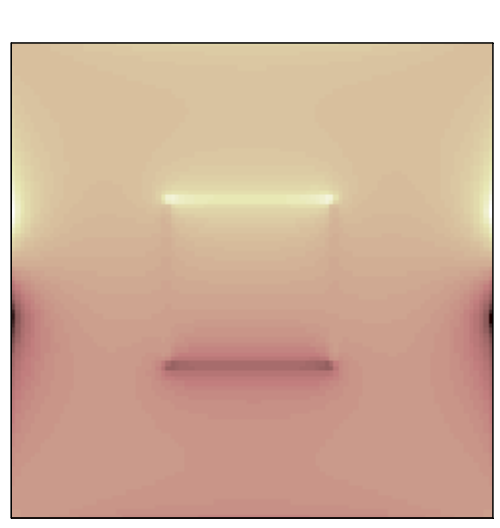

(a)

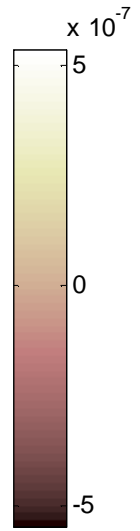
$-5$

a)

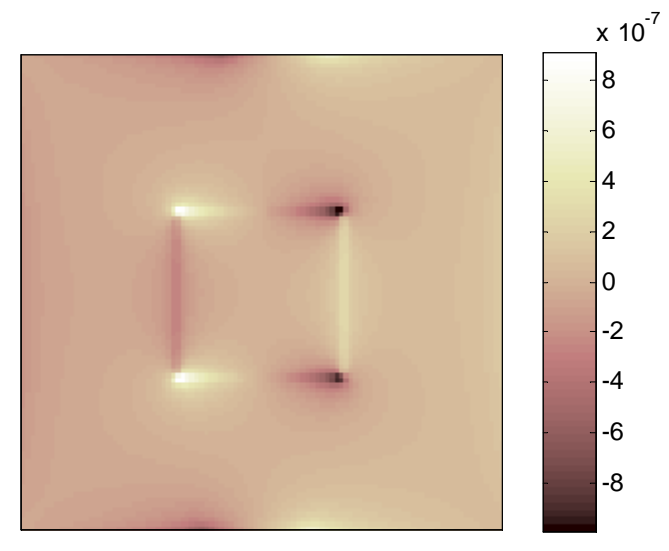

(b)

Figure 5.9 z-component of magnetic flux density, $B_{z}(\mathrm{~T})$ a) For horizontal current injection pattern, b) For vertical current injection pattern.

Reconstructed $J_{x}$ and $J_{y}$ for different noise levels for horizontal current injection pattern are shown in Figure 5.10 and Figure 5.11, respectively. For vertical current injection pattern, reconstructed $J_{x}$ and $J_{y}$ for different noise levels are shown in Figure 5.12 and Figure 5.13, respectively. Note that, the direction of positive y-axis is taken as negative in $J_{y}$ for vertical current injection to make the images consistent with $J_{x}$ images for horizontal current injection pattern. 


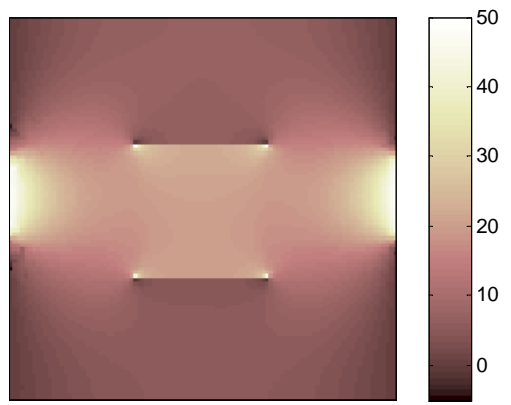

(a)

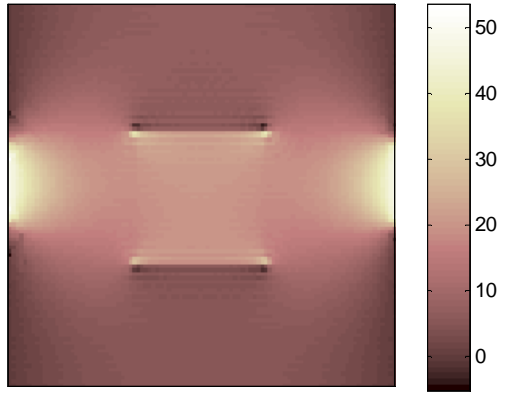

(b)

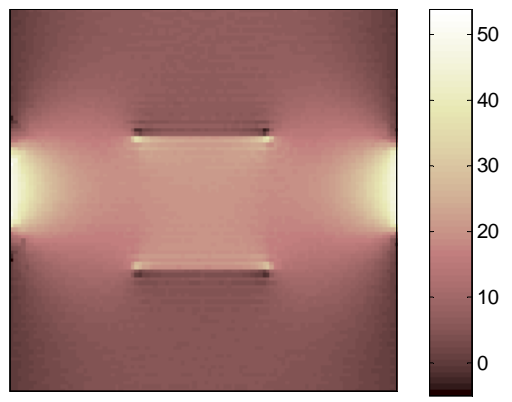

(d)

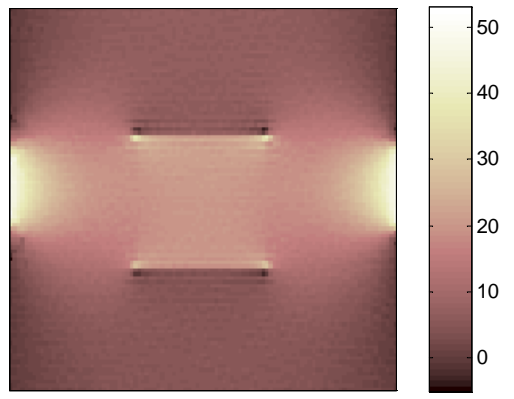

(f)

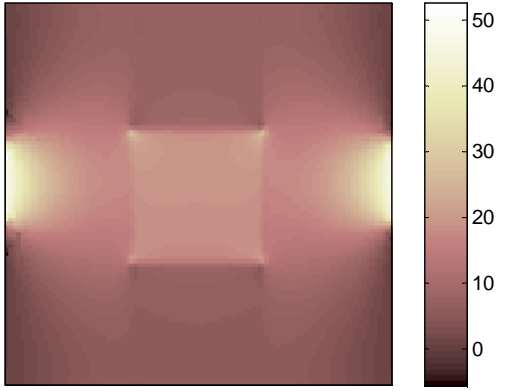

(c)

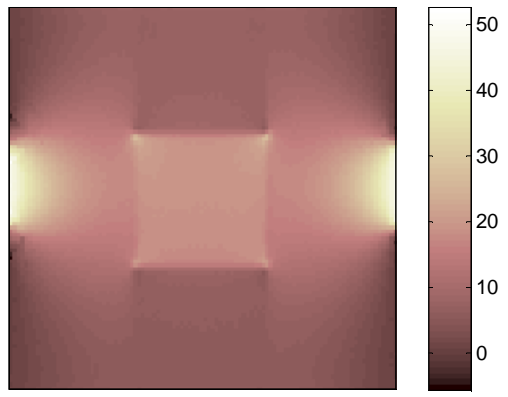

(e)

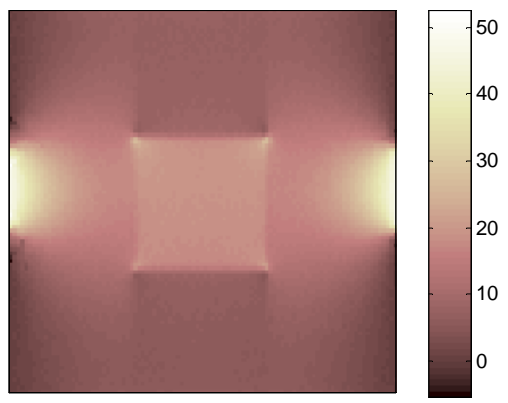

(g)

Figure 5.10 Reconstructed $J_{x}$ for different noise levels (horizontal $20 \mathrm{~mA}$ current injection) a) Original $J_{x}\left(\mathrm{~A} / \mathrm{m}^{2}\right)$ distribution, b) Reconstructed $J_{x}\left(\mathrm{~A} / \mathrm{m}^{2}\right)$ using the proposed algorithm for noise-free case, c) Reconstructed $J_{x}\left(\mathrm{~A} / \mathrm{m}^{2}\right)$ using Iterative FT-MRCDI algorithm for noise-free case, d) Reconstructed $J_{x}\left(\mathrm{~A} / \mathrm{m}^{2}\right)$ using the proposed algorithm for SNR30 case, e) Reconstructed $J_{x}\left(\mathrm{~A} / \mathrm{m}^{2}\right)$ using Iterative FTMRCDI algorithm for SNR 30 case, f) Reconstructed $J_{x}\left(\mathrm{~A} / \mathrm{m}^{2}\right)$ using the proposed algorithm for SNR13 case, g) Reconstructed $J_{x}\left(\mathrm{~A} / \mathrm{m}^{2}\right)$ using Iterative FT-MRCDI algorithm for SNR 13 case. 


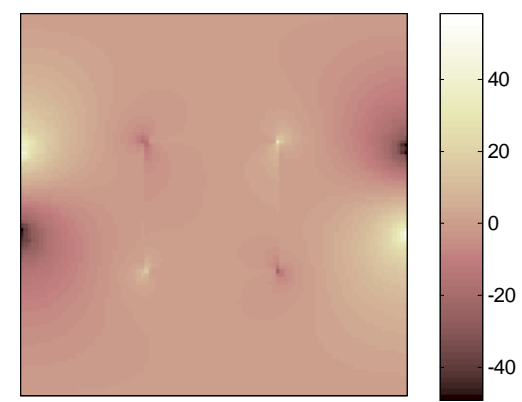

(a)

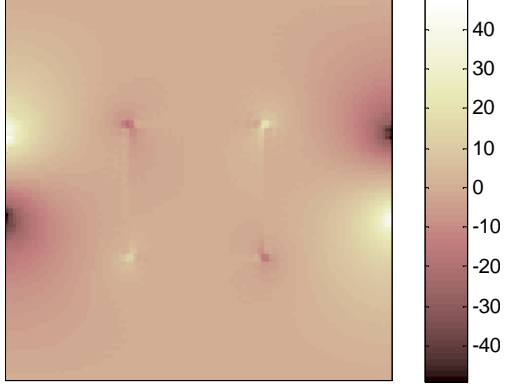

(b)

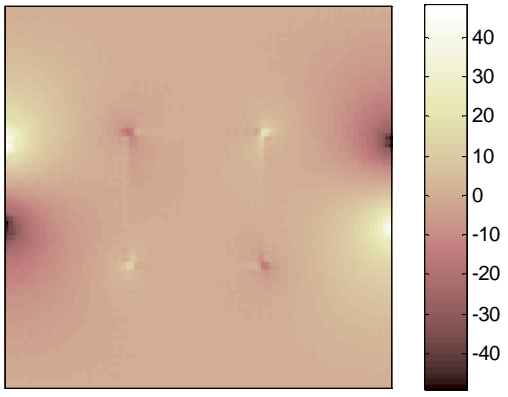

(d)

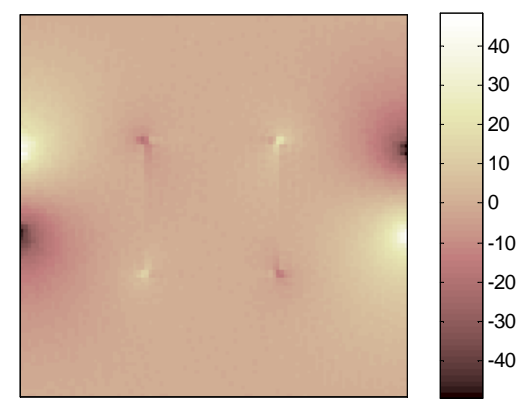

(f)

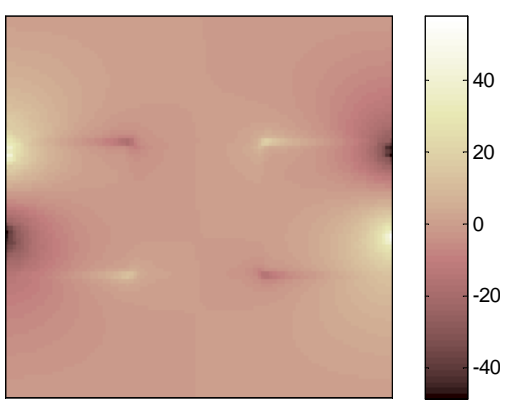

(c)

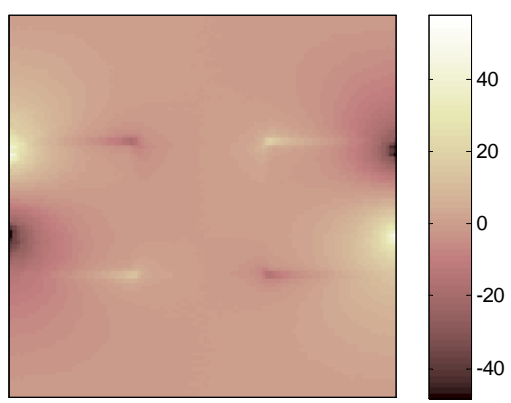

(e)

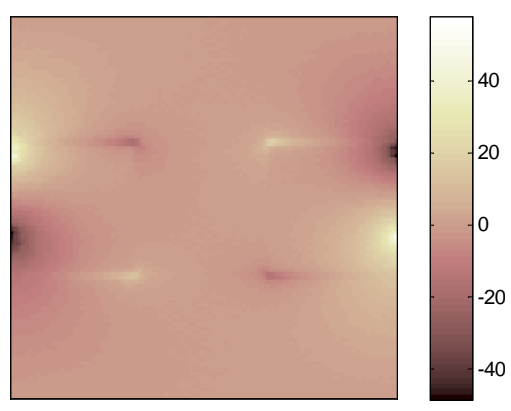

(g)

Figure 5.11 Reconstructed $J_{y}$ for different noise levels (horizontal $20 \mathrm{~mA}$ current injection) a) Original $J_{y}\left(\mathrm{~A} / \mathrm{m}^{2}\right)$ distribution, b) Reconstructed $J_{y}\left(\mathrm{~A} / \mathrm{m}^{2}\right)$ using the proposed algorithm for noise-free case, c) Reconstructed $J_{y}\left(\mathrm{~A} / \mathrm{m}^{2}\right)$ using Iterative FT-MRCDI algorithm for noise-free case, d) Reconstructed $J_{y}\left(\mathrm{~A} / \mathrm{m}^{2}\right)$ using the proposed algorithm for SNR30 case, e) Reconstructed $J_{y}\left(\mathrm{~A} / \mathrm{m}^{2}\right)$ using Iterative FTMRCDI algorithm for SNR 30 case, f) Reconstructed $J_{y}\left(\mathrm{~A} / \mathrm{m}^{2}\right)$ using the proposed algorithm for SNR13 case, g) Reconstructed $J_{y}\left(\mathrm{~A} / \mathrm{m}^{2}\right)$ using Iterative FT-MRCDI algorithm for SNR 13 case. 


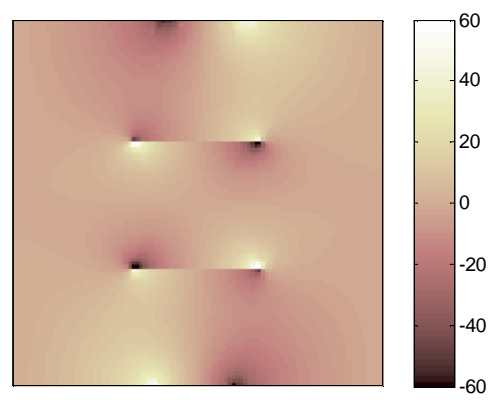

(a)

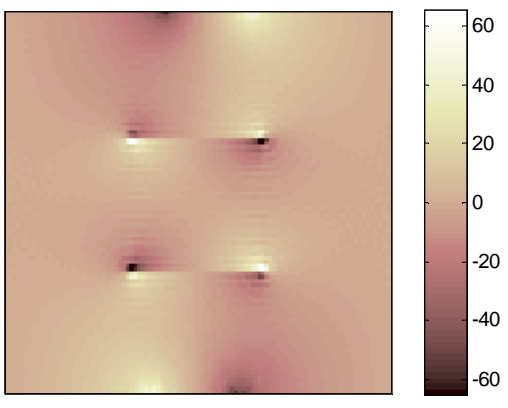

(b)

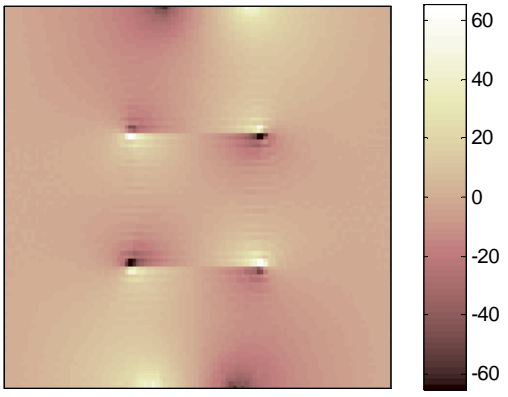

(d)

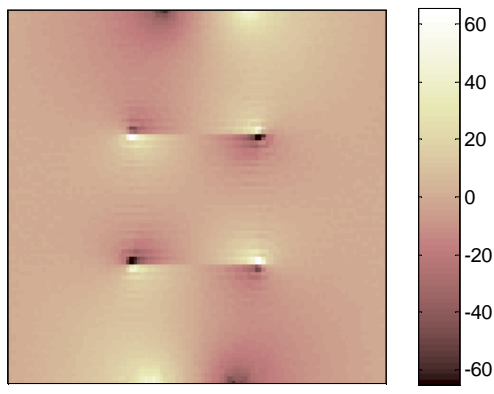

(f)

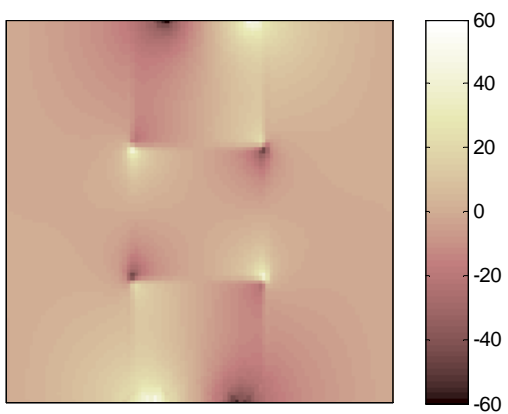

(c)

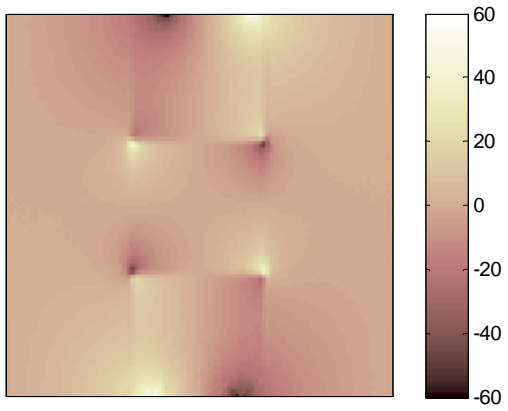

(e)

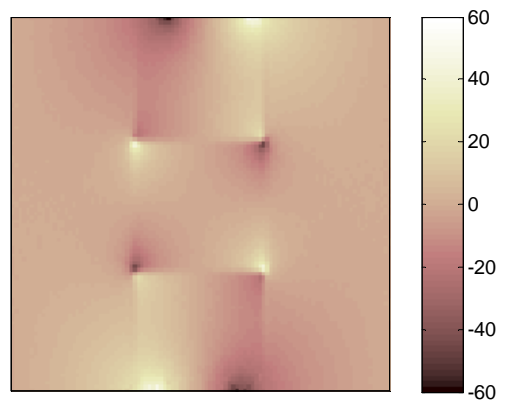

(g)

Figure 5.12 Reconstructed $J_{x}$ for different noise levels (vertical $20 \mathrm{~mA}$ current injection) a) Original $J_{x}\left(\mathrm{~A} / \mathrm{m}^{2}\right)$ distribution, b) Reconstructed $J_{x}\left(\mathrm{~A} / \mathrm{m}^{2}\right)$ using the proposed algorithm for noise-free case, c) Reconstructed $J_{x}\left(\mathrm{~A} / \mathrm{m}^{2}\right)$ using Iterative FT-MRCDI algorithm for noise-free case, d) Reconstructed $J_{x}\left(\mathrm{~A} / \mathrm{m}^{2}\right)$ using the proposed algorithm for SNR30 case, e) Reconstructed $J_{x}\left(\mathrm{~A} / \mathrm{m}^{2}\right)$ using Iterative FTMRCDI algorithm for SNR 30 case, f) Reconstructed $J_{x}\left(\mathrm{~A} / \mathrm{m}^{2}\right)$ using the proposed algorithm for SNR13 case, g) Reconstructed $J_{x}\left(\mathrm{~A} / \mathrm{m}^{2}\right)$ using Iterative FT-MRCDI algorithm for SNR 13 case. 


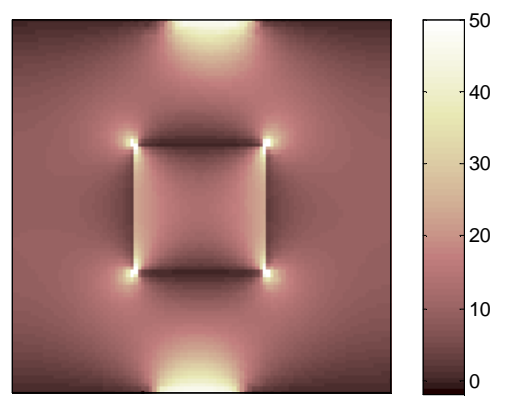

(a)

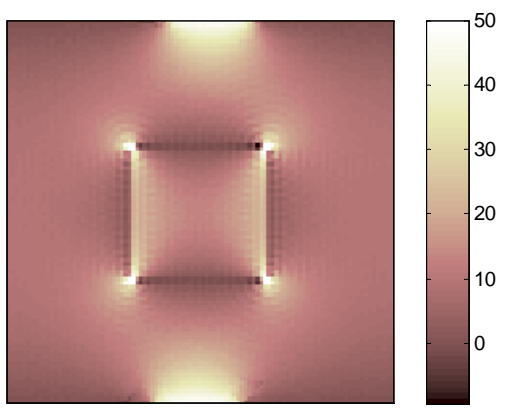

(b)

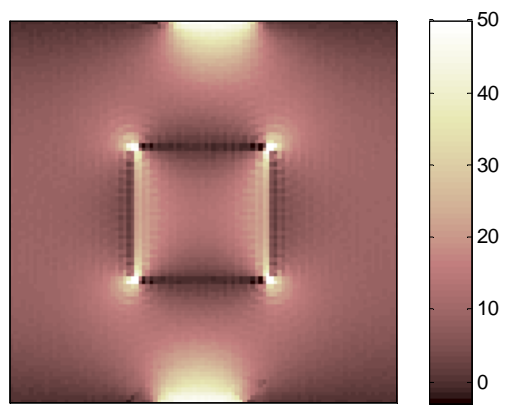

(d)

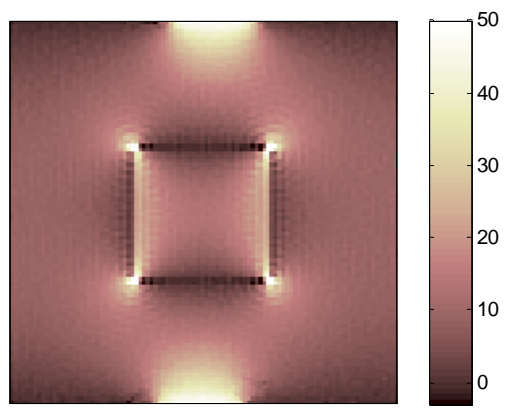

(f)

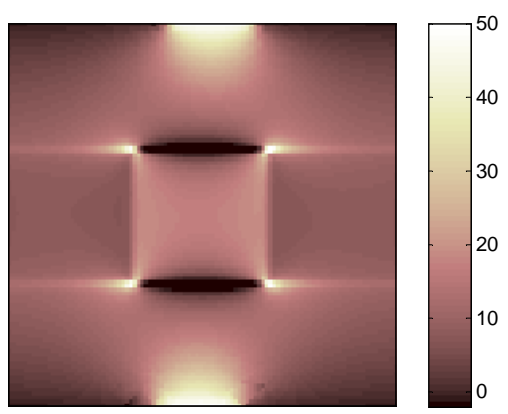

(c)

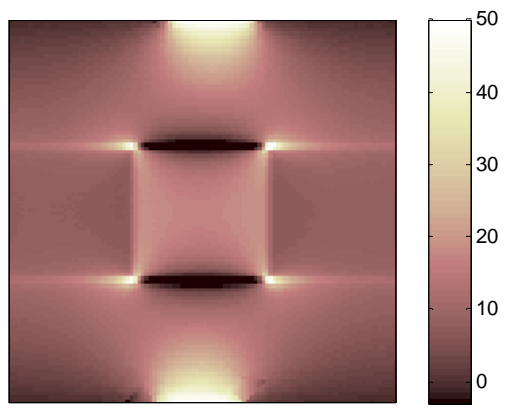

(e)

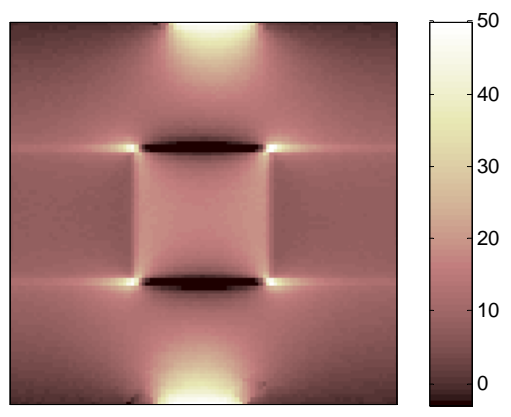

(g)

Figure 5.13 Reconstructed $J_{y}$ for different noise levels (20 mA current is injected vertically and note that direction of positive $y$-axis is taken as negative) a) Original $J_{y}$ $\left(\mathrm{A} / \mathrm{m}^{2}\right)$ distribution, b) Reconstructed $J_{y}\left(\mathrm{~A} / \mathrm{m}^{2}\right)$ using the proposed algorithm for noise-free case, c) Reconstructed $J_{y}\left(\mathrm{~A} / \mathrm{m}^{2}\right)$ using Iterative FT-MRCDI algorithm for noise-free case, d) Reconstructed $J_{y}\left(\mathrm{~A} / \mathrm{m}^{2}\right)$ using the proposed algorithm for SNR30 case, e) Reconstructed $J_{y}\left(\mathrm{~A} / \mathrm{m}^{2}\right)$ using Iterative FT-MRCDI algorithm for SNR 30 case, f) Reconstructed $J_{y}\left(\mathrm{~A} / \mathrm{m}^{2}\right)$ using the proposed algorithm for SNR13 case, g) Reconstructed $J_{y}\left(\mathrm{~A} / \mathrm{m}^{2}\right)$ using Iterative FT-MRCDI algorithm for SNR 13 case. 
When the reconstruction performances of algorithms are compared by evaluating the reconstructed current density images perceptually, it is seen that both algorithms show similar reconstruction performances for this model. In Figure 5.13, there are reconstruction artifacts in the images reconstructed by using the proposed algorithm. In other figures, these artifacts are relatively small. In the iterative FT-MRCDI algorithm, the reconstructed images have lower resolutions due to the low pass filter. This filtering effect is more significant on the edges.

The quantitative evaluation of the reconstruction performances of the algorithms are listed in Table 5.3.

Table 5.3 Errors in the reconstructed current density for simulation model 3.

\begin{tabular}{|c|c|c|c|c|c|c|}
\hline \multirow{2}{*}{} & \multicolumn{7}{|c|}{ Horizontal Current Injection } & \multicolumn{2}{c|}{ Vertical Current Injection } \\
\cline { 2 - 7 } & $J_{x}$ & $J_{y}$ & $J$ & $J_{x}$ & $J_{y}$ & $J$ \\
\hline The Proposed Algorithm \\
\hline Noise-free & $4.41 \%$ & $5.11 \%$ & $3.96 \%$ & $21.03 \%$ & $8.76 \%$ & $11.68 \%$ \\
\hline SNR 30 & $4.54 \%$ & $5.65 \%$ & $4.08 \%$ & $21.10 \%$ & $8.83 \%$ & $11.71 \%$ \\
\hline SNR 13 & $5.07 \%$ & $7.69 \%$ & $4.60 \%$ & $21.37 \%$ & $9.22 \%$ & $11.92 \%$ \\
\hline \multicolumn{7}{|c|}{ Iterative FT-MRCDI Algorithm } \\
\hline Noise-free & $10.97 \%$ & $15.05 \%$ & $10.18 \%$ & $37.43 \%$ & $19.96 \%$ & $22.71 \%$ \\
\hline SNR 30 & $10.99 \%$ & $15.10 \%$ & $10.20 \%$ & $37.44 \%$ & $19.97 \%$ & $22.72 \%$ \\
\hline SNR 13 & $11.04 \%$ & $15.24 \%$ & $10.25 \%$ & $37.45 \%$ & $19.99 \%$ & $22.71 \%$ \\
\hline
\end{tabular}

Quantitative evaluation of the reconstruction performances of the algorithms shows that the proposed algorithm reconstructs with less error for this simulation model.

\subsection{Experimental Results}

\subsubsection{Results for Experimental Model 1}

The results obtained from experimental model 1 are presented in this subsection. The experimental model 1 and its MR magnitude image were given in Figure 4.6. The 
experimental procedure mentioned in Section 2.5 is followed during this experiment. The $\mathrm{z}$ component of magnetic flux density for horizontal and vertical current injection patterns are shown in Figure 5.14.

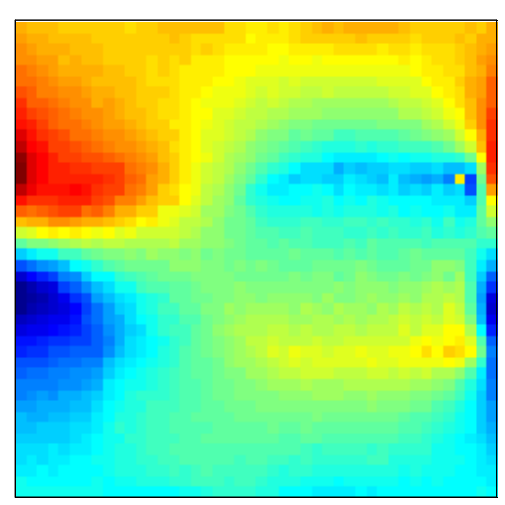

(a)
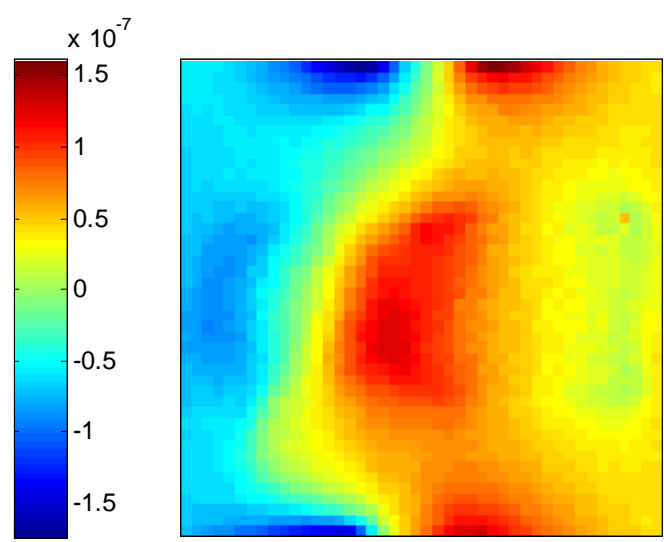

(b)

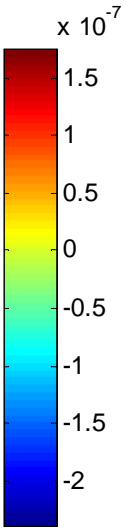

Figure 5.14 Magnetic flux density in z direction, $B_{z}(\mathrm{~T})$, a) For horizontal current injection pattern, b) For vertical current injection pattern.

In the experiments, other components of the magnetic flux density, $B_{x}$ and $B_{y}$, were also measured and the classical MRCDI algorithm using three components of $\vec{B}$ is used to calculate $\vec{J}$ as well. Therefore, the algorithms using three components of $\vec{B}$ and one component of $\vec{B}$ can be compared.

The results of experimental model 1 for horizontal and vertical current injection patterns are shown in Figure 5.15 and Figure 5.16, respectively. Note that, the direction of positive $y$-axis is taken as negative in $J_{y}$ for vertical current injection to make the images consistent with $J_{x}$ images for horizontal current injection pattern. 


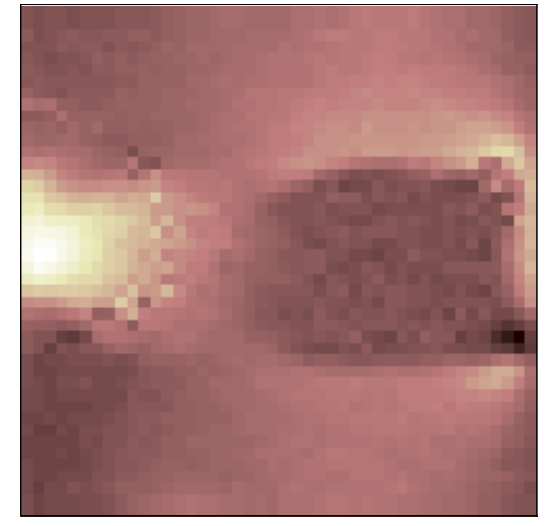

(a)

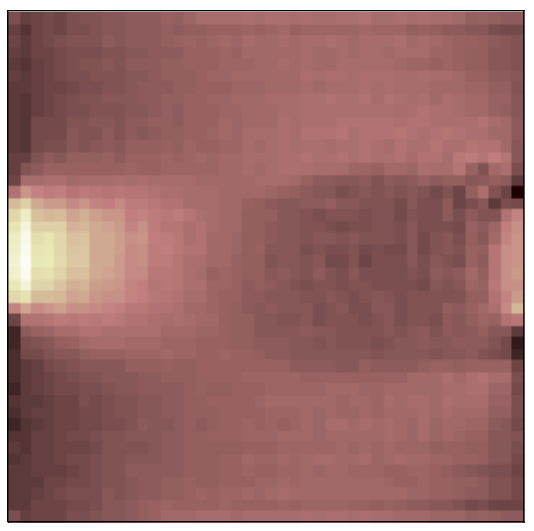

(c)

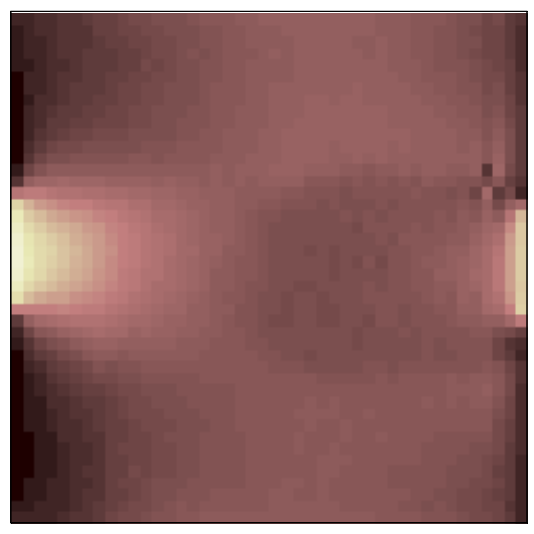

(e)
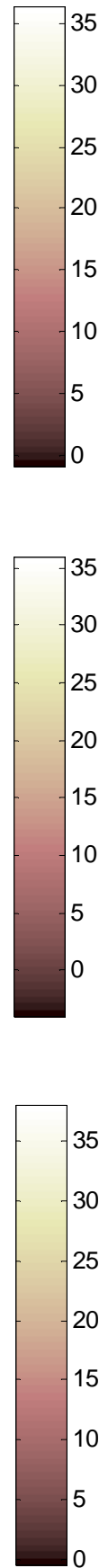

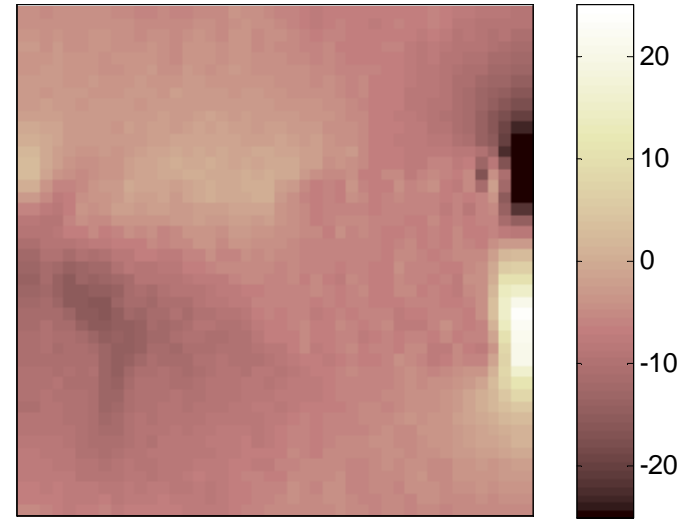

(b)

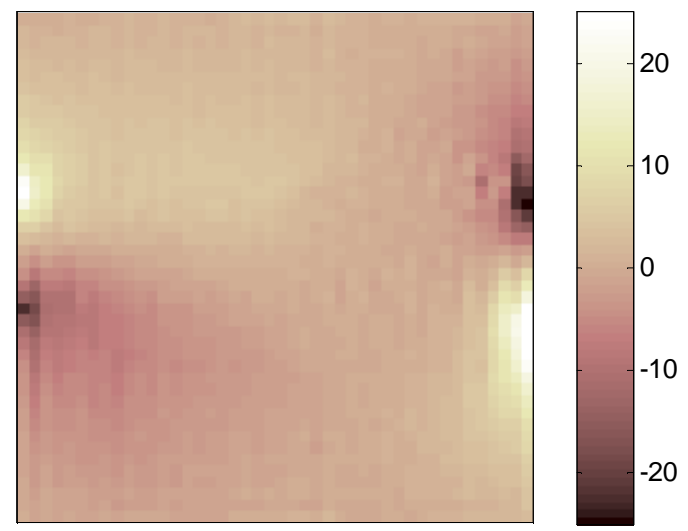

(d)

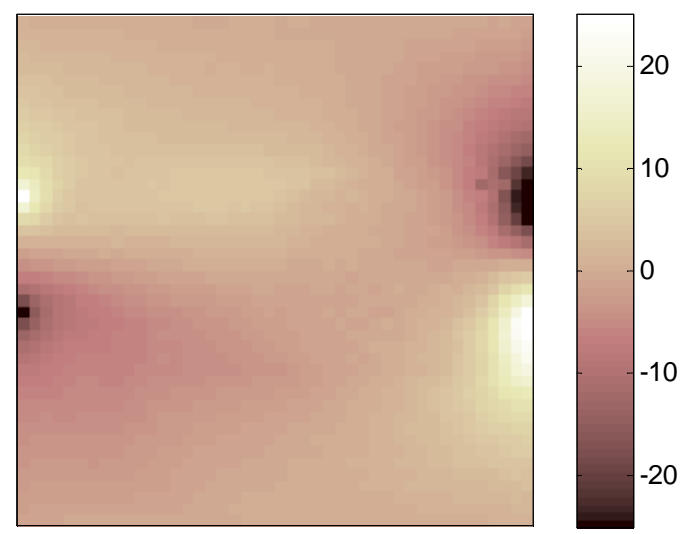

(f)

Figure 5.15 Results for horizontal current injection pattern, a) Reconstructed $J_{x}$ $\left(\mathrm{A} / \mathrm{m}^{2}\right)$ using three components of $\vec{B}$, b) Reconstructed $J_{y}\left(\mathrm{~A} / \mathrm{m}^{2}\right)$ using three components of $\vec{B}$, c) Reconstructed $J_{x}\left(\mathrm{~A} / \mathrm{m}^{2}\right)$ using the proposed algorithm, d) Reconstructed $J_{y}\left(\mathrm{~A} / \mathrm{m}^{2}\right)$ using the proposed algorithm, e) Reconstructed $J_{x}\left(\mathrm{~A} / \mathrm{m}^{2}\right)$ using Iterative FT-MRCDI algorithm, f) Reconstructed $J_{y}\left(\mathrm{~A} / \mathrm{m}^{2}\right)$ using Iterative FTMRCDI algorithm. 


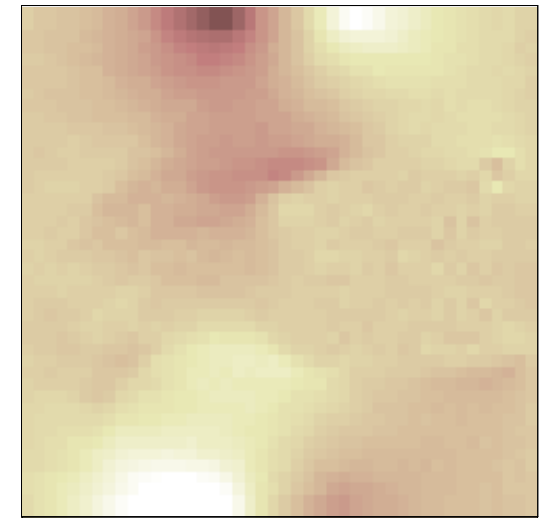

(a)

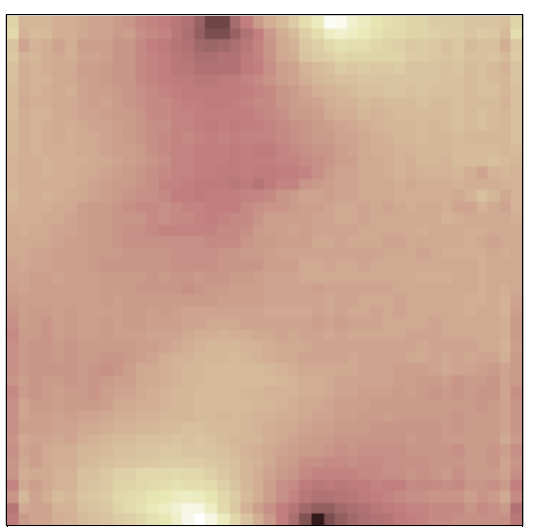

(c)

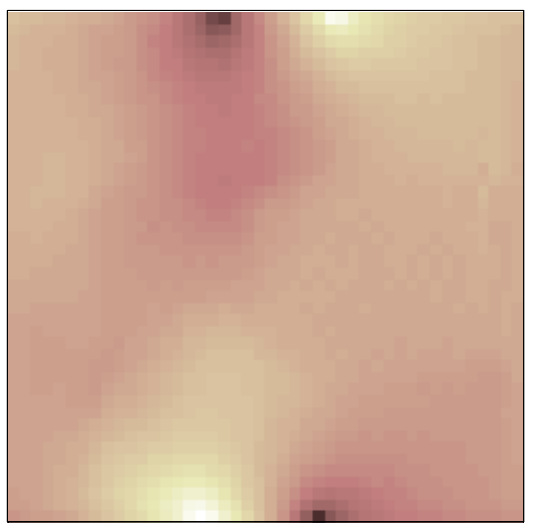

(e)
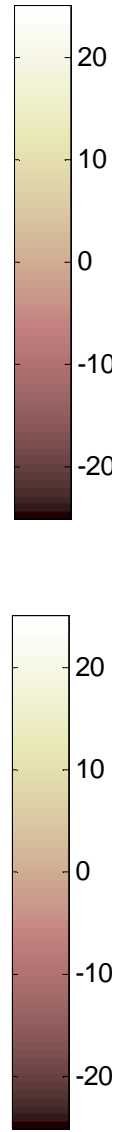

20

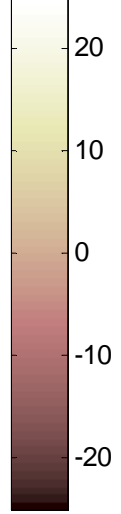

$-20$

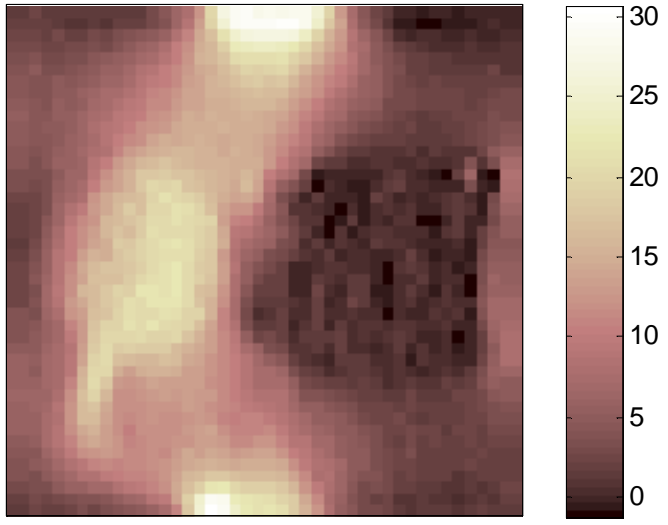

(b)

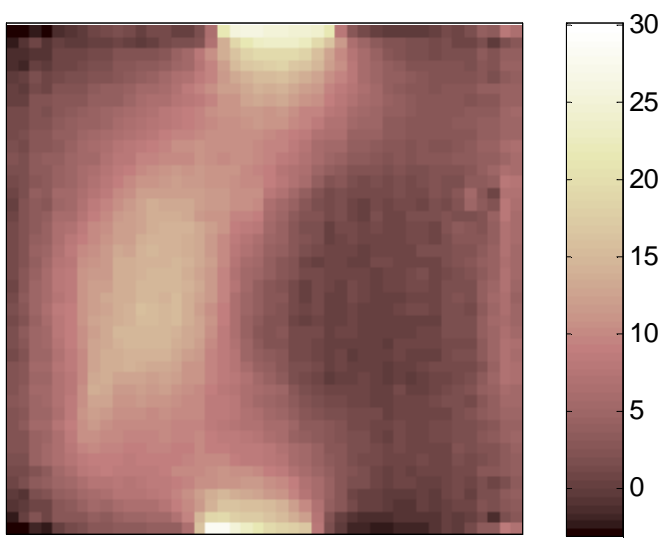

(d)

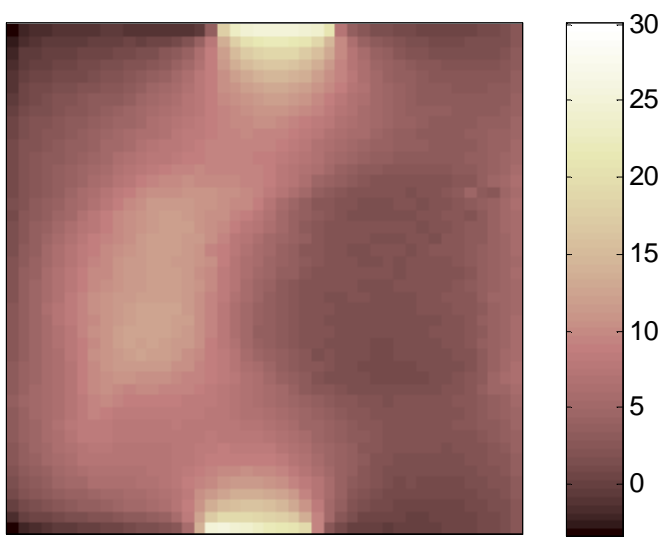

(f)

Figure 5.16 Results for vertical current injection pattern, (note that direction of positive $\mathrm{y}$-axis is taken as negative) a) Reconstructed $J_{x}(\mathrm{~A} / \mathrm{m} 2)$ using three components of $\vec{B}, \mathrm{~b})$ Reconstructed $J_{y}(\mathrm{~A} / \mathrm{m} 2)$ using three components of $\vec{B}$, c) Reconstructed $J_{x}(\mathrm{~A} / \mathrm{m} 2)$ using the proposed algorithm, d) Reconstructed $J_{y}(\mathrm{~A} / \mathrm{m} 2)$ using the proposed algorithm, e) Reconstructed $J_{x}(\mathrm{~A} / \mathrm{m} 2)$ using Iterative FT-MRCDI algorithm, f) Reconstructed $J_{y}(\mathrm{~A} / \mathrm{m} 2)$ using Iterative FT-MRCDI algorithm. 
The results of experimental model 1 are evaluated in three different aspects. First of all, the reconstructed images are evaluated perceptually. From Figure 5.15 and Figure 5.16, it can be seen that the classical MRCDI algorithm using three components of $\vec{B}$ reconstructs $J_{x}$ and $J_{y}$ with higher resolution. One of the reasons behind this situation is that low pass filter is applied in the proposed algorithm and in the iterative FT-MRCDI algorithm. This low pass filtering effect reduces the resolution.

The reconstruction performance of the proposed algorithm seems a little bit better than the iterative FT-MRCDI algorithms. Both algorithms reconstruct $J_{x}$ for horizontal current injection pattern and $J_{y}$ for vertical current injection pattern well. However, the performances of these algorithms in reconstructing $J_{x}$ for vertical current injection pattern and $J_{y}$ for horizontal current injection pattern are poor.

Secondly, the reconstructed images are verified by using divergence theorem. The details of this verification method were mentioned in Section 4.5.1. Calculated standard deviations are listed in Table 5.4. In this table, $\sigma_{1}$ is the standard deviation of the classical MRCDI algorithm which uses three components of $\vec{B}, \sigma_{2}$ is the standard deviation of the proposed algorithm and $\sigma_{3}$ is the standard deviation of the iterative FT-MRCDI algorithm.

Table 5.4 Calculated standard deviations for experimental model 1.

\begin{tabular}{|c|c|c|}
\hline & $J_{x}$ & $J_{y}$ \\
(horizontal current injection) & (vertical current injection) \\
\hline$\sigma_{1}$ & $11 \times 10^{-4}$ & $8 \times 10^{-4}$ \\
\hline$\sigma_{2}$ & $8.77 \times 10^{-4}$ & $5.04 \times 10^{-4}$ \\
\hline$\sigma_{3}$ & $3.2 \times 10^{-4}$ & $1.94 \times 10^{-4}$ \\
\hline
\end{tabular}

As seen in Table 5.4, the iterative FT-MRCDI algorithm reconstructs the current density distribution with the smallest standard deviation. It can be said that the 
iterative FT-MRCDI algorithm reconstructs the most consistent current density images since the deviations of the integrals of $\vec{J}$ in each row is small.

Finally, the reconstructed images are evaluated in terms of the reconstructed MREIT conductivities. Hence, J-substitution algorithm is used to reconstruct conductivity distribution from the reconstructed $\vec{J}$. The reconstructed conductivities are shown in Figure 5.17. Note that the J-substitution algorithm is stopped after 5 iterations.

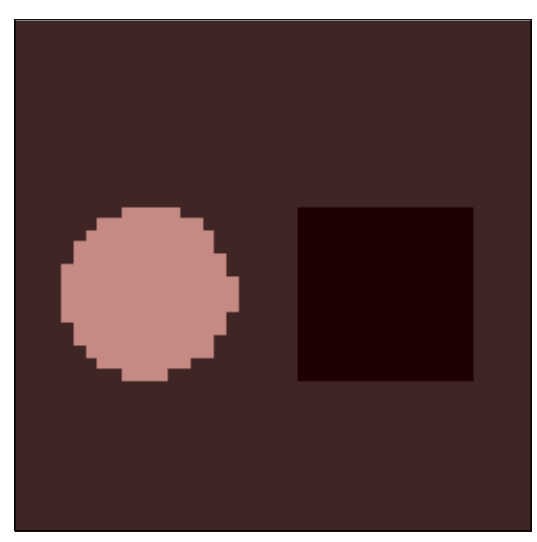

(a)

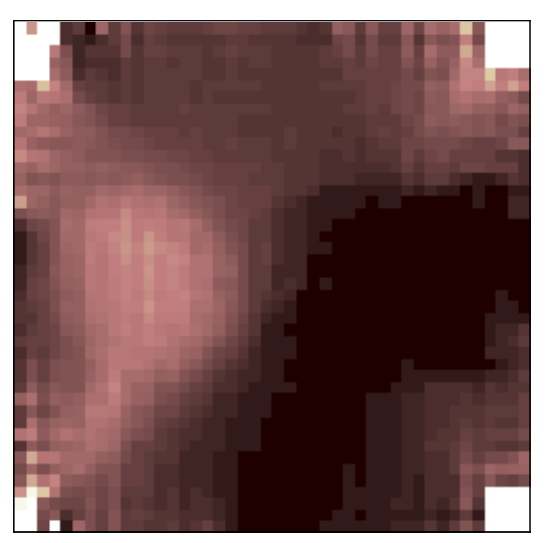

(c)
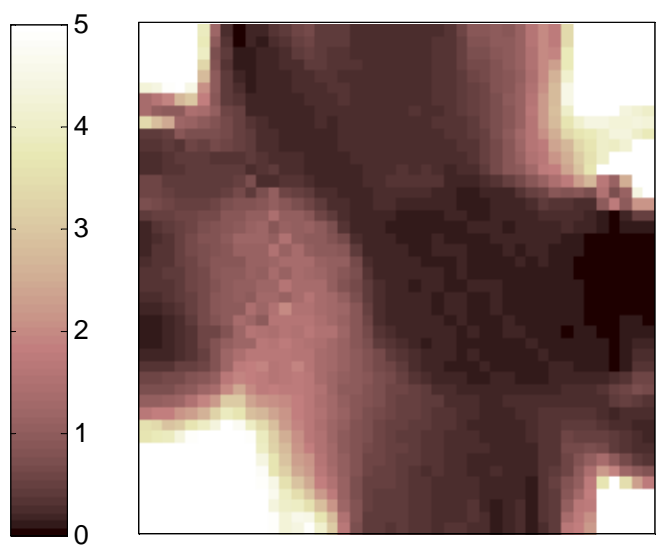

(b)
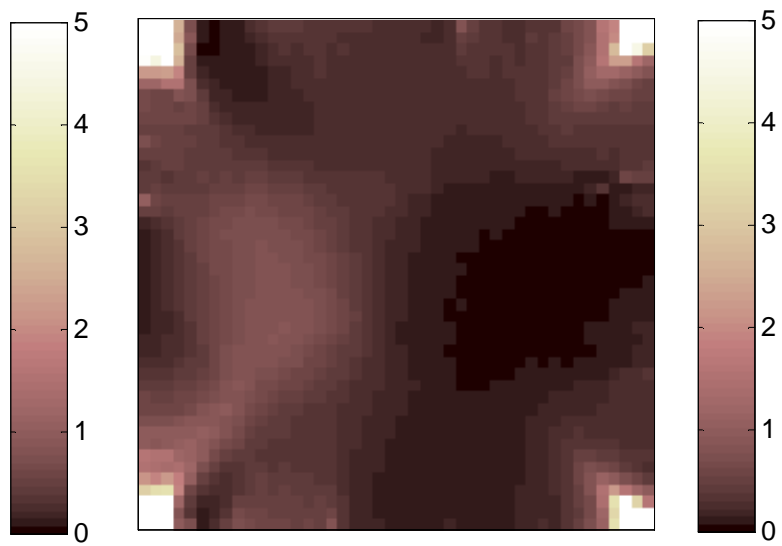

(d)

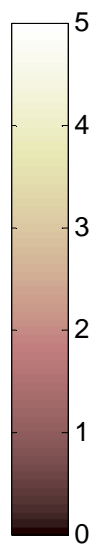

Figure 5.17 Reconstructed conductivity images with J-substitution algorithm after 5 iterations, a) Original distribution, b) Reconstructed conductivity image using $\vec{J}$ obtained from the classical MRCDI algorithm, c) Reconstructed conductivity image using $\vec{J}$ obtained from the proposed algorithm, d) Reconstructed conductivity image using $\vec{J}$ obtained from the iterative FT-MRCDI algorithm.

Evaluating the performances of the current density reconstruction algorithms in 
terms of error in the reconstructed conductivity images, is not possible due to the reconstruction artifacts at the corners of the field of view (FOV). The reconstructed conductivity values of these corners are dominating the conductivity of the remaining regions. Therefore, the reconstructed conductivity images are only evaluated perceptually. The J-substitution algorithm seems to reconstruct the best conductivity image by using $\vec{J}$ obtained from the proposed algorithm. The circular object can be clearly distinguished and it is reconstructed with $26.3 \%$ error. The circular object can be distinguished in Figure 5.17b and Figure 5.17d as well. In Figure 5.17b, it is reconstructed with $43.5 \%$ error and in Figure 5.17 d, it is reconstructed with $65.6 \%$ error.

\subsubsection{Results for Experimental Model 2}

The results obtained from experimental model 2 are presented in this subsection. The experimental model 2 and its MR magnitude image were given in Figure 4.7. The $\mathrm{z}$ component of magnetic flux density for horizontal and vertical current injection patterns are shown in Figure 5.18.

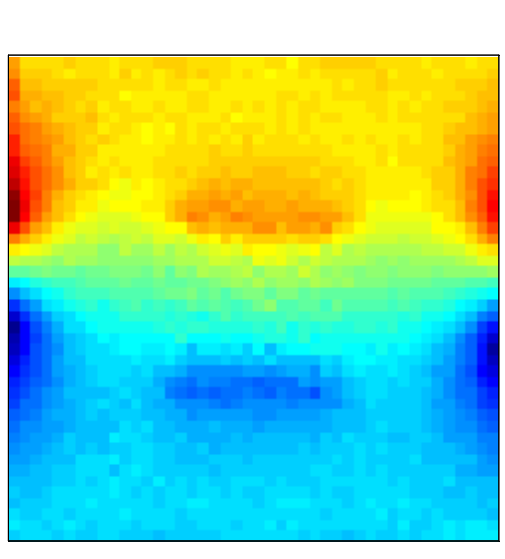

(a)
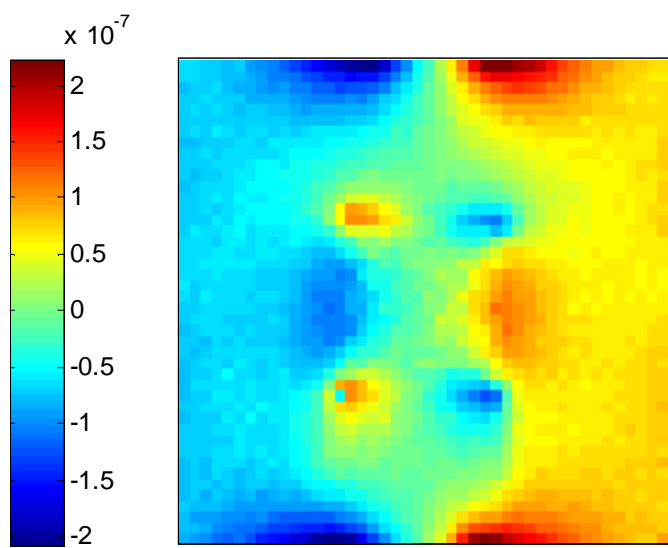

(b)

Figure 5.18 Magnetic flux density in z direction, $B_{z}(\mathrm{~T})$, a) For horizontal current injection pattern, b) For vertical current injection pattern.

The results of experimental model 2 for horizontal and vertical current injection patterns are shown in Figure 5.19 and Figure 5.20, respectively. Note that, the direction of positive $y$-axis is taken as negative in $J_{y}$ for vertical current injection to make the images consistent with $J_{x}$ images for horizontal current injection pattern. 


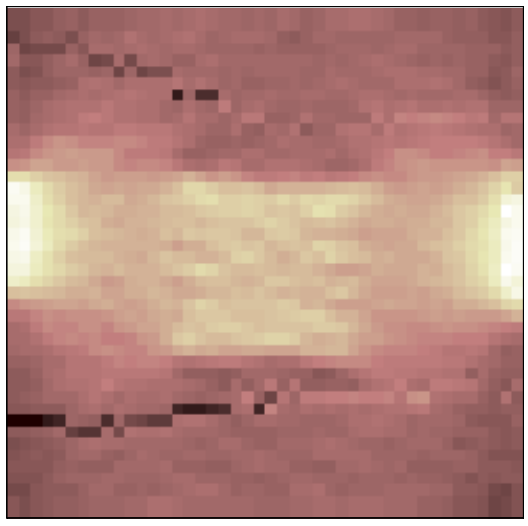

(a)

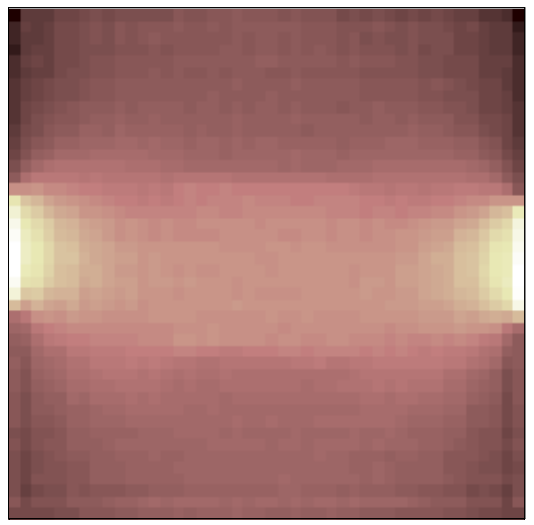

(c)

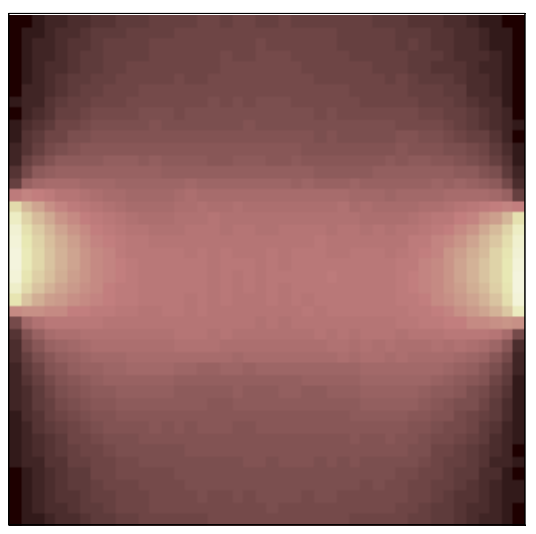

(e)
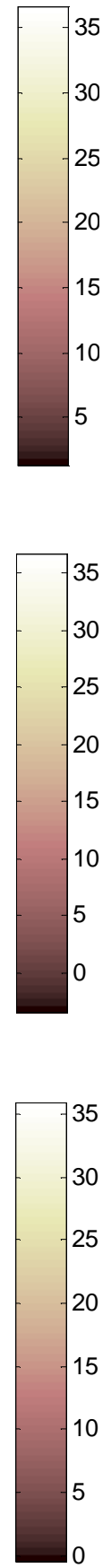

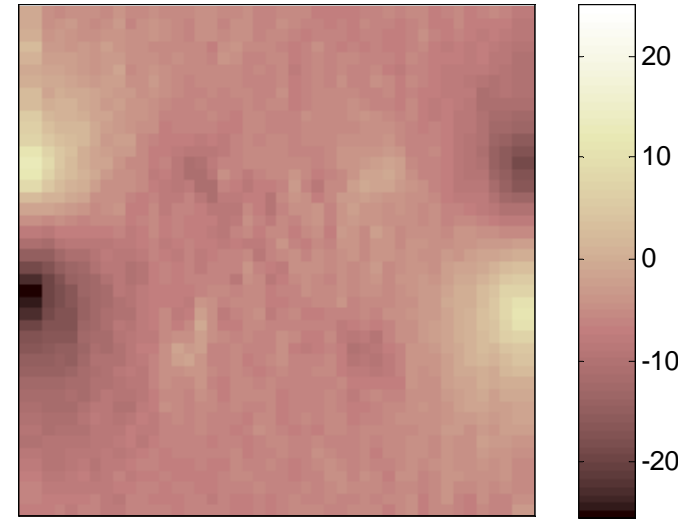

(b)

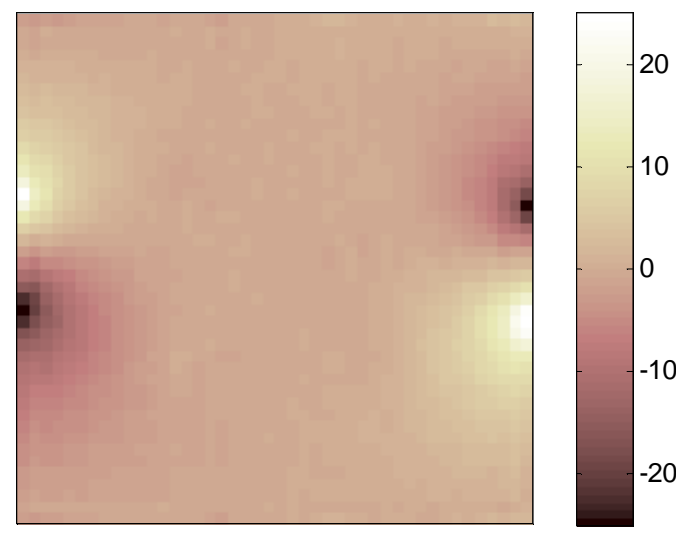

(d)

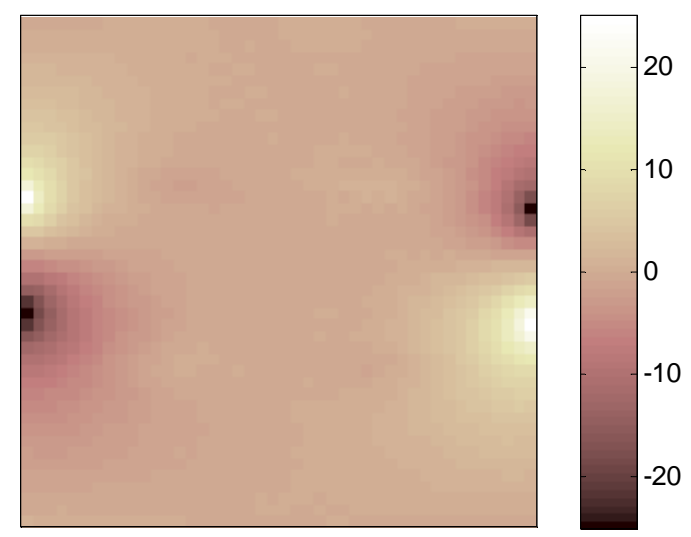

(f)

Figure 5.19 Results for horizontal current injection pattern, a) Reconstructed $J_{x}$ $\left(\mathrm{A} / \mathrm{m}^{2}\right)$ using three components of $\vec{B}$, b) Reconstructed $J_{y}\left(\mathrm{~A} / \mathrm{m}^{2}\right)$ using three components of $\vec{B}$, c) Reconstructed $J_{x}\left(\mathrm{~A} / \mathrm{m}^{2}\right)$ using the proposed algorithm, d) Reconstructed $J_{y}\left(\mathrm{~A} / \mathrm{m}^{2}\right)$ using the proposed algorithm, e) Reconstructed $J_{x}\left(\mathrm{~A} / \mathrm{m}^{2}\right)$ using Iterative FT-MRCDI algorithm, f) Reconstructed $J_{y}\left(\mathrm{~A} / \mathrm{m}^{2}\right)$ using Iterative FTMRCDI algorithm. 


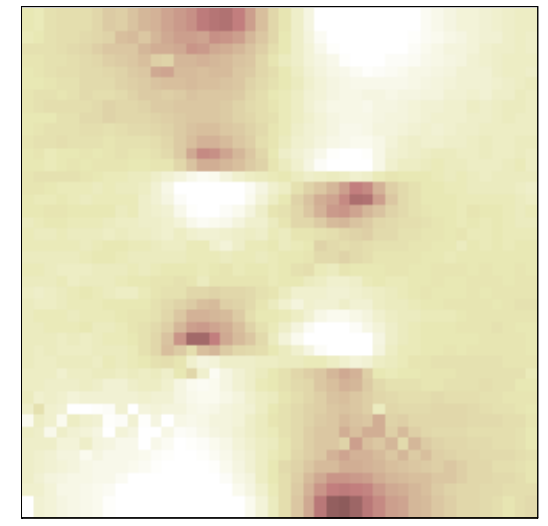

(a)

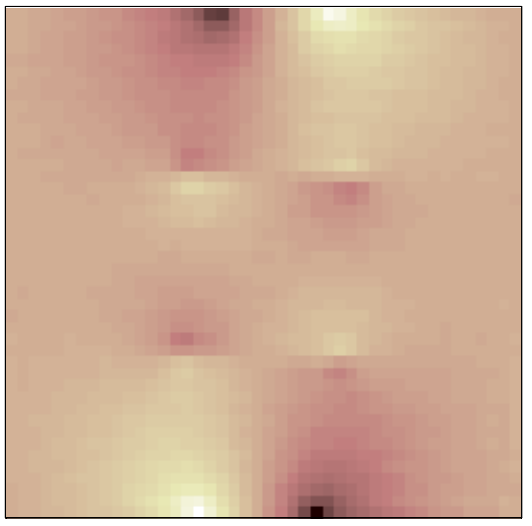

(c)

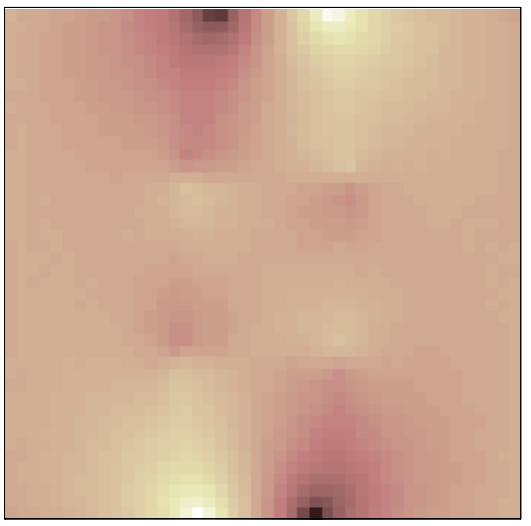

(e)
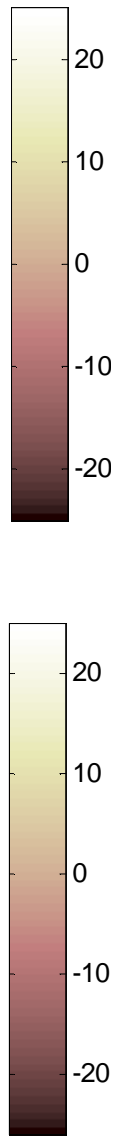

0

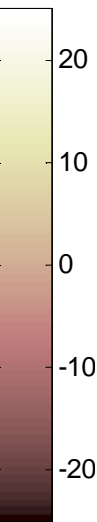

20
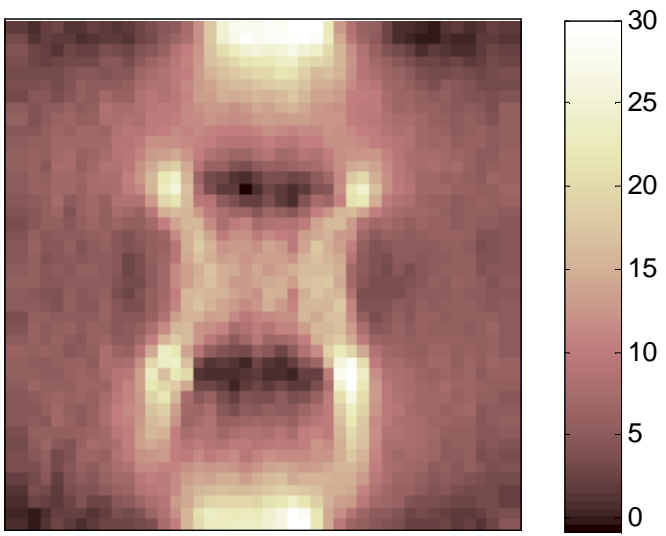

(b)

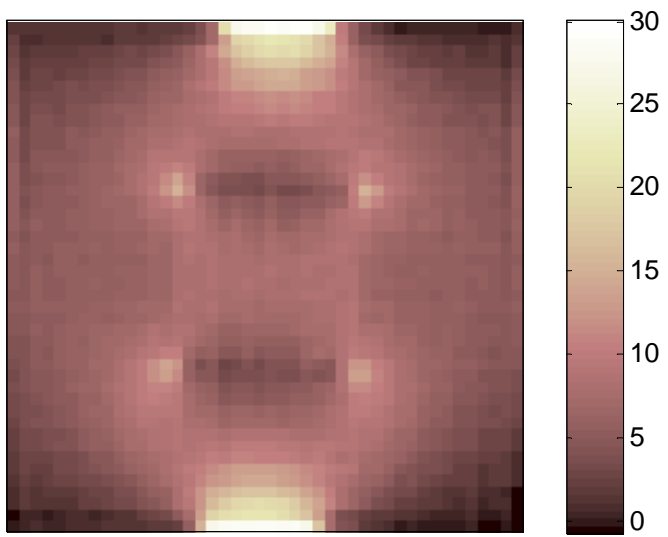

(d)

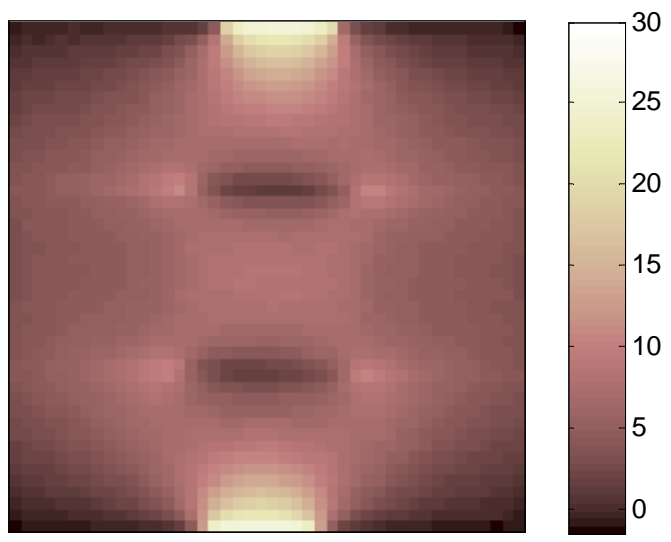

(f)

Figure 5.20 Results for vertical current injection pattern, (note that direction of positive $\mathrm{y}$-axis is taken as negative) a) Reconstructed $J_{x}\left(\mathrm{~A} / \mathrm{m}^{2}\right)$ using three components of $\vec{B}$, b) Reconstructed $J_{y}\left(\mathrm{~A} / \mathrm{m}^{2}\right)$ using three components of $\vec{B}$, c) Reconstructed $J_{x}\left(\mathrm{~A} / \mathrm{m}^{2}\right)$ using the proposed algorithm, d) Reconstructed $J_{y}\left(\mathrm{~A} / \mathrm{m}^{2}\right)$ using the proposed algorithm, e) Reconstructed $J_{x}\left(\mathrm{~A} / \mathrm{m}^{2}\right)$ using Iterative FT-MRCDI algorithm, f) Reconstructed $J_{y}\left(\mathrm{~A} / \mathrm{m}^{2}\right)$ using Iterative FT-MRCDI algorithm. 
When reconstruction performances of the algorithms are compared by evaluating the reconstructed current density images perceptually, it is seen that the classical MRCDI algorithm has the best performance. The current paths can be seen in detail in the reconstructed current density image obtained by using this algorithm. The reconstruction performances of the proposed algorithm and the iterative FT-MRCDI algorithm are similar.

The reconstructed images are also verified by using divergence theorem. Calculated standard deviations are listed in Table 5.5. In this table, $\sigma_{1}$ is the standard deviation of the classical MRCDI algorithm which uses three components of $\vec{B}, \sigma_{2}$ is the standard deviation of the proposed algorithm and $\sigma_{3}$ is the standard deviation of the iterative FT-MRCDI algorithm.

Table 5.5 Calculated standard deviations for experimental model 2.

\begin{tabular}{|c|c|c|}
\hline & $J_{x}$ & $J_{y}$ \\
(horizontal current injection) & (vertical current injection) \\
\hline$\sigma_{1}$ & $6.86 \times 10^{-4}$ & $7.06 \times 10^{-4}$ \\
\hline$\sigma_{2}$ & $4.58 \times 10^{-4}$ & $4.53 \times 10^{-4}$ \\
\hline$\sigma_{3}$ & $1.52 \times 10^{-4}$ & $1.32 \times 10^{-4}$ \\
\hline
\end{tabular}

As seen in Table 5.5, the iterative FT-MRCDI algorithm reconstructs the current density distribution with the smallest standard deviation. It can be said that the iterative FT-MRCDI algorithm reconstructs the most consistent current density images since the deviations of the integrals of $\vec{J}$ in each row is small.

\subsubsection{Results for Experimental Model 3}

The results obtained from experimental model 3 are presented in this subsection. The experimental model 3 and its MR magnitude image were given in Figure 4.9. The $\mathrm{z}$ component of magnetic flux density for horizontal and vertical current injection patterns are shown in Figure 5.21. 


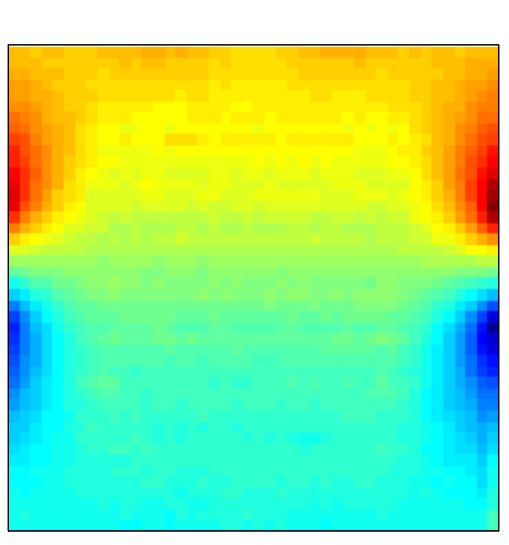

(a)
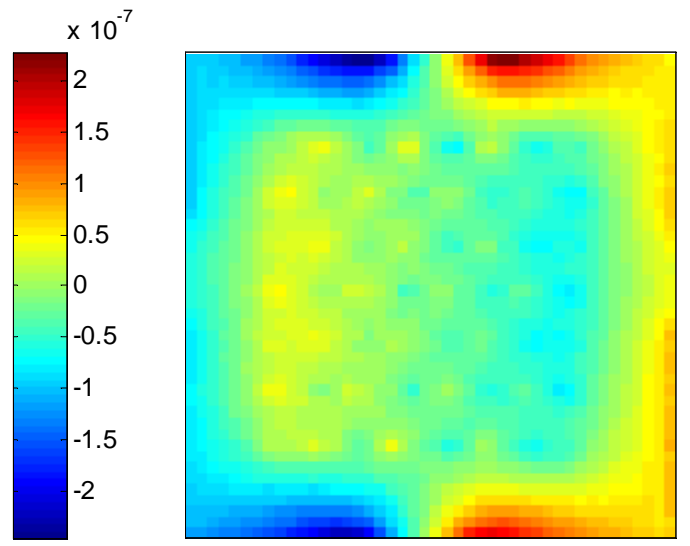

(b)

Figure 5.21 Magnetic flux density in z direction, $B_{z}(\mathrm{~T})$, a) For horizontal current injection pattern, b) For vertical current injection pattern.

The results of experimental model 3 for horizontal and vertical current injection patterns are shown in Figure 5.22 and Figure 5.23, respectively. Note that, the direction of positive y-axis is taken as negative in $J_{y}$ for vertical current injection to make the images consistent with $J_{x}$ images for horizontal current injection pattern.

When reconstruction performances of the algorithms are compared by evaluating the reconstructed current density images perceptually, it is seen that the classical MRCDI algorithm has the best performance. As it was stated, the aim of this model is to create an object that forces the current to flow between holes in vertical current injection pattern. In Figure 5.23b and Figure 5.23d, these current paths can be distinguished easily. In Figure 5.23f, the current paths are still visible but the low pass filter reduces the resolution. For horizontal current injection pattern, the reconstruction performances of the algorithms are not distinctive as in vertical current injection pattern, since the current is forced to flow in a less complex structure in this pattern.

Calculated standard deviations are listed in Table 5.6. In this table, $\sigma_{1}$ is the standard deviation of the classical MRCDI algorithm which uses three components of $\vec{B}, \sigma_{2}$ is the standard deviation of the proposed algorithm and $\sigma_{3}$ is the standard deviation of the iterative FT-MRCDI algorithm. 


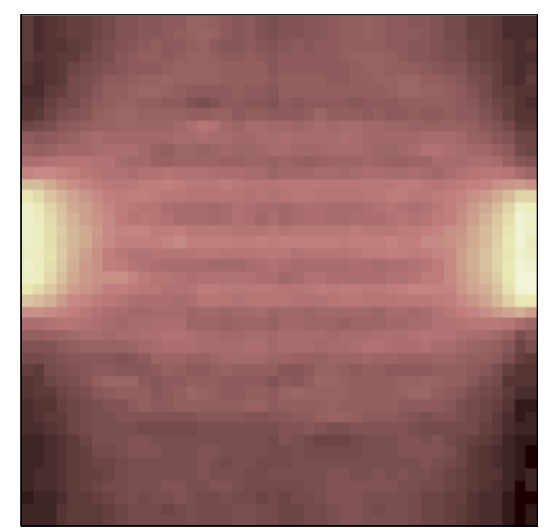

(a)

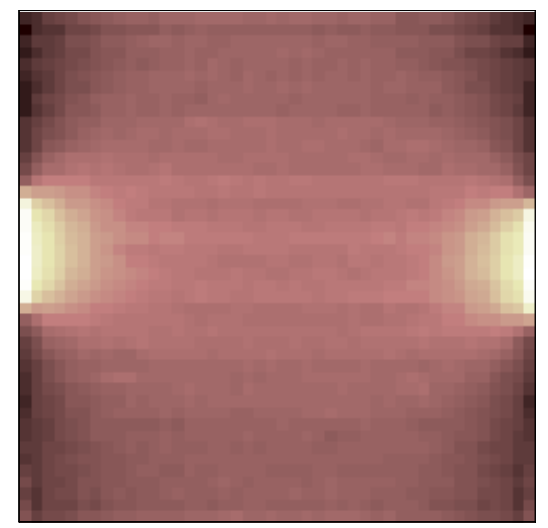

(c)

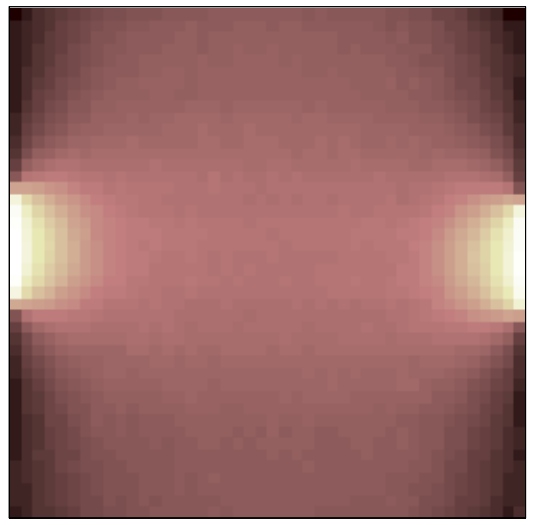

(e)
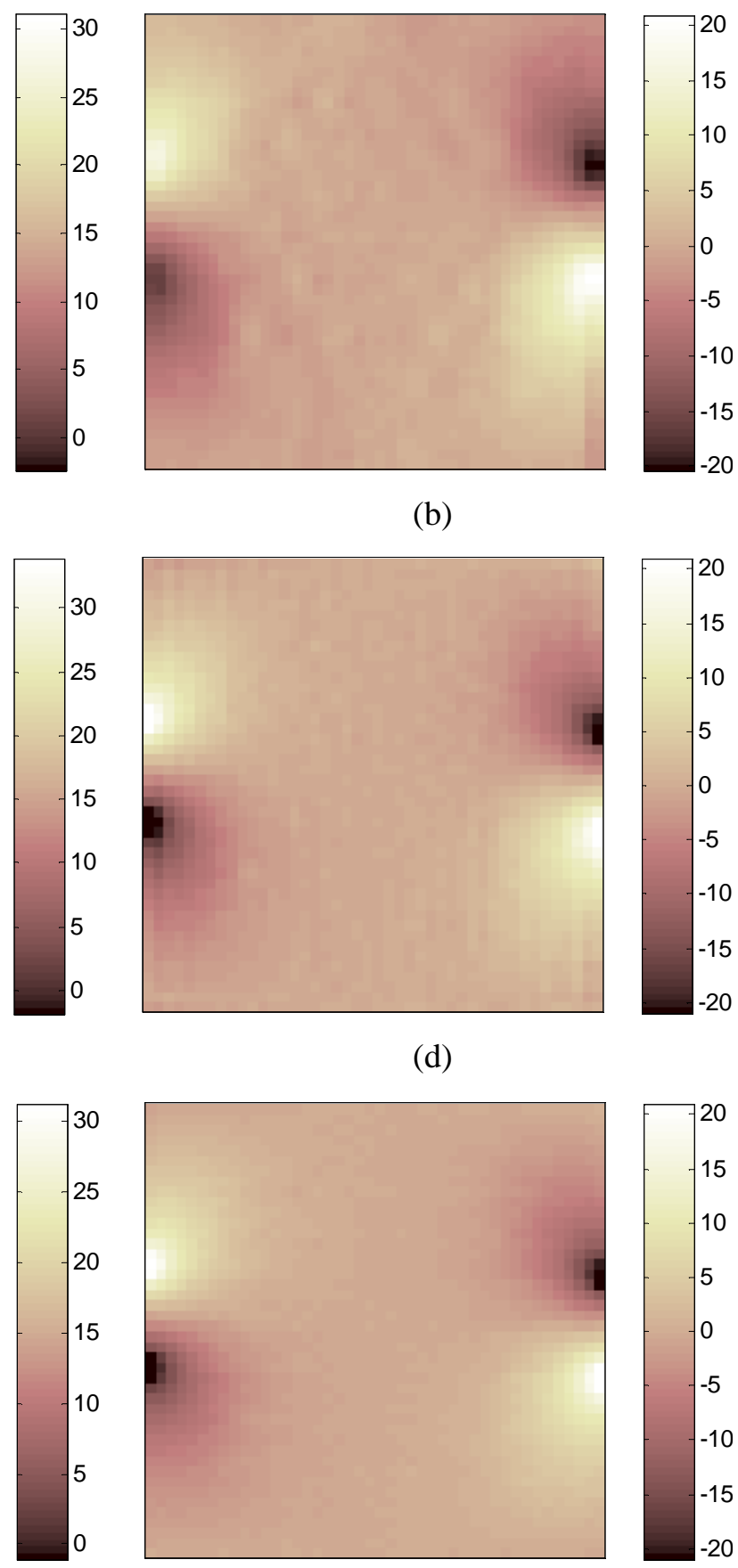

(f)

Figure 5.22 Results for horizontal current injection pattern, a) Reconstructed $J_{x}$ $\left(\mathrm{A} / \mathrm{m}^{2}\right)$ using three components of $\vec{B}$, b) Reconstructed $J_{y}\left(\mathrm{~A} / \mathrm{m}^{2}\right)$ using three components of $\vec{B}$, c) Reconstructed $J_{x}\left(\mathrm{~A} / \mathrm{m}^{2}\right)$ using the proposed algorithm, d) Reconstructed $J_{y}\left(\mathrm{~A} / \mathrm{m}^{2}\right)$ using the proposed algorithm, e) Reconstructed $J_{x}\left(\mathrm{~A} / \mathrm{m}^{2}\right)$ using Iterative FT-MRCDI algorithm, f) Reconstructed $J_{y}\left(\mathrm{~A} / \mathrm{m}^{2}\right)$ using Iterative FTMRCDI algorithm. 


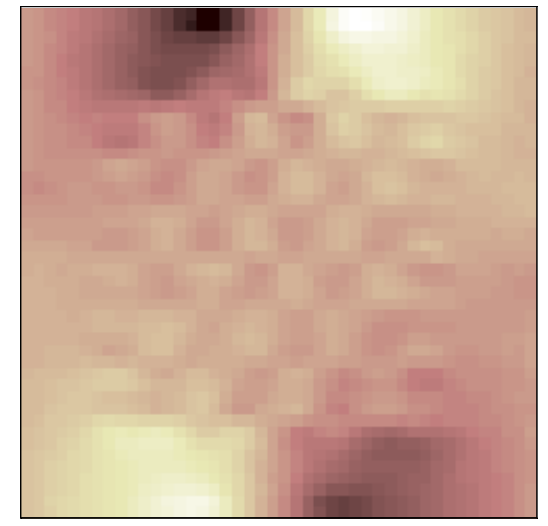

(a)

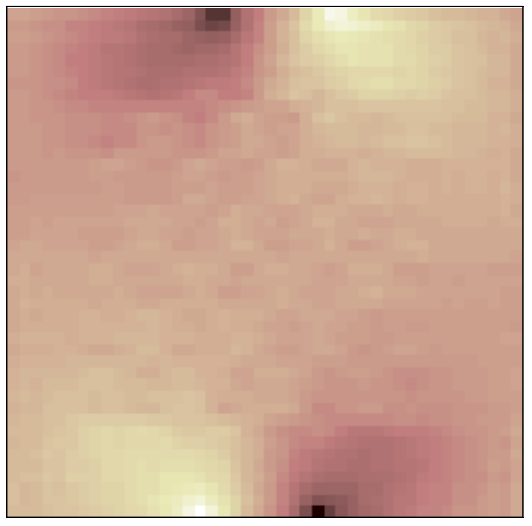

(c)

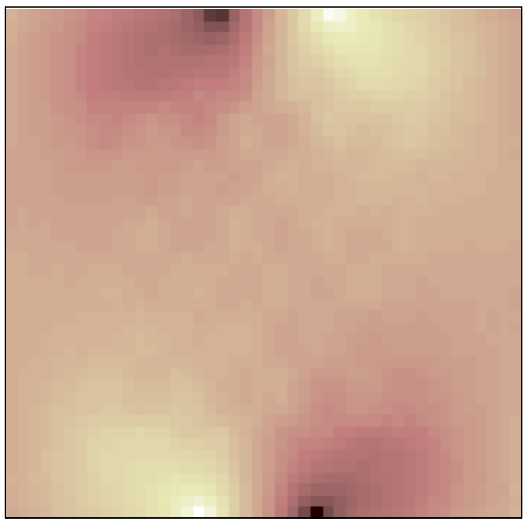

(e)
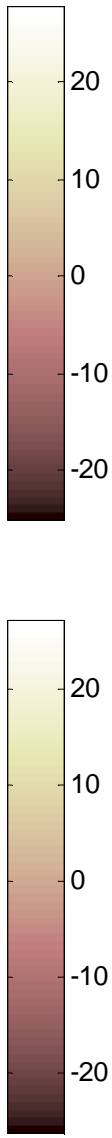

0

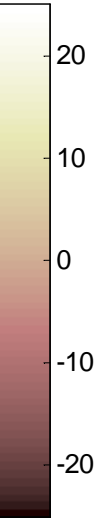

20
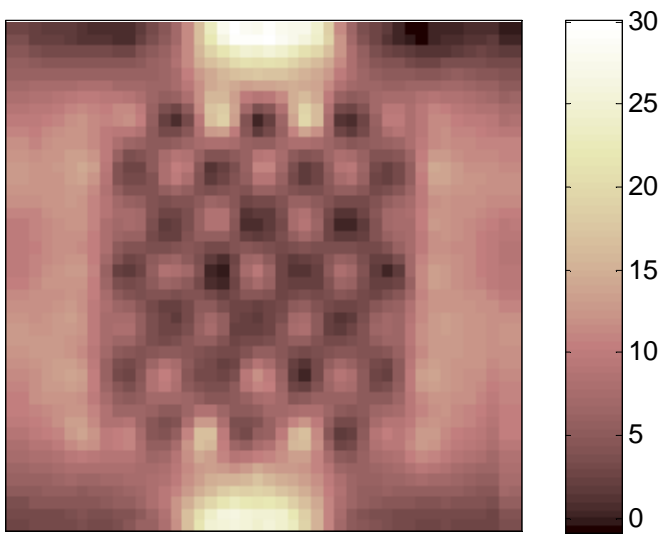

(b)

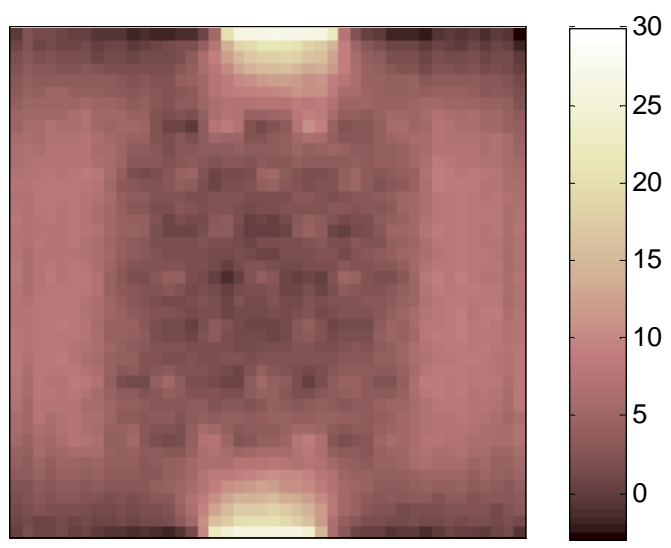

(d)

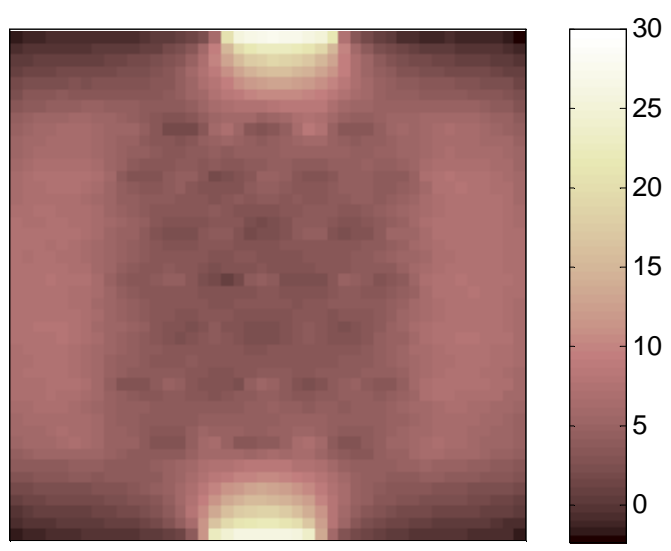

(f)

Figure 5.23 Results for vertical current injection pattern, (note that direction of positive $y$-axis is taken as negative) a) Reconstructed $J_{x}\left(\mathrm{~A} / \mathrm{m}^{2}\right)$ using three components of $\vec{B}, \mathrm{~b})$ Reconstructed $J_{y}\left(\mathrm{~A} / \mathrm{m}^{2}\right)$ using three components of $\vec{B}$, c) Reconstructed $J_{x}\left(\mathrm{~A} / \mathrm{m}^{2}\right)$ using the proposed algorithm, d) Reconstructed $J_{y}\left(\mathrm{~A} / \mathrm{m}^{2}\right)$ using the proposed algorithm, e) Reconstructed $J_{x}\left(\mathrm{~A} / \mathrm{m}^{2}\right)$ using Iterative FT-MRCDI algorithm, f) Reconstructed $J_{y}\left(\mathrm{~A} / \mathrm{m}^{2}\right)$ using Iterative FT-MRCDI algorithm. 
Table 5.6 Calculated standard deviations for experimental model 3.

\begin{tabular}{|c|c|c|}
\hline & $J_{x}$ & $J_{y}$ \\
(horizontal current injection) & (vertical current injection) \\
\hline$\sigma_{1}$ & $6.05 \times 10^{-4}$ & $15 \times 10^{-4}$ \\
\hline$\sigma_{2}$ & $2.53 \times 10^{-4}$ & $7.65 \times 10^{-4}$ \\
\hline$\sigma_{3}$ & $7.69 \times 10^{-4}$ & $4.64 \times 10^{-4}$ \\
\hline
\end{tabular}

The proposed algorithm reconstructs $J_{x}$ with smallest standard deviation in horizontal current injection pattern, whereas iterative FT-MRCDI algorithm reconstructs $J_{y}$ with the smallest standard deviation in vertical current injection pattern. 


\section{CHAPTER 6}

\section{CONCLUSIONS AND FUTURE WORK}

\subsection{Conclusions}

In this thesis, 2D MRCDI inverse problem, which relates magnetic flux density to current density, is analyzed in detail. The relation between $B_{z}, J_{x}, J_{y}$ and $\sigma$ is obtained analytically and the sensitivity of $B_{z}, J_{x}, J_{y}$ to the changes in both conductivity and the radius of the concentric inhomogeneity is analyzed. New sensitivity terms, relating the changes of $B_{z}$ to the changes in current density, are defined. Important results are obtained from these sensitivity plots. First of all, the sensitivity of $B_{z}$ to the changes in the component of current density, which is orthogonal to the current injection pattern, is plotted against the radius of the inhomogeneity placed at the center of the conductor region. From these plots it can be concluded that the sensitivity of $B_{z}$ to the changes in the orthogonal component of current density is nearly independent from the changes in the conductivity of the inhomogeneity. However, the sensitivity of $B_{z}$ to the changes in the parallel component of current density is dependent to the changes in conductivity and becomes apparent when the radius of the inhomogeneity is bigger than half of the conductor region. This situation can be explained with the fact that the change of orthogonal component of current density distribution from its value for uniform conductivity always increases as the radius of the inhomogeneity increases. This increase occurs without any dependency on the conductivity of the inhomogeneity. However, the change in parallel current density component begins to reduce when the inhomogeneity is more conductive than background and the radius of the inhomogeneity exceeds half of the size of the inhomogeneity. When the inhomogeneity is more insulating than the 
background, the change in parallel current density component always increases.

The change of $B_{z}$ from its value for uniform conductivity with respect to the changes in conductivity of the inhomogeneity also gives valuable information about the distinguishability of the inhomogeneity. From these plots, it can be concluded that the distinguishability of an object placed on the middle of the conductor region depends on its conductivity and size. If the measurement precision of a MRI system is known, then the distinguishable objects in MRI can be determined before conducting the experiment.

In this study, a novel 2D MRCDI reconstruction algorithm is proposed in addition to analytical studies. The performance of the proposed algorithm is tested with three simulation models and three experimental models. It is also compared with the iterative FT-MRCDI algorithm. Both algorithms are compared with the classical MRCDI algorithm using three components of $\vec{B}$ in experimental models.

The aim of first simulation model is to see the effect of large electrodes. The obtained results show that reconstruction performances of the algorithms are similar when the reconstructed current density images are evaluated perceptually. However, the proposed algorithm reconstructs current density with less error. The error in the reconstructed current density reaches up to $23.00 \%$ for the proposed algorithm whereas the iterative FT-MRCDI algorithm reconstructs with a maximum error of $34.18 \%$ in SNR 13 case.

Simulation model 2 contains two objects: a circular object having a conductivity of 2 $\mathrm{S} / \mathrm{m}$ and a square object prepared as a pure insulator. The aim of this model is to see the current density distribution when both insulating and conductive objects exist. The error in the reconstructed current density reaches up to $4.98 \%$ for the proposed algorithm and $20.00 \%$ for the iterative FT-MRCDI algorithm in SNR 13 case.

In simulation model 3 , it is seen that both algorithms show similar reconstruction performances for this model. It is important to note that in the iterative FT-MRCDI algorithm, the reconstructed images have lower resolutions due to the low pass filter and this filtering effect is more significant on the edges of the object. The error in reconstructed current density reaches up to $21.37 \%$ and $37.45 \%$ for the proposed 
algorithm and the iterative FT-MRCDI algorithm, respectively.

When results of the first and second simulation model are compared with results of the third simulation models, it can be seen that the error in the reconstructed current density is significantly smaller in the first and second model since in these two models conductivity transition between the background and the object is smoothened. Therefore, discretization errors are lowered providing better reconstructed current density images.

In simulation models, it can be seen that quality of the reconstructed current density images obtained by using the proposed algorithm is higher than those obtained by using the iterative FT-MRCDI algorithm. This is due to the fact that the iterative FTMRCDI algorithm requires the magnetic flux density outside the conductor region and calculates it iteratively before solving the current density. Errors in calculated magnetic flux density outside the conductor region also cause errors in the reconstructed current density. However, measured magnetic flux density inside the conductor region is sufficient for the proposed algorithm. Therefore, error in reconstructed current density images is smaller in the proposed algorithm. In the iterative FT-MRCDI algorithm, error in the calculation of magnetic flux density dominates the error due to noise. This is seen in the error tables as the overall error does not change significantly with SNR.

The experimental models are the physical realizations of the simulation models. When the results of the experimental models are evaluated together, it can be concluded that the classical MRCDI algorithm reconstructs the best images perceptually. However, there are no big differences in reconstructed image quality between the classical MRCDI algorithm and other algorithms. In fact, the proposed algorithm and the iterative FT-MRCDI algorithm can be chosen when the advantages of these algorithms are considered.

The first advantage of the algorithms using one component of $\vec{B}$ is that the elapsed time in experiments for these algorithms is one-third of the elapsed time for the classical MRCDI algorithm. Secondly, the phantom rotation, which is required in classical MRCDI algorithm, causes artifacts in the reconstructed $\vec{J}$ due to variations 
in the internal geometry during rotation. Hence, $\vec{J}$ obtained by using three components of $\vec{B}$ deviates from the true current density.

From the experimental models, it can be also concluded that the proposed algorithm is more successful when the geometry of the model is complex. For instance, in experimental model 3, resolution of the reconstructed image should be high in order to image the current paths properly. In the iterative FT-MRCDI algorithm, these paths can't be distinguished. But, these current paths are more visible in the proposed algorithm.

In the proposed algorithm and the iterative FT-MRCDI algorithm, magnetic flux

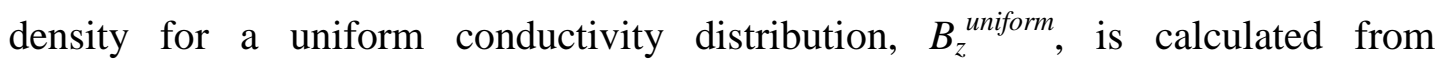
simulation. Since, subtraction of $B_{z}^{\text {uniform }}$ from $B_{z}^{\text {measured }}$ is required, misaligned electrode locations may introduce undesirable deformations in reconstructed image in experimental studies. This is a disadvantage of the algorithms. By using low pass filters, the deformations in the reconstructed current density distribution can be suppressed at the expense of reduced resolution.

In the simulation models, a noise model is prepared in order to evaluate the performance of the algorithms on noisy data and random Gaussian noise model is used for this purpose [32]. This noise model only depends on signal-to-noise ratio (SNR) of imaging system where magnetic flux density is measured. Two noise levels, SNR 30 and SNR 13, are used in simulations since SNR 30 corresponds to a MRI system with $2 \mathrm{~T}$ magnet and SNR value of $0.15 \mathrm{~T}$ METU-EE MRI system is around 13 [33]. However, the results obtained in SNR 13 case in simulations and the results obtained from experimental studies show inconsistencies. In other words, noise model in simulations doesn't reflect the case in real applications. The resistance of the receiver coil and magnetic losses caused by the sample conductivity are taken into account as the dominant sources of noise while developing noise model and this noise is independent from the strength of the acquired signal. However, the strength of the acquired signal greatly influences the quality of measured magnetic flux density in real applications. Therefore, SNR 13 noise level doesn't represent the noise level in 0.15T METU-EE MRI system exactly. 
The reconstruction time is another important criterion in the comparison of algorithms. Since the matrices used in the proposed algorithm only depend on the geometry of the subject and number of finite elements, these matrices can be calculated and stored for a subject with known geometry. Therefore, performing the matrix inversion gives the current density. The elapsed time for matrix inversion is in the order of seconds. The reconstruction time of the iterative FT-MRCDI algorithm is longer since the current density is reconstructed after iterations.

In 1988, M. Joy et al developed the MRCDI technique and conducted the first MRCDI experiment in 1989. In those days, the biggest limitations of MRCDI algorithm against the clinical applicability were the requirement for subject rotation and the amount of injected current. The requirement for subject rotation has been eliminated as reconstruction algorithms using only one component of magnetic flux density has been developed. In this thesis study, the satisfactory results of the proposed algorithm have proved once again that there is almost no need for subject rotation in a MRCDI experiment. However, the amount of injected current hasn't been reduced below the safety limits yet and remains as a strong limitation. Therefore, the topic of future studies must be reducing the amount of injected current in MRCDI experiments.

\subsection{Future Work}

In this thesis, the inverse problem of Magnetic Resonance Current Density Imaging (MRCDI), which relates magnetic flux density to current density, is investigated in detail. A novel current density reconstruction algorithm is proposed and its performance is tested with both simulated and experimental data. Some of the possible future works can be:

- The experiments can be repeated in an MRI System with higher SNR level,

- In order to use the developed algorithms on clinical applications, studies for reducing the injected current level should be made.

- Methods for evaluating the performance of the MRCDI reconstruction 
algorithms on experimental data can be developed.

- More realistic simulation noise model can be prepared. 


\section{REFERENCES}

[1] K. Boone, D. Barber, and B. Brown, "Imaging with electricity: Report of the European concerted action on impedance tomography," Journal of Medical Engineering and Technology, vol. 21, no. 6, pp. 201-232, 1997.

[2] B. M. Eyüboğlu, "Electrical Impedance Imaging: Injected Current Electrical Impedance Imaging," Wiley Encyclopedia of Biomedical Engineering, vol. 2, pp. 1195-1205, 2006.

[3] M. Philippson, "Les lois de la resitance electrique des tissus vivants," Bull Acad R Belgique Clinic Sci, vol. 7, pp. 387-403, 1921.

[4] R. P. Henderson and J. G. Webster, "An impedance camera for spatially specific measurements of the thorax," IEEE Transactions on Biomedical Engineering, vol. BME-25, no. 3, pp. 250-254, 1978.

[5] D. C. Barber, B. H. Brown, and I. L. Freeston, "Imaging spatial distributions of resistivity using applied potential tomography," Electronics Letters, vol. 19, pp. 93-95, 1983.

[6] B. H. Brown, "Electrical Impedance Tomography (EIT): a review," Journal of Medical Engineering \& Technology, vol. 27, no. 3, pp. 97-108, 2003.

[7] B. H. Brown and A. D. Seagar, "The Sheffield data collection system," Clin. Phys. Physiol. Meas., vol. 8, pp. 91-97, 1987. 
[8] W. R. Purvis, R. C. Tozer, and I. L. Freeston, "Impedance imaging using induced currents," in Annual International Conference of the Engineering in Medicine and Biology Society, vol. 12, p. 114, 1990.

[9] N. G. Gençer, M. Kuzuoğlu, and Y. Z. İder, "Electrical impedance tomography using induced currents," IEEE Transactions on Medical Imaging, vol. 13, pp. 338-350, 1994.

[10] N. G. Gençer and N. M. Tek, "Electrical conductivity imaging via contactless measurements," IEEE Transaction on Medical Imaging, vol. 18, no. 7, pp. 617$627,1999$.

[11] B. M. Eyüboğlu, "Magnetic Resonance Electrical Impedance Tomography," Wiley Encyclopedia of Biomedical Engineering, vol. 4, pp. 2154-2162, 2006.

[12] M. Joy, G. Scott, and M. Henkelman, "In vivo detection of applied electric currents by mangetic resonance imaging," Magnetic Resonance Imaging, vol. 7, pp. 89-94, 1989.

[13] B. M. Eyüboğlu, "Magnetic Resonance Current Density Imaging," Wiley Encyclopedia of Biomedical Engineering, vol. 4, pp. 2147-2153, 2006.

[14] N. Zhang, "Electrical impedance tomography based on current density imaging," University of Toronto, Dept. of Electrical and Electronics Eng., Toronto, Canada, Master Thesis 1992.

[15] Y. Z. İder, S. Onart, and W. R. Lionheart, "Uniqueness and Reconstruction in Magnetic Resonance Electrical Impedance Tomography (MR-EIT)," Physiological Measurement, vol. 24, pp. 591-594, 2003.

[16] M. S. Özdemir, B. M. Eyüboğlu, and O. Özbek, "Equipotential projection-based 
magnetic resonance electrical impedance tomography and experimental realization," Physics In Medicine and Biology, vol. 49, pp. 4765-4783, 2004.

[17] O. Kwon, E. J. Woo, J. R. Yoon, and J. K. Seo, "Magnetic Resonance Electrical Impedance Tomography (MREIT): Simulation Study of J-Substitution Algorithm," IEEE Transactions on Biomedical Engineering, vol. 49, no. 2, pp. 160-167, 2002.

[18] R. Boyacıoğlu, "Performance evaluation of current density based magnetic resonance electrical impedance tomography reconstruction algorithms," Middle East Technical University, Ankara, MSc Thesis 2009.

[19] J. K. Seo, J. R. Yoon, E. J. Woo, and O. Kwon, "Reconstruction of conductivity and current density images using only one component of magnetic field measurements," IEEE Transactions on Biomedical Engineering, vol. 50, pp. 1121-1124, 2003.

[20] C. Park, E. J. Park, E. J. Woo, O. Kwon, and J. K. Seo, "Static conductivity imaging using variational gradient $\mathrm{Bz}$ algorithm in magnetic resonance electrical impedance tomography," Physiological Measurement, vol. 25, pp. 257-269, 2004.

[21] Y. Z. Ider and Ö. Birgül, "Use of magnetic field generated by the internal distribution of injected currents for electrical impedance tomography (MREIT)," Electric Turkish J. of Elec. Eng and Comp. Sci., vol. 6, no. 3, pp. 215225, 1998.

[22] Gökhan Eker, "Performance evaluation of magnetic flux density based magnetic resonance electrical impedance tomography reconstruction algorithms," Middle East Technical University, Ankara, MSc Thesis 2009. 
[23] Bradley J. Roth, Nestor G. Sepulveda, and John P. Wikswo, "Using a magnetometer to image a two-dimensional current distribution," J. Appl. Phys., vol. 65 , no. 1, pp. 361-372, 1989.

[24] Predrag Pesikan, Michael L. G. Joy, Greig C. Scott, and R. Mark Henkelman, "Two-dimensional current density imaging," IEEE Transactions on Instrumentation and Measurement, vol. 39, no. 6, pp. 1048-1053, 1990.

[25] Y. Ziya Ider, "Bz-substitution MR-EIT and Fourier Transform MR-CDI: Two new algorithms," in World Congress on Medical Physics and Biomedical Engineering, Seoul, Korea, 2006, pp. 3803-3806.

[26] G. C. Scott, M. L. G. Joy, R. L. Armstrong, and R. M. Henkelman, "Measurement of nonuniform current density by magnetic resonance," IEEE Transactions on Medical Imaging, vol. 10, no. 3, pp. 362-374, 1991.

[27] Ö. Birgül, "Development of reconstruction algorithms for magnetic resonance electrical impedance tomography and experimental realization," Middle East Technical University, Ankara, PhD Thesis 2002.

[28] O. Özbek, "Imaging current density distribution using 0.15 T magnetic resonace tomography," Middle East Technical University, Ankara, MSc Thesis 2002.

[29] E. Değirmenci, "High resolution imaging of anistropic conductivity with magnetic resonance-electrical impedance tomography (MR-EIT)," Middle East Technical University, Ankara, PhD Thesis 2010.

[30] M. Özsüt, "Design and implementation of labview based data acquisition and image reconstruction environment for METU-MRI System," Middle East Technical University , Ankara, MSc Thesis 2005. 
[31] Y. Z. Ider and O. Arıkan, "Iterative Fourier Transform Magnetic Resonance Current Density Imaging (FT-MRCDI)," Proc. Intl. Soc. Mag. Reson. Med., vol. 15, p. 1778, 2007.

[32] G. C. Scott, M. L. Joy, R. L. Armstrong, and R. M. Henkelman, "Sensitivity of magnetic resonance current density imaging," Journal of magnetic resonance, vol. 97, no. 2, pp. 235-254, 1992.

[33] Ö. Birgül, B. M. Eyüboğlu, and Y. Z. Ider, "Current constrained voltage scaled reconstruction (CCVSR) algorithm for MR-EIT and its performance with different probing current patterns," Phys. Med. Biol., vol. 48, pp. 653-671, 2003. 


\section{APPENDIX A}

\section{COMPUTATION OF CIRCULAR HARMONIC COEFFICIENTS}

In Appendix A, the calculation of circular harmonic coefficients will be explained in detail. In Chapter 3, it was mentioned that the solution of Laplace's equation could be represented by an infinite sum of circular harmonics as

$$
\Phi(r, \varphi)=\left\{\begin{array}{l}
\Phi_{1}(r, \varphi) \text { if } 0 \leq r \leq b \\
\Phi_{2}(r, \varphi) \text { if } b \leq r \leq a
\end{array}\right.
$$

where

$$
\begin{gathered}
\Phi_{1}(r, \varphi)=\sum_{n=1}^{\infty} r^{n}\left[A_{n} \cos (n \varphi)+B_{n} \sin (n \varphi)\right] \\
\Phi_{2}(r, \varphi)=\left\{\begin{array}{l}
\sum_{n=1}^{\infty} r^{n}\left[E_{n} \cos (n \varphi)+F_{n} \sin (n \varphi)\right] \\
+\sum_{n=1}^{\infty} r^{-n}\left[G_{n} \cos (n \varphi)+H_{n} \sin (n \varphi)\right]
\end{array}\right\}
\end{gathered}
$$

and boundary conditions of this problem was given as

$$
\begin{gathered}
\Phi_{1}(b, \varphi)=\Phi_{2}(b, \varphi) \\
-\left.\sigma_{1} \frac{\partial \Phi_{1}(r, \varphi)}{\partial r}\right|_{r=b}=-\left.\sigma_{2} \frac{\partial \Phi_{2}(r, \varphi)}{\partial r}\right|_{r=b} \\
-\left.\sigma_{2} \frac{\partial \Phi_{2}(r, \varphi)}{\partial r}\right|_{r=a}=j(\varphi)
\end{gathered}
$$


By using the boundary condition in Equation (A.4) and reorganizing the terms, the following equation is obtained:

$$
\begin{aligned}
& A_{n}=E_{n}+b^{-2 n} G_{n} \\
& B_{n}=F_{n}+b^{-2 n} H_{n}
\end{aligned}
$$

Another equation can be obtained from the boundary condition in Equation (A.5) as

$$
\begin{aligned}
& \sigma_{1} A_{n}=\sigma_{2} E_{n}-\sigma_{2} b^{-2 n} G_{n} \\
& \sigma_{1} B_{n}=\sigma_{2} F_{n}-\sigma_{2} b^{-2 n} H_{n}
\end{aligned}
$$

Finally, by using Equation (A.6), the following equation is obtained:

$$
\begin{aligned}
& E_{n}-a^{-2 n} G_{n}=\frac{-C_{n}}{\sigma_{2} n a^{n-1}} \\
& F_{n}-a^{-2 n} H_{n}=\frac{-S_{n}}{\sigma_{2} n a^{n-1}}
\end{aligned}
$$

Then, by solving Equations (A.7) and (A.8), $E_{n}$ and $F_{n}$ are obtained as:

$$
\begin{aligned}
& E_{n}=A_{n}\left(\frac{\sigma_{1}+\sigma_{2}}{2 \sigma_{2}}\right) \\
& F_{n}=B_{n}\left(\frac{\sigma_{1}+\sigma_{2}}{2 \sigma_{2}}\right)
\end{aligned}
$$

Substituting Equation (A.10) in Equation (A.7) gives

$$
\begin{aligned}
& A_{n}\left(1-\frac{\sigma_{1}+\sigma_{2}}{2 \sigma_{2}}\right)=b^{-2 n} G_{n} \\
& B_{n}\left(1-\frac{\sigma_{1}+\sigma_{2}}{2 \sigma_{2}}\right)=b^{-2 n} H_{n}
\end{aligned}
$$

Finally, by substituting Equation (A.10) and Equation (A.11) in Equation (A.9), the coefficients of circular harmonics are calculated as

$$
A_{n}=\frac{-2 C_{n} b^{-2 n}}{n a^{n-1}\left[\left(\sigma_{1}+\sigma_{2}\right) b^{-2 n}-\left(\sigma_{2}-\sigma_{1}\right) a^{-2 n}\right]}
$$




$$
\begin{gathered}
B_{n}=\frac{-2 S_{n} b^{-2 n}}{n a^{n-1}\left[\left(\sigma_{1}+\sigma_{2}\right) b^{-2 n}-\left(\sigma_{2}-\sigma_{1}\right) a^{-2 n}\right]} \\
E_{n}=\frac{-\left(\sigma_{1}+\sigma_{2}\right) C_{n} b^{-2 n}}{n a^{n-1}\left[\left(\sigma_{1}+\sigma_{2}\right) b^{-2 n}-\left(\sigma_{2}-\sigma_{1}\right) a^{-2 n}\right] \sigma_{2}} \\
F_{n}=\frac{-\left(\sigma_{1}+\sigma_{2}\right) S_{n} b^{-2 n}}{n a^{n-1}\left[\left(\sigma_{1}+\sigma_{2}\right) b^{-2 n}-\left(\sigma_{2}-\sigma_{1}\right) a^{-2 n}\right] \sigma_{2}} \\
G_{n}=\frac{-\left(\sigma_{2}-\sigma_{1}\right) C_{n}}{n a^{n-1}\left[\left(\sigma_{1}+\sigma_{2}\right) b^{-2 n}-\left(\sigma_{2}-\sigma_{1}\right) a^{-2 n}\right] \sigma_{2}} \\
H_{n}=\frac{-\left(\sigma_{2}-\sigma_{1}\right) S_{n}}{n a^{n-1}\left[\left(\sigma_{1}+\sigma_{2}\right) b^{-2 n}-\left(\sigma_{2}-\sigma_{1}\right) a^{-2 n}\right] \sigma_{2}}
\end{gathered}
$$




\section{PUBLICATIONS}

\section{International Conferences}

- A. Ersöz and B. M. Eyüboğlu, "Magnetic Resonance Current Density Imaging Using One Component of Magnetic Flux Density: An Experimental Study," XII Mediterranean Conference on Medical and Biological Engineering and Computing 2010, Chalkidiki-Greece, IFMBE Proceedings vol. 29/II, pp. 248-251, 2010.

\section{National Conferences}

- A. Ersöz and B. M. Eyüboğlu, "Magnetic Resonance Current Density Imaging Using One Component of Magnetic Flux Density," 15th National Biomedical Engineering Meeting (BIYOMUT), Antalya-Turkey, 2010. 